\title{
A network information theoretic framework to characterise muscle synergies in space and time
}

\author{
David Ó’ Reilly; Ioannis Delis ${ }^{1}$ \\ ${ }^{1}$ Faculty of Biological sciences, School of Biomedical sciences, University of Leeds, Leeds, United Kingdom.
}

Abstract

Coordinated movement is thought to be simplified by the nervous system through the activation of muscle synergies. Current approaches to muscle synergy extraction rely on dimensionality reduction algorithms that impose limiting constraints. To capture large-scale interactions between muscle activations, a more generalised approach that considers the complexity and nonlinearity of the motor system is required. Here we developed a novel framework for muscle synergy extraction that relaxes model assumptions by using a combination of informationand network theory and dimensionality reduction. This novel framework can capture spatial, temporal and spatiotemporal interactions, producing distinct spatial groupings and both tonic and phasic temporal patterns. Furthermore, our framework identifies submodular structures in the extracted synergies that exemplify the fractal modularity of the human motor system. To demonstrate the versatility of the methodology, we applied it to two benchmark datasets of arm and whole-body reaching movements. Readily interpretable muscle synergies spanning multiple spatial and temporal scales were identified that demonstrated significant task dependence, ability to capture trial-to-trial fluctuations, a scale-invariance with dataset complexity and a substantial concordance across participants. Finally, we position this framework as a bridge between existing models and recent network-theoretic endeavours by discussing the continuity and novelty of the presented findings. 
bioRxiv preprint doi: https://doi.org/10.1101/2021.10.15.464450; this version posted October 20, 2021. The copyright holder for this preprint (which was not certified by peer review) is the author/funder, who has granted bioRxiv a license to display the preprint in perpetuity. It is made available under aCC-BY-NC 4.0 International license.

\section{Introduction}

The question of whether the control of movement can be characterised by a simplified, low-dimensional strategy is a pertinent one in the motor control literature [1-4]. When one considers the numerous degrees-offreedom available to the human body at the neural, musculoskeletal and dynamic level for a given movement, the task of selecting an adequate strategy from the set of redundant solutions becomes a computationally intensive operation [3]. Along with this, the capacity for the human body to adapt pre-existing movements and allow for the emergence of novel strategies calls into question, within the context of biomechanical constraints and environmental demands, the nature of the neural constraints on movement $[5,6]$. It has been hypothesized that the central nervous system (CNS) harbours a modular architecture at the level of the spine that activates motor primitives and through the combination of these building-blocks, complex motor patterns can be efficiently performed $[3,4]$. This hypothesis allows for the control of groups of muscles ('muscle synergies') rather than the more computationally intensive, individual control of each degree-of-freedom in the brain. Evidence for this phenomenon comes most conclusively from animal studies but a significant degree of indirect evidence is also accumulating in the human population $[7,8]$, during development and with training experience for example [9].

The recent efforts in the motor control literature provide a foundation for furthering our understanding of the mechanisms underlying modularity in human motor control. Among those efforts, different research groups have formulated mathematical definitions to factorize EMG signals using unsupervised machine-learning in the spatial [11], temporal [12] and spatiotemporal domains [13] or their unification through the space-by-time model [14]. These investigations produced novel insights such as the presence of both task-specific and -shared synergies and the complexity of synergies being linearly related to neurological impairment $[15,16]$. These muscle synergy models are typically implemented using non-negative matrix factorisation (NMF) but tensor decompositions have also recently been utilised with the particular advantage of concurrent extraction of both spatial and temporal synergies and their task-dependent modulations $[14,17]$. Nonetheless, NMF and its higher-dimensional variants are constrained to extract linear representations of the EMG activity and so may not fully capture the non-linear characteristics of the musculoskeletal system. Furthermore, the incorporation of task space variables in synergy extraction using the described models ('functional synergies') has revealed consistent relationships between muscle activity and task performance, with unique biomechanical functions exemplified by the identified synergies $[18,19]$. In contrast to the muscle space, task parameters may be negative and have mixing coefficients that don't necessarily correspond with EMG data [10]. The current muscle synergy models don't facilitate the incorporation of signals with such diverse properties and therefore their extension to include task parameters violates underlying model assumptions. A more generalised and non-parametric formulation of the current muscle synergy models may accommodate these limitations. Moreover, a generalised approach will also be more amenable to future avenues of research on movement modularity discussed at length by [1], including the falsifiability of muscle synergy patterns by neural signals derived elsewhere serving as model constraints. Thus, the development of an appropriate methodological approach that can meet these requirements is incentivised.

In the current study, we sought to develop a novel framework for the characterisation of muscle synergies using a combination of information- and network-theory and dimensionality reduction. Both information- and network theory have proven useful in the analysis of such muscle couplings with novel insights gained in both the temporal and frequency domains and by elucidating the shaping of these couplings by anatomical constraints for example [20-22]. Our proposed framework here extends existing muscle synergy models by exploiting the advantageous properties of a novel mutual information formulation for the efficient extraction of salient features across space, time, repetitions and experimental conditions. From the outset, we sought to stringently align the attributes of this framework with the unique characteristics of the synergy concept while positioning it as a useful tool for the progression of the motor control research field. A novel model selection procedure is introduced, where the extracted informational dynamics are modelled as a multiplex network and the predominant clusters identified. The number of clusters identified then serves as the empirical input parameter for dimensionality reduction. Following the presentation of this framework, we applied it to two benchmark datasets of point-to-point reaching movements. We identified functionally and physiologically meaningful synergies that demonstrate a high level of consistency in structure, noise correlations and task dependence across participants. A submodular structure was also highlighted across all synergies that captured functional connections that transcended biomechanical constraints and exemplified the fractal modularity of the human motor system. Finally, we discuss the continuity of these models with previous research, novel insights gained along with potential directions for future research using this generalised approach. An open-source, easy-to-use Matlab GUI (https://github.com/DelisLab/GCMI-synergy- 
extraction) is available for readers to implement this novel computational framework on their own data as described here.

\section{Materials and Methods}

\section{Informational dynamics in motor control}

A muscle synergy consists of two or more muscles acting as a functional unit during the execution of a coordinated movement and has the identifiable characteristics of a sharing pattern, reciprocal compensation and task dependence [23]. In other words, muscles may share a consistent pattern of activation in space and time, their activations are interdependent and can be adjusted when deviations are experienced, and as elemental variables they can be re-organised for different objective functions. With such complex interdependencies between components, the accurate modelling of these emergent properties during naturalistic behaviour is challenging, requiring a high degree of computational sophistication. This analytical challenge is accompanied by the presence of noisy communication channels that innervate the numerous components of the system, requiring statistical tools that are robust to noise. The Claude Shannon communication system model constitutes a relevant statistical tool for this endeavour as information theoretic measures such as mutual information (MI) have been specifically developed for the analysis of noisy communication channels and are general in their applicability [24]. Recent applications of MI to neural circuitry have been fruitful, with dependence between cortical- and spinal-level activations and motor behaviours elucidated [25-27]. In the following, we briefly present the foundational concepts incorporated in the presented framework that allow for interactions between muscles in space and time to be quantified as informational dynamics in a computationally efficient and noise robust manner.

\section{Mutual information}

The $\mathrm{MI}$ between a pair of muscles $M_{x}$ and $M_{y}\left(I\left(M_{x} ; M_{y}\right)\right)$ (or pair of timepoints $T_{x}$ and $T_{y}\left(I\left(T_{x} ; T_{y}\right)\right)$ ) can be thought of as the difference between the entropies of the individual variables $\left(H\left(M_{x}\right)+H\left(M_{y}\right)\right)$ and their joint entropy $\left(H\left(M_{x}, M_{y}\right)\right)$. The entropy of a random variable is the degree of uncertainty of getting a possible outcome from the given distribution and so MI quantifies the reduction of uncertainty in $M_{y}$ due to $M_{x}$.

$I\left(M_{x} ; M_{y}\right)=H\left(M_{x}\right)+H\left(M_{y}\right)-H\left(M_{x}, M_{y}\right)$

Equation 1.1

In doing so, $\mathrm{MI}$ captures the non-linear dependence between $M_{x}$ and $M_{y}$ in the unit known as bits. This expression of $\mathrm{MI}$ will aid in the communication of the novel $\mathrm{Ml}$ estimate in the next section. There are a number of equivalent expressions for $\mathrm{MI}$ in the literature, another is given in equation 1.2. Here, $\mathrm{Ml}$ is quantified as the difference between the entropy of $M_{x}$ and the conditional entropy of $M_{x}$ given $M_{y}, H\left(M_{x} \mid M_{y}\right)$. Using this expression, it can be seen that when $M_{y}$ completely determines $M_{x}$ (and vice-versa), the $\mathrm{Ml}$ is equal to $H\left(M_{x}\right)$.

$I\left(M_{x} ; M_{y}\right)=H\left(M_{x}\right)-H\left(M_{x} \mid M_{y}\right)$

Equation 1.2

Here we employ a parametric estimator for entropy and MI which enables a reliable estimation of information using limited samples and is computationally efficient [28]. Their formulations are presented in the closed-form in equations 1.3.1 and 1.3.2 respectively. Entropy is expressed as a function of the covariance matrix determinant $\left(\left|\sum\right|\right)$ with dimensionality $k . \sum_{M_{x}}$ and $\sum_{M_{y}}$ are the covariance matrices of two Gaussian variables (EMG channels, timepoint vectors etc.) and $\sum_{M_{x y}}$ is the covariance matrix for the joint variable contrasted against these individual covariance's within the determinant. A bias-correction term was applied to these estimations as illustrated in [29].

$$
\begin{aligned}
& H(X)=\frac{1}{2 \ln 2} \ln \left[(2 \pi e)^{k}\left|\sum\right|\right] \\
& I(X ; Y)=\frac{1}{2 \ln 2} \ln \left[\frac{\left|\sum_{M_{x}}\right|\left|\sum_{M_{y}}\right|}{\left|\sum_{M_{x y}}\right|}\right]
\end{aligned}
$$$$
\text { Equation 1.3.1 }
$$

Equation 1.3.2

\section{Gaussian copula Mutual information}

Similarly to $\mathrm{MI}$, a copula $(c)$ is a statistical measure of non-linear dependence between a pair of random variables but in the case of $c$, this estimate has two advantageous properties described in Sklar's theorem and the 
invariance theorem [29,30]. Briefly, a given multivariate cumulative distribution function (CDF) can be described by two components, the $c$ linking the variables and their marginal CDFs in such a way that when the marginal CDFs are continuous, $c$ is unique. $c$ is a probability density over the unit square that is formulated through the rank normalization of individual variables and rescaling to a range between $0-1$. In linking two random variables, $c$ is therefore directly related to $\mathrm{MI}$ (Equation 2.1) [28].

$H\left(M_{x}, M_{y}\right)=H\left(M_{x}\right)+H\left(M_{y}\right)+H(c)$

Equation 2.1

As shown in equation 2.1, the joint entropy of $M_{x}$ and $M_{y}$ is equal to their individual entropies and the entropy of $c$. Plugging equation 2.1 into equation 1.1, we see that the marginal entropies will cancel out (Equation 2.2), meaning negative $H(c)$ is equal to the $\mathrm{MI}$ between the $M_{x}$ and $M_{y}$ variables referred to hereafter as the Gaussian copula mutual information (GCMI) [28].

$I\left(M_{x} ; M_{y}\right)=-H(c)$

Equation 2.2

An attractive quality of this formulation is that the empirical copula can maintain this encapsulated relationship between variables following a monotonic transformation of their individual distributions [30]. Thus, it is appropriate to implement the computationally efficient parametric estimation in equation 1.3.1 following a marginal transformation of the individual random variables to a Gaussian distribution. Furthermore, a Gaussian distribution is desirable here as it has the maximum entropy of any form and therefore the resulting GCMI estimates serve as a conservative lower-bound on the true MI.

GCMI will form the cornerstone for the muscle synergy framework presented here, representing a robust, nonlinear statistical measure of dependence that can be applied to a broad range of calculations involving uni- and multi-variate samples and in permutation testing for example. An opensource toolbox containing the informationtheoretic measures described here was utilised in Matlab software [28]. 
bioRxiv preprint doi: https://doi.org/10.1101/2021.10.15.464450; this version posted October 20, 2021. The copyright holder for this preprint (which was not certified by peer review) is the author/funder, who has granted bioRxiv a license to display the preprint in perpetuity. It is made available under aCC-BY-NC 4.0 International license.

Information extraction from EMG activity
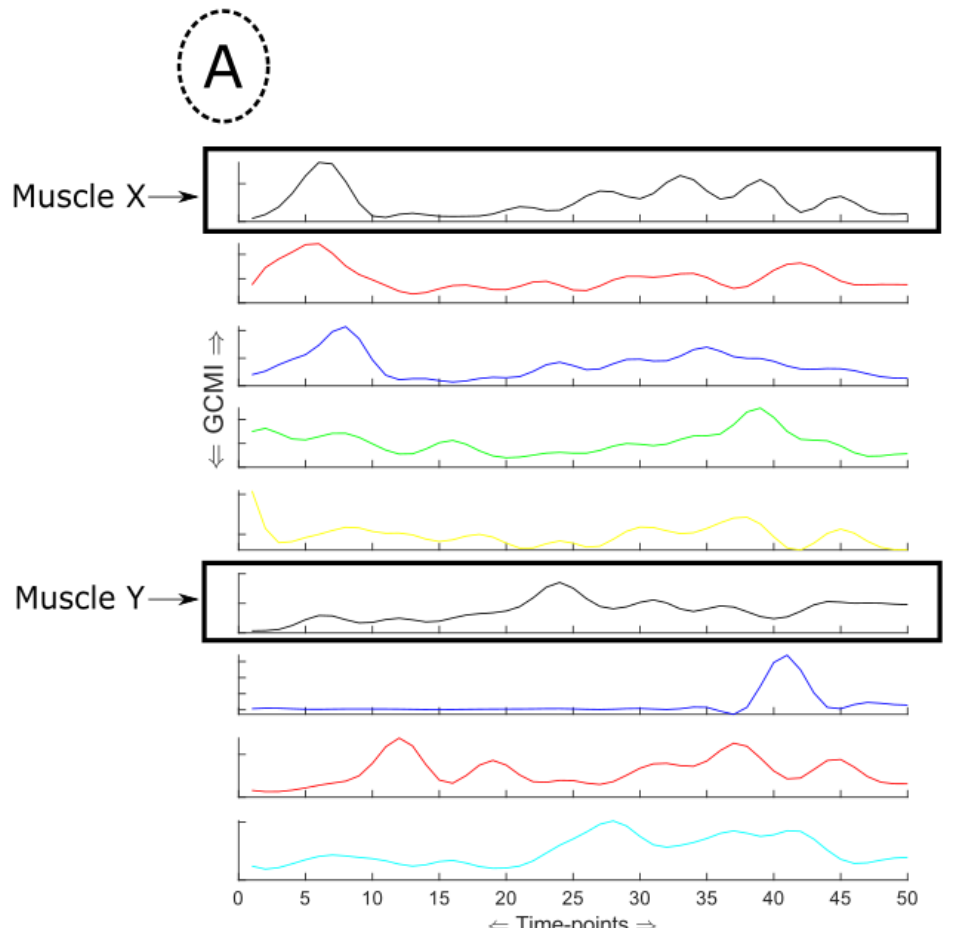<smiles></smiles>
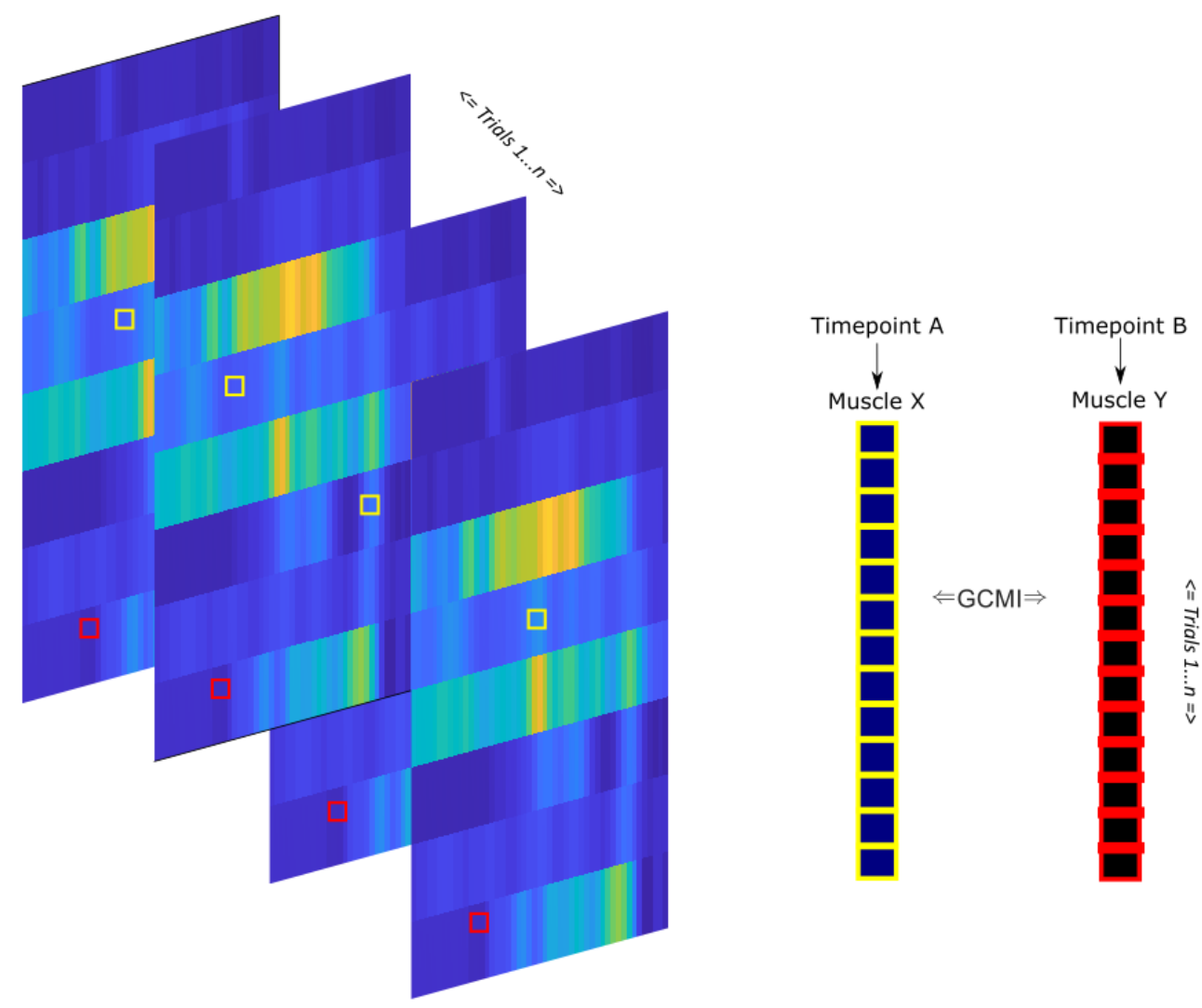

Fig 1: (A) An example of spatial information extraction with the GCMI computed from the EMG activity of two muscles across all timepoints in a single trial. (B) An example of temporal information extraction where the GCMI is computed between two time-vectors across all nine muscles in a single trial. (C) The space-time information extraction is illustrated where timepoint $\mathbf{A}$ for a single muscle across all trials is extracted, creating a vector that is used to compute the GCMI against a similar vector for another muscle at timepoint $\mathrm{B}$.

To compute couplings between muscles $M$ across time $t=1, \ldots, T$ (i.e. synergies in the spatial domain), we took a pair of muscle activations $M_{x}, M_{y}$ for a single trial $n$ and determined the GCMI between them $I_{n}\left(M_{x} ; M_{y}\right)$ (Fig.1 (A)). This procedure was iterated over each unique combination of muscles $k=1, \ldots, K$ for all available trials $\mathrm{s}=$ $1 \ldots N$, creating symmetric lower-triangular matrices equal in dimensions to the number of muscles in the dataset (Fig.2(B)). These matrices for each trial were vectorised and concatenated (Fig.2 (C)), producing a $s$ (No. of trials) $\times k=$ 
$m(m-1) / 2$ (No. of muscle pairs) matrix $\left(I_{\text {Space }}=\left[I_{s^{1}} \ldots I_{S^{n}}\right]\right)$ (Fig. 2(D)). This matrix served as input into a dimensionality reduction method known as projective non-negative matrix factorisation (PNMF), a variant of the frequently used NMF that has demonstrated a superior capacity for producing sparse representations of high dimensional data and identifying subspace clusters [31,32]. This variant acts as a hybrid of NMF and Principal Component Analysis by using singular value decomposition (SVD) to extract linear, orthogonal features that are positively-constrained. In the current implementation, the SVD was initialised using non-negative Double SVD while the model-rank (i.e. number of extracted components) was determined using a novel procedure (Fig.2(E)) described in detail in the 'Model rank selection' section that follows [33].

In the spatial domain, the factorisation of the input matrix, a matrix consisting of unique muscle pairings $m=1 \ldots . k$ across trials $s=1 \ldots . . n$ is described below in vector sum and matrix notation (Equation 3.1). Here the set of synergy weights $(V)$ consists of column vectors $(v)$ representing clusters of muscle couplings across time in the $j t h$ module. The set of activation coefficients $(A)$ consists of row vectors, $a_{j}^{s^{n}}$, are module- and trial-specific scalar values, inferred to be the neural commands driving the synergy patterns.

$$
\begin{gathered}
I_{\text {Space }} \approx \\
\approx V \\
\left(\begin{array}{ccc}
\left(m^{1}\right)^{s^{1}} & \cdots & \left(m^{1}\right)^{s^{n}} \\
\vdots & \ddots & \vdots \\
\left(m^{k}\right)^{s^{1}} & \cdots & \left(m^{k}\right)^{s^{n}}
\end{array}\right) \approx\left(\begin{array}{lll}
a_{j}^{s^{1}} & \ldots & a_{j}^{s^{n}}
\end{array}\right) \cdot\left(\begin{array}{c}
\boldsymbol{v}_{j}^{m^{1}} \\
\vdots \\
\boldsymbol{v}_{j}^{m^{k}}
\end{array}\right)+\text { residuals }
\end{gathered}
$$

Equation 3.1.

To determine the temporal dependencies across muscles, we computed GCMI between all unique pairs of timepoint vectors of length $M(\mathrm{~L}=\mathrm{t}(\mathrm{t}-1) / 2)$, creating an $L \times s$ matrix $\left(I_{\text {Time }}\right)$. We then implemented the procedure described in Fig.2(A-E), resulting in a factorised output of temporal synergy weights $(W)$ and activation coefficients $(A)$. The factorised output in the temporal domain is described in equation 3.2 where the set of temporal synergy weights consists of row vectors $(\boldsymbol{w})$ representing clusters of temporal coupling across muscles for the $i$ th module.

$$
I_{\text {Time }} \quad \text { W } \quad W
$$

$\left(\begin{array}{ccc}\left(t^{1}\right)^{s^{1}} & \cdots & \left(t^{1}\right)^{s^{n}} \\ \vdots & \ddots & \vdots \\ \left(t^{L}\right)^{s^{1}} & \cdots & \left(t^{L}\right)^{s^{n}}\end{array}\right) \approx\left(\begin{array}{lll}\boldsymbol{w}_{i}^{t^{1}} & \ldots & \boldsymbol{w}_{i}^{t^{L}}\end{array}\right) \cdot\left(\begin{array}{c}a_{i}^{s^{1}} \\ \vdots \\ a_{i}^{s^{n}}\end{array}\right)+$ residuals

Equation 3.2.

Finally, as an extension of the above muscle synergy models and of previous work [14], spatial and temporal modules were concurrently extracted in a unifying space-time model through the following computation (Fig. 1(C)). We extracted the EMG activity of a particular muscle $M_{x}$ at a particular timepoint $T_{x}$ across all trials and computed GCMI between this vector and that of a similar vector for muscle $M_{y}$ at timepoint $T_{y}$. Once again we iterated this computation over all unique combinations of these vectors and then implemented the synergy extraction procedure described in Fig.2(A-E), i.e. we vectorized the GCMI values computed between these trial-to-trial vectors and concatenated them into a $k=m(m-1) / 2$ (No. of muscle pairs) $\times \mathrm{L}=\mathrm{T}(\mathrm{T}-1) / 2$ (No. of timepoint pairs) input matrix $\left(I_{\text {Space-Time }}\right)$. This enabled us to determine trial-to-trial dependencies that are consistent across spatial $(V)$ and temporal $(W)$ dimensions. The factorised output for the space-time model is described below (Equation 3.3), with $v$ and $w$ being the spatial and temporal synergy weights for the $j$ th module representing clusters of couplings between muscles in space and time across trials respectively.

$$
\begin{gathered}
I_{\text {Space-Time }} \approx \\
\left(\begin{array}{ccc}
\left(m^{1} t^{1}\right) & \cdots & \left(m^{1} t^{L}\right) \\
\vdots & \ddots & \vdots \\
\left(m^{k} t^{1}\right) & \cdots & \left(m^{k} t^{L}\right)
\end{array}\right) \approx\left(\begin{array}{lll}
\boldsymbol{w}_{j}^{t^{1}} & \cdots & \boldsymbol{w}_{j}^{T^{L}}
\end{array}\right) \cdot\left(\begin{array}{c}
\boldsymbol{v}_{j}^{m^{1}} \\
\vdots \\
\boldsymbol{v}_{j}^{m^{k}}
\end{array}\right)+\text { residuals }
\end{gathered}
$$

Equation 3.3. 

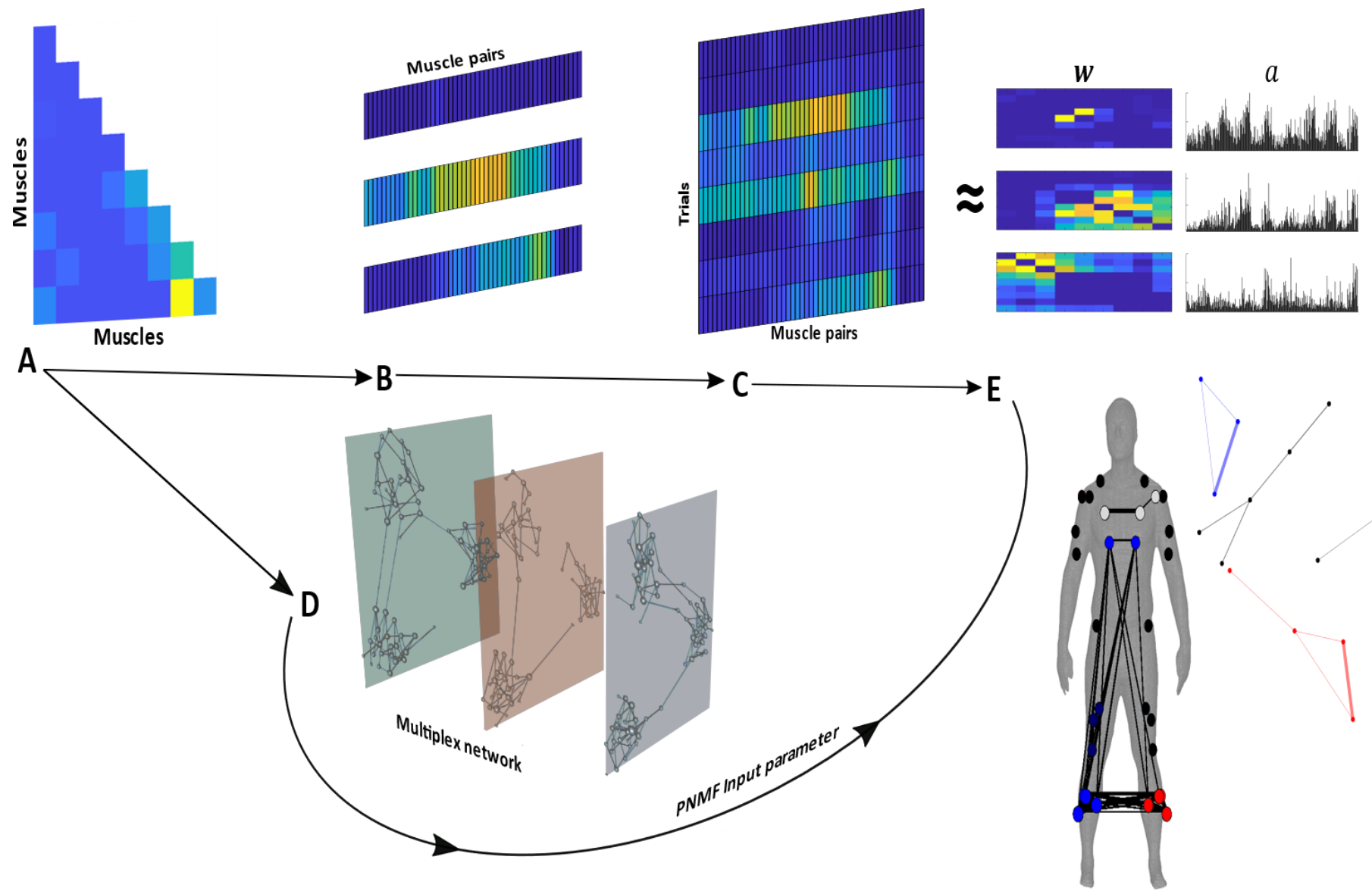

Fig. 2: (A) The GCMI is computed between each unique pair of muscles (spatial) or time-vectors (temporal) or trial-to-trial (space-time) vectors, creating a lower-triangular matrix. (B) The lower-triangular matrices are reshaped into single vectors and concatenated, creating the input matrix as shown in (C). (D) The matrices depicted in (A) are concatenated into a multiplex network and input into a generalised Louvain algorithm to identify the optimal community structure (See 'Model rank specification' section). (E) These input matrices shown in (C) are then factorized into lower-dimensional representations of $(D)$ here referred to as synergy weights $(\boldsymbol{w})$ and activation coefficients (a) using the optimal community structure as an input parameter in projective non-negative matrix factorisation (PNMF). The unique structure of this output for each of the synergy models is described in equations 3.1-3.3. A secondary community detection was then conducted to identify the submodular structure in the extracted synergies.

Spurious dependencies typically associated with noise may be found in the above computations and so it was necessary to apply a threshold to identify only the significant associations. A methodology derived from statistical physics, namely a modified percolation analysis [34], was chosen for this framework. This form of percolation analysis was developed to reconcile two opposing characteristics of biological networks, modularity (i.e. the extent of sparsity between groups of densely connected nodes) and 'small-world' properties (i.e. the integration of all nodes in a network towards the shortest average path length) $[34,35]$. The percolation analysis was shown to be effective in identifying functional modular structures while maintaining a sensitivity to the long-range connections that act as the bridges between modules which are often weaker in biological networks [36]. Moreover, this methodology is particularly suited to community detection procedures discussed in the 'Model-rank selection' section that follows [37]. By iteratively removing the weakest dependencies until the 'giant component' in the network starts to become effected, the network sparsification threshold can then be defined as this critical stopping point, providing a data-driven method to reduce the effect of noise that can be applied to specific networks.

\section{Model rank specification}

To represent muscle activations adequately, it is necessary to select an optimal number of components to extract. On the other hand, it is also necessary to maintain a model rank that produces physiologically and functionally meaningful synergies while also identifying a low-dimensional space, a challenging task within the literature [38]. To accommodate the unique characteristics of the GCMI matrices generated here, we developed a novel model rank selection criterion by defining a muscle synergy, a group of muscles acting cohesively as a functional unit, in networktheoretic terms. More specifically for the identification of these functional units in our data, we defined a synergy as a densely connected set of nodes otherwise known as a community [39]. Prior to dimensionality reduction using PNMF, the row for each trial of an input matrix was reverted back to an adjacency matrix and became a layer in a multiplex network with all other trials. In the case of space-time computations, the temporal pattern across each spatial vector 
bioRxiv preprint doi: https://doi.org/10.1101/2021.10.15.464450; this version posted October 20, 2021. The copyright holder for this preprint (which was not certified by peer review) is the author/funder, who has granted bioRxiv a license to display the preprint in perpetuity. It is made available under aCC-BY-NC 4.0 International license.

constituted the multiplex network. We implemented the percolation analysis described previously on each individual layer of the supra-adjacency matrix before the following computation.

Modularity in a monoplex network can be determined by the difference between the sum of all vertices that fall between all possible pairs of vertices in the network and that which would be expected by random chance [35]. This quantity is known as the Q-statistic which acts as an objective function to be optimised in community detection algorithms, ranging from 0-1 with 1 indicating maximal modularity $[35,40]$. The multiplex modularity, a generalisation of the Q-statistic to multi-layered networks, was implemented here using the generalized Louvain algorithm where the community structure of intra-layer edges between all nodes and all inter-layer edges between nodes representing the same muscle/timepoint could be considered [39,41]. The resolution parameter $\gamma$ was set to the default value of 1 for classical modularity [39]. The number of communities identified served as the input parameter for PNMF (Fig.2(E)). To verify the communities identified a priori were in fact extracted using PNMF, the maximum value in each row across synergy weight vectors $(\boldsymbol{w})$ served as an indicator for hard-cluster assignment [31]. By visually inspecting the cluster assignments produced by PNMF and those found through community detection, the output was found consistent (see Supplementary Fig. 1. for an example of this procedure).

\section{Trial-to-trial network configuration and task dependence of extracted muscle synergies}

Following the extraction of muscle synergies using the GCMI framework, we then investigated whether the identified sharing patterns also aligned with the other attributes of the synergy concept, i.e. reciprocal compensation/flexibility and task dependence [23]. As mentioned in the previous section, biological networks are generally characterised by two competing properties, fractal modularity and small-worldedness $[34,36]$. The scale invariance of network architectures allows for greater robustness to injury and adaptability while the efficient transfer of information is crucial for within- and across-network communication and cohesion $[42,43]$. It is therefore unsurprising to find modularity has been related to the motor learning process and small-worldedness to important integrative mechanisms such as working memory performance [44,45]. In the context of the synergies extracted here and their reorganisation, fluctuations in these network properties from trial-to-trial may provide interesting insight into the neural control of movement. This is supported by experimental findings where increased integration in the muscle space and the reorganisation of modular structure from trial-to-trial were observed with additional motor noise and adaptations to task constraints respectively $[46,47]$.

Thus, we sought to determine if the GCMI framework could capture the dynamic balance between modularity and small-worldedness from trial-to-trial in the extracted activation coefficients. We firstly determined the global efficiency (GE) and Q-statistic for modularity (Q) for individual layers of the thresholded multiplex network depicted in Fig.2(D). GE quantifies the small-worldedness of a network through the ratio of present to possible edges [48]. Q was determined for individual network layers using the conventional Louvain algorithm [40,48]. Analogous to previous work [49], this created two vectors in the spatial and temporal domains that were demeaned with respect to the task along with their corresponding activation coefficients. The correlations between single trial synergy activations and the network property measures defined above (GE, $\mathrm{Q}$ ) for a fixed task (noise correlations) were then computed using Pearson's correlation [49]. The strength and direction of these correlations therefore provide insight into the sensitivity of the extracted synergies to this network level trade-off. To further exemplify the fractal modularity of the synergies produced by this framework, a secondary community detection procedure was carried out on the synergy weights using the traditional Louvain algorithm $[40,48]$. The cluster assignment of these submodules were then represented on a human body model by the colour of nodes on the network [50].

To determine if the information encoded in the extracted muscle synergies can discriminate between the different experimental conditions/tasks, we computed the GCMI between individual spatial or temporal activation coefficient vectors and the reaching task variable. To assess whether the task-discriminative information presented in each component was significant, activation coefficients were also randomly shuffled and the GCMI with respect to the task was calculated. This procedure was repeated 100 times and the $95^{\text {th }}$ percentile of these collective values acted as an empirical threshold for significance. The specific task attributes for each dataset analysed in this study are detailed in the 'Experimental design and setup' section.

It is crucial to the utility of this framework that the muscle synergies extracted are consistent across a set of participants who have conducted the same motor tasks. The same extraction procedures were therefore conducted 
across the remaining participants in each dataset and the following analysis was conducted to determine the structural similarity. Muscle synergies were first visually inspected and compared across participants and those with a similar functional interpretation were paired together. The muscle synergies in each of the pairs were vectorized and the Pearson's correlation was found for each pairing. This produced a set of coefficients that were transformed into Fisher's Z values, the average and standard deviation of which was taken and reverted to correlation coefficients. This value served as a global index of similarity across participants. To extract the average muscle synergy from each model, the synergies across participants in each dataset were vectorized and the mean network was then found. To eliminate individual differences from the average synergies, the percolation analysis sparsification procedure described above was conducted.

\section{Experimental design and data collection}

\section{Dataset 1}

A dataset of EMG activity recordings was generated across seven healthy, right-handed adults who provided informed consent to participate in the experiment that conformed to the declaration of Helsinki (approved by the local ethical committee ASL-3 ("Azienda Sanitaria Locale," local health unit), Genoa). Participants were instructed to perform center-out (forward, denoted by $f w d$ ) and out-center (backward, denoted by $b w d$ ) one-shot point-to-point movements between a central location (P0) and 4 peripheral locations (P1-P2-P3-P4) evenly spaced along a circle of radius $40 \mathrm{~cm}$ at a fast pace $(370-560 \mathrm{~m} / \mathrm{s}$ duration on average across participants). 40 repetitions per target/speed were captured per participant. Movement onset and offset was defined as points in which the velocity profile (captured via the kinematic data of a passive marker (Vicon, (Oxford, UK)) placed on the finger-tip that was numerically differentiated) superseded $5 \%$ of its maximum. The targets to which the participants reached were circles of radius 0.2 $\mathrm{mm}$ which they had to touch. No restraints or supports were provided while the order of the tasks were randomized.

A

A

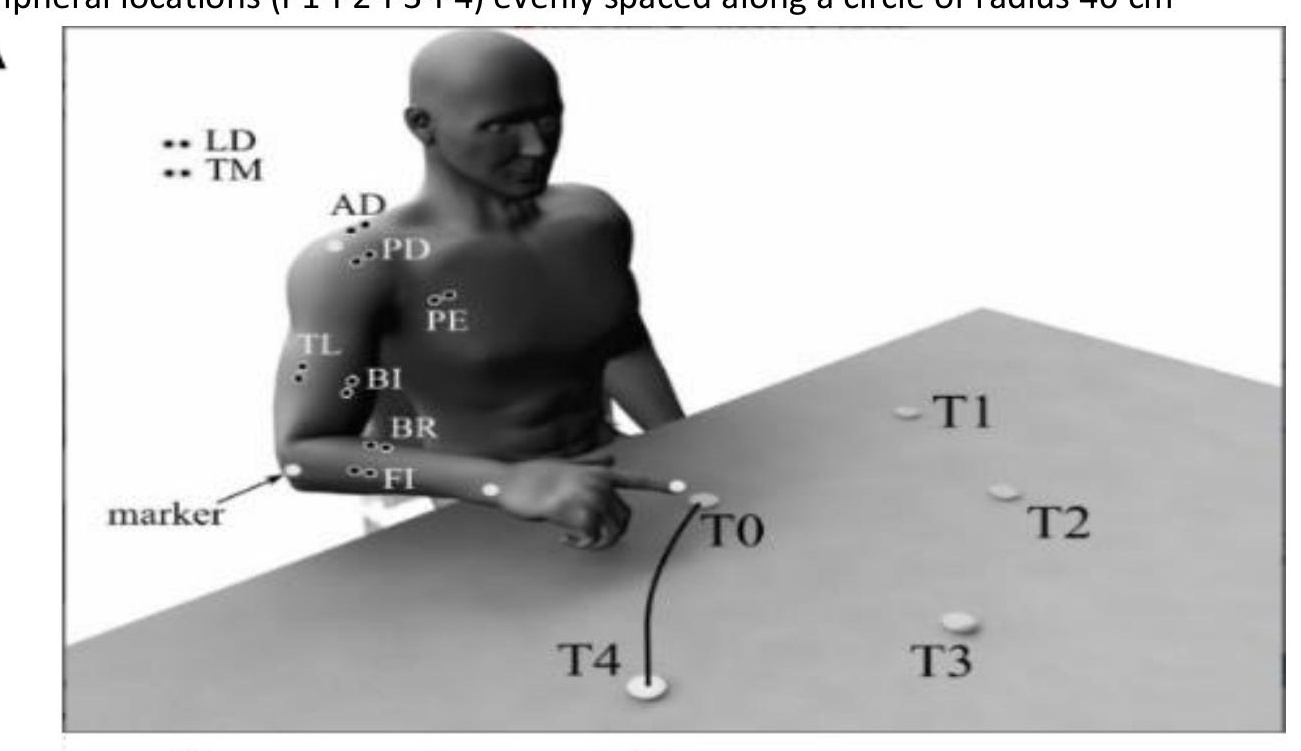

B
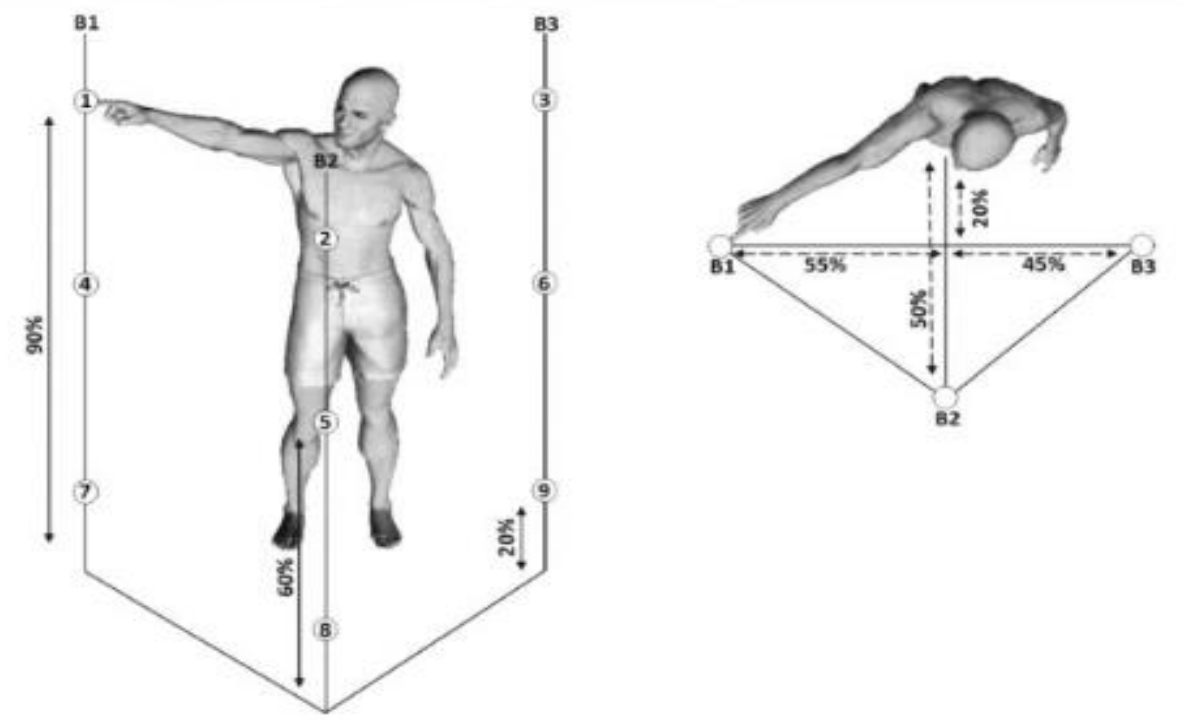

Fig.3 (A): An illustration of the experimental setup for Dataset 1.40 repetitions for each target were conducted while the EMG activity from the following muscles was recorded: LD: Latissimus dorsi; AD: Anterior deltoid; PD: Posterior deltoid; PE: Pectoralis; TL: Triceps lateral; BI: Biceps brachii; TM: Triceps medial; BR: Brachioradialis; FE: Finger extensor. (B): An illustration of the experimental setup for Dataset 2. Participants were asked to perform wholebody pointing movements between pairs of targets. Included were 9 different targets at 3 different heights, creating 72 possible movement tasks for analysis. 
bioRxiv preprint doi: https://doi.org/10.1101/2021.10.15.464450; this version posted October 20, 2021. The copyright holder for this preprint (which was not certified by peer review) is the author/funder, who has granted bioRxiv a license to display the preprint in perpetuity. It is made available under aCC-BY-NC 4.0 International license.

Simultaneously, the activity of nine upper-body and arm muscles was recorded as presented in Fig.3(A) (Aurion (Milan, Italy)). The EMG setup was implemented in line with SENAIM guidelines. EMG signals were digitized, amplified (20- $\mathrm{Hz}$ high-pass and $450-\mathrm{Hz}$ low-pass filters), and sampled at $1,000 \mathrm{~Hz}$ (synchronized with kinematic sampling). Subsequently, to extract the signal envelopes, the EMG signals were processed offline using a standard approach [13]: the EMGs for each sample were digitally full-wave rectified, low-pass filtered (Butterworth filter; 20-Hz cut-off; zerophase distortion), normalized to 1,000 time points and then the signals were finally integrated over 20 time-step intervals yielding a final waveform of 50 time-steps. For dataset 1 participants, the task dependence of individual spatial and temporal $\boldsymbol{a}$ was determined against discrete variables representing all point-to-point movements (P1-P8), all center-out (forward) movements (P1-P4), all out-center (backward) movements (P5-P8) and the movement direction ( $f w d$ vs. bwd).

\section{Dataset 2}

The EMG activity of 30 muscles from four healthy, right-handed adults (Age $=25 \pm 3$ years, height $=1.72 \pm 0.08$ meters, weight $=70 \pm 7 \mathrm{~kg}$ ) was recorded (Aurion system, (Milan, Italy), SENAIM guidelines) while participants performed whole-body point-to-point movements in various directions and at differing heights. The muscles recorded included: tibialis anterior, soleus, peroneus, gastrocnemius, vastus lateralis, rectus femoris, biceps femoris, gluteus maximus, erector spinae, pectoralis superior, trapezius, anterior deltoid, posterior deltoid, biceps and triceps brachii on both sides. The experimental setup (Fig.3(B)) was approved by the Dijon Regional Ethics Committee and conformed to the Declaration of Helsinki. Written informed consent was obtained by the participants following guidelines set by the Universite de Bourgogne. This setup is described in detail elsewhere and will therefore be outlined briefly here [51]. Participants were asked to perform whole-body pointing movements between pairs of targets ( 9 targets at three different heights $=72$ different movement tasks). Over the course of two separate sessions, participants performed 15 repetitions of each movement ( 30 repetitions in total), resulting in $72 \times 30=2160$ trials per participant. The processing of EMG recordings followed the same standardized protocol conducted on Dataset 1 while onset and end times for individual trials were determined in a similar manner using the kinematics of the pointing index finger at a frequency of $100 \mathrm{~Hz}[13,52]$. The left arm of participants was unconstrained and at rest throughout each trial while the order of movements was randomised. The resulting data was pooled, creating (50 time-point $\times 2160$ trials) $\times 30$ muscle matrices. For dataset 2 participants, the task dependence of individual spatial $\boldsymbol{a}$ was determined against discrete variables representing the spatial movement features, i.e. start- and end-point bar and height (see fig.3(B)), up-down and left-right reaching directions. For individual temporal $\boldsymbol{a}$, the task dependence was determined with respect to temporal movement features representing the evolution of movement in time, i.e. start-point, reaching direction and end-point target.

\section{Results}

Muscle synergies in point-to-point reaching movements

\section{Spatial synergy model}

To identify spatial synergies, i.e. muscle couplings across time within each trial (see Fig. 1A), we applied the proposed framework to Dataset 1 consisting of arm-reaching movements in 8 different directions. In brief, we first computed GCMI between all pairs of muscles for each trial. We then input the single-trial GCMI values of all muscle pairs to the PNMF algorithm to identify couplings between muscles, i.e. muscle synergies, which are consistent across trials (see Fig. 2 for an illustration of the full methodology).

Taking a representative, example participant, three distinct spatial synergies were found using the community detection procedure (see Materials and Methods for details) with a Q-statistic value of 0.998 (Fig.4(A)). A high coactivation between the medial and lateral triceps was found in S1 along with a lower co-activation of the anterior deltoid. S2 represented a more global activation across the upper-arm and shoulder muscles while the finger extensor and brachioradialis remained inactive. S3 contained couplings between the finger extensor, brachioradialis and biceps brachii. Taking these together, one can interpret their functional grouping as the following: S1 represents elbow extension, S2 proximal stabilisation/shoulder flexion, and S3 is forearm flexion/finger control.

To assess the task-relevance and organisation of the identified synergies, we computed the task information conveyed by the synergy recruitment on each trial (Fig.4(B)) and the noise correlation between synergy recruitment and essential network properties derived prior to dimensionality reduction (Fig.4(C)). Forward vs. backward movements were mainly discriminated by S2 activations ( 0.26 bits) which implement shoulder flexion. S1 (elbow 
extension) contained the most information for movement direction across all movements, i.e. P1-P8 (0.51 bits), but also when considering only forward (P1-P4, 0.45 bits) or only backward (P5-P8, 0.52 bits) movements. S3 activations (predominantly of forearm muscles) did not contribute significantly to discrimination of forward movements (P1-P4, 0.16 bits) or forward vs backward direction ( 0.033 bits) but carried significant information for backward movements (P5-P8, 0.34 bits) which contributed to the overall discriminatory information (P1-P8, 0.29 bits). We also found significant correlations between all three synergy activation coefficients and network-theoretic measures $(p<0.05)$ but in opposing directions with positive associations for integration (GE) and negative associations for segregation (Q). Both S2 and S3 shared the highest association with $G E(R=0.72)$, an interesting finding considering these synergies represent the distinct functional grouping of upper- and lower-arm muscles respectively. The activation in S1 reduced the least with positive changes in modularity $(R=-0.11)$, likely due to the specificity of this synergy to the medial- and lateral-triceps.

A secondary community structure analysis of the extracted synergies revealed two submodules in S1 and S2 and just one submodule consisting of the lower-arm musculature in S3. In S1, these submodules consisted of the medial- and lateral triceps (green) and anterior deltoid and latissimus dorsi (pink). The medial-triceps and posterior deltoid (green) and lateral-triceps, pectoralis major and anterior deltoid (blue) comprised the S2 submodules.

A)

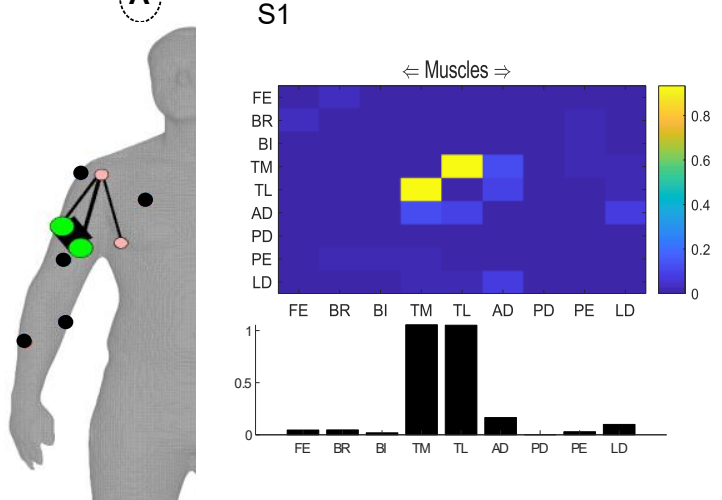

S2
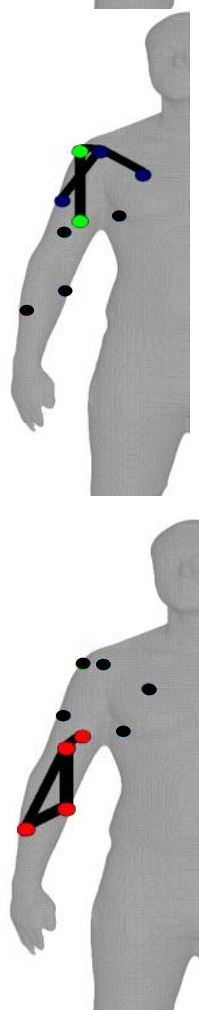

B
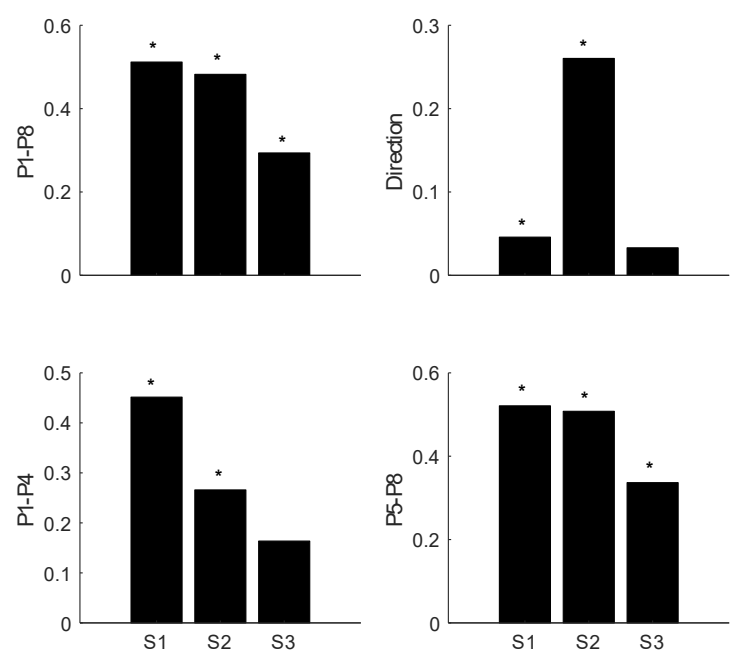

(c)
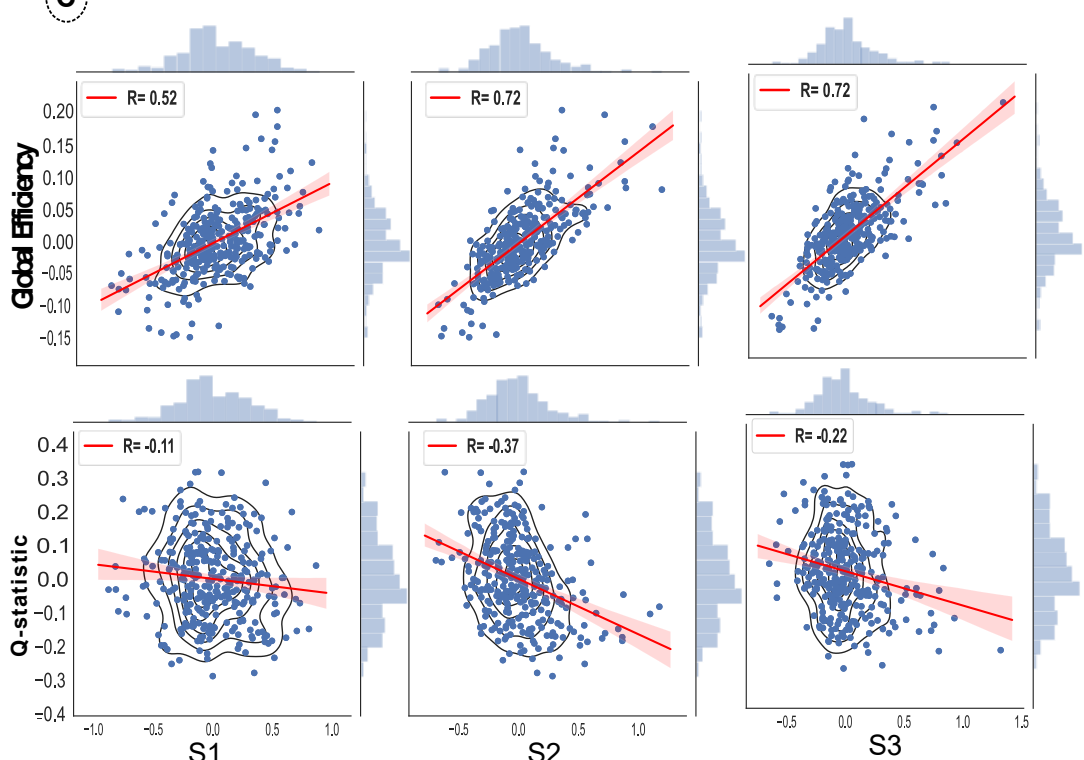

Fig 4: (A) The spatial synergies extracted from an example participant in Dataset 1. The community detection criteria found three distinct communities that were extracted using PNMF. The bar graph below represents the average values in each column of the adjacency matrix. The human body model illustrates the values in the adjacency matrix with the width of the edges and size and colour of the nodes providing insight into connection strengths, submodular structure and involvement respectively [50]. Submodular structure was identified using the conventional Louvain algorithm on the synergy matrices [40]. Unconnected nodes are in black. (B) The encoded information of the three spatial synergies identified in the example participant in dataset 1 for four task attributes: P1-P8, P1-P4, P5-P8 and Direction. Significant information (p<0.05) is indicated with *.(C) The correlation between trial-to-trial fluctuations in Global Efficiency/Q-statistic for modularity and noise in the spatial activation coefficients. 
bioRxiv preprint doi: https://doi.org/10.1101/2021.10.15.464450; this version posted October 20, 2021. The copyright holder for this preprint (which was not certified by peer review) is the author/funder, who has granted bioRxiv a license to display the preprint in perpetuity. It is made available under aCC-BY-NC 4.0 International license.

\section{Temporal synergy model}

To characterise the temporal structure of muscle co-activations, we also applied the GCMI framework in the temporal domain, returning three temporal synergies (Fig.5(A)) for the same example participant that captured temporal associations across muscles. This was produced by computing GCMI between pairs of time-vectors (vectors of activations across all muscles at different timepoints, see Fig.1B) within each trial, modelling the output as a multiplex network to identify the optimal community structure and finally extracting these communities using PNMF.

T1 presented dependencies between timepoints for the initial phase of the reaching movement. These dependencies are accompanied by a high dependency between the final $\left(49^{\text {th }}-50^{\text {th }}\right)$ timepoints that is connected to this initial burst through moderate dependencies among adjacent timepoints along the diagonal. T2 illustrates a prominent clustering of dependency from timepoints 15-25 and continuing with strong connection strengths within this timepoint range for the remainder of the movement. T3 presented high dependencies with all timepoints from approximately half-way into the movement until the endpoint.

(A)

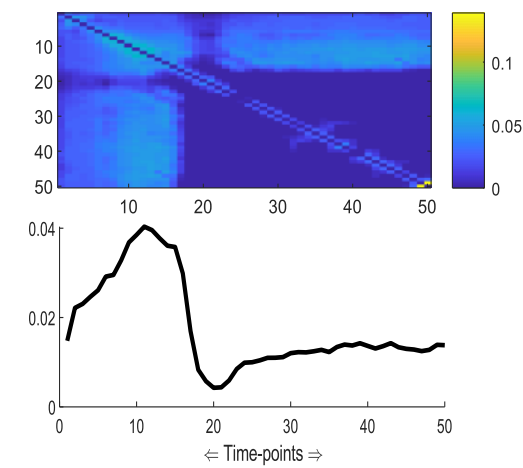

$\mathrm{T} 2$
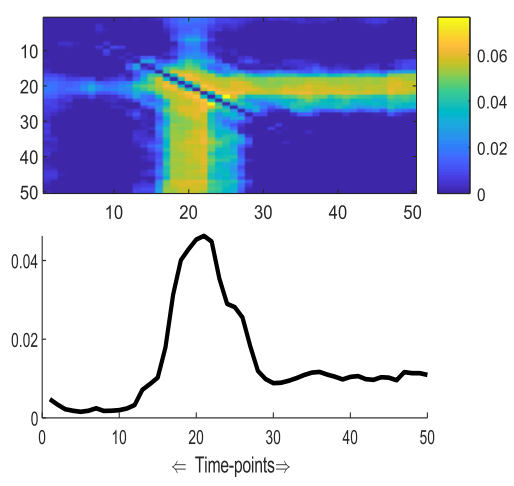

T3

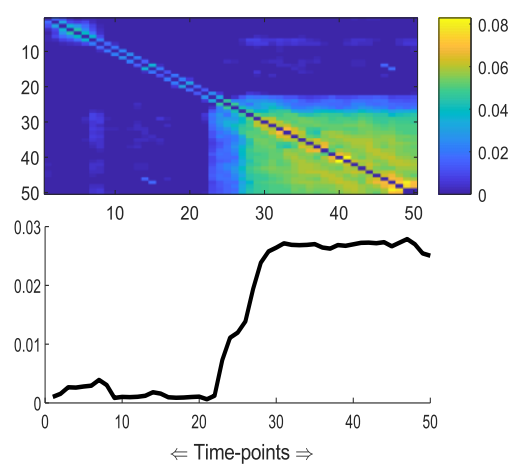

(B)
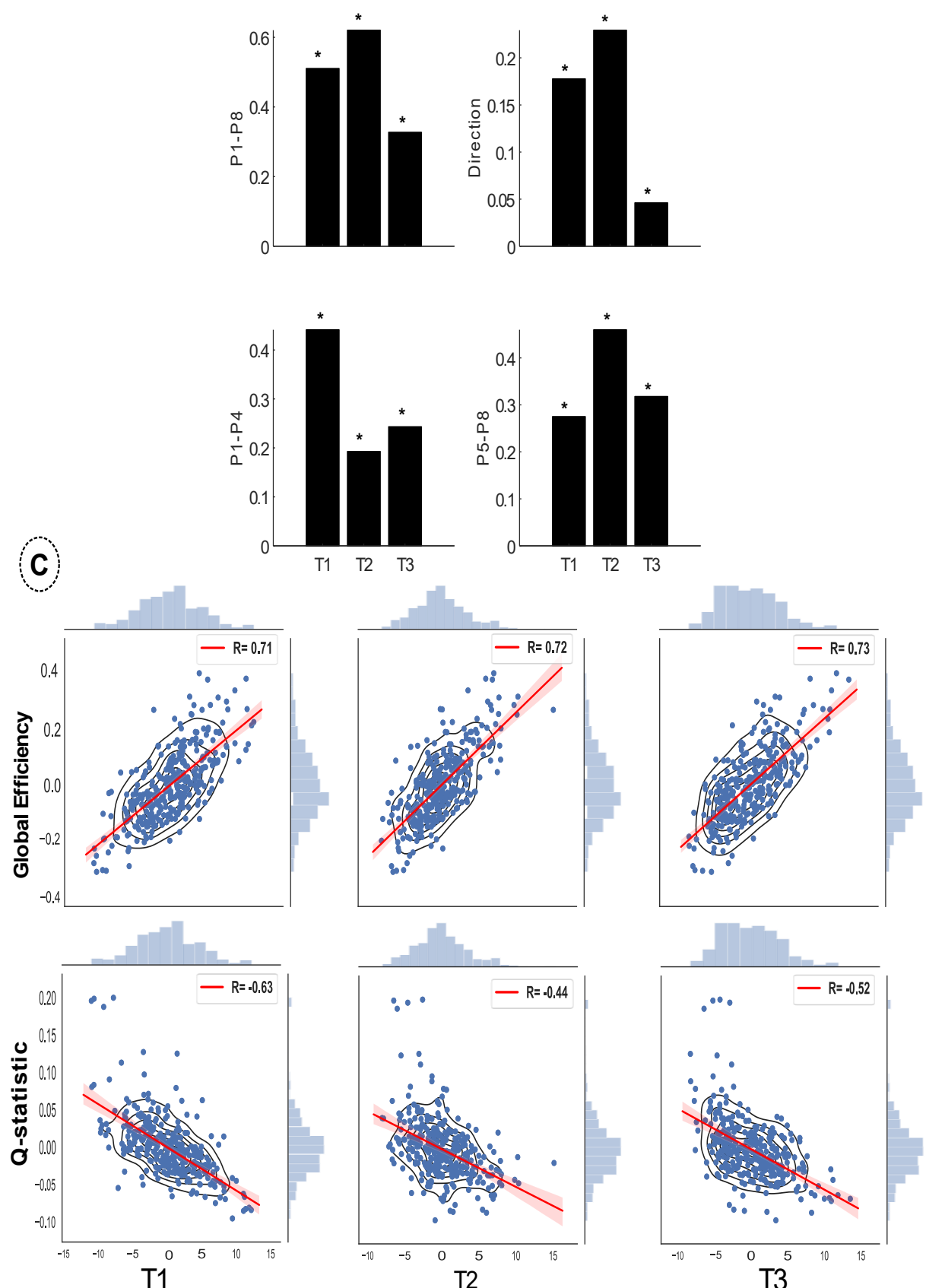

T3

Fig.5:(A) The temporal synergies from the example participant in dataset 1 . The community detection criteria found three distinct communities that were extracted using PNMF. The line plot below represents the average values within each column of the adjacency matrix. (B) The encoded information of the three temporal synergies identified in the example participant in dataset 1 for four task attributes: P1-P8, P1-P4, P5-P8 and Direction. Significant information $(p<0.05)$ is indicated with *. (C) The correlation between trial-to-trial fluctuations in Global Efficiency/Q-statistic for modularity and noise in the temporal activation coefficients. 
bioRxiv preprint doi: https://doi.org/10.1101/2021.10.15.464450; this version posted October 20, 2021. The copyright holder for this preprint (which was not certified by peer review) is the author/funder, who has granted bioRxiv a license to display the preprint in perpetuity. It is made available under aCC-BY-NC 4.0 International license.

Taken together, a single tonic synergy (T1) was accompanied by a bi-phasic pattern of activation (T2 (acceleration) and T3 (deceleration)) that explained point-to-point reaching movement in this example participant. This observation is supported by an analysis we conducted on the task-dependency of the underlying activation coefficients (Fig.5(B)). Consistent with the differing functional roles of these temporal synergies, differences between forward movements (P1-P4 differing in the respective end-points) were predominantly captured by T1 (peaking at the final time points) whereas differences in backward movements (P5-P8 differing in the respective starting points) were predominantly captured by T2 (activating earlier in the movement) which contained the most information overall (P1P8, 0.62 bits and direction, 0.23 bits). The noise correlations between all synergy activations and both GE and $Q$ were significant $(p<0.05)$ and in opposing directions, with all synergy activations positively related to GE and negatively associated with $\mathrm{Q}(\mathrm{Fig} .5(\mathrm{C}))$. The tonic synergy (T1) was most sensitive to changes in modularity from trial-to-trial $(\mathrm{R}=$ $-0.63)$, while $T 3$ was highest in its association with $G E(R=0.73)$. The sensitivity to integration in the latter case is likely reflective of the differing muscular involvements required at each reaching end-point position.

\section{Space-Time synergy model}

As an extension of the spatial and temporal synergy models, we concurrently extracted these distinct modules in a unifying space-time model, capturing distinct couplings in trial-to-trial dependencies that are consistent across spatial and temporal components. To do this, we first computed GCMI across all muscle pairs and time points and then decomposed the identified dependencies into components with distinct spatial and temporal signatures. For the same example participant in dataset 1 , we determined an optimal model-rank of three using the community detection procedure with a Q-statistic of 0.97 . Fig.6 below illustrates this output with the spatial and temporal synergies corresponding on a 1:1 basis across each row. In other words, the space-time model identified three synergies and characterised their spatial and temporal structure across all trials. The spatial synergies in ST2 and ST3 are relatively robust with those derived from the spatial synergy model previously (Fig.4), demonstrating the consistent coupling of the medial and lateral triceps and within the upper- and lower-arm musculature respectively. Their corresponding temporal synergies represent muscle co-activation throughout the movement and an early-and-late phasic activation that is dynamically involved in the flexion/extension of reaching movements respectively. ST1 presents a strong dependency between the anterior deltoid and latissimus dorsi that is active at the beginning and end of the movement and relatively quiet in the mid-range. This synergy is likely reflective of the alternate roles the anterior deltoid and latissimus dorsi carry out in stabilising the shoulder joint during forward and backwards reaching movements. A secondary community detection procedure revealed two submodules in each of the spatial synergies that are represented by the colours of individual nodes in the human body model networks. For ST1, the submodules included the anterior deltoid, latissimus dorsi and both the medial- and lateral triceps (cyan) and the finger extensor and brachioradialis of the lower-arm (red). In ST2, the medial- and lateral triceps (cyan) and a long range connection between the brachioradialis and posterior deltoid (blue). Finally, ST3 consisted of the medial-triceps and posterior deltoid (blue), and lateral-triceps and anterior deltoid (green). 
bioRxiv preprint doi: https://doi.org/10.1101/2021.10.15.464450; this version posted October 20, 2021. The copyright holder for this preprint (which was not certified by peer review) is the author/funder, who has granted bioRxiv a license to display the preprint in perpetuity. It is made available under aCC-BY-NC 4.0 International license.

\section{ST1}
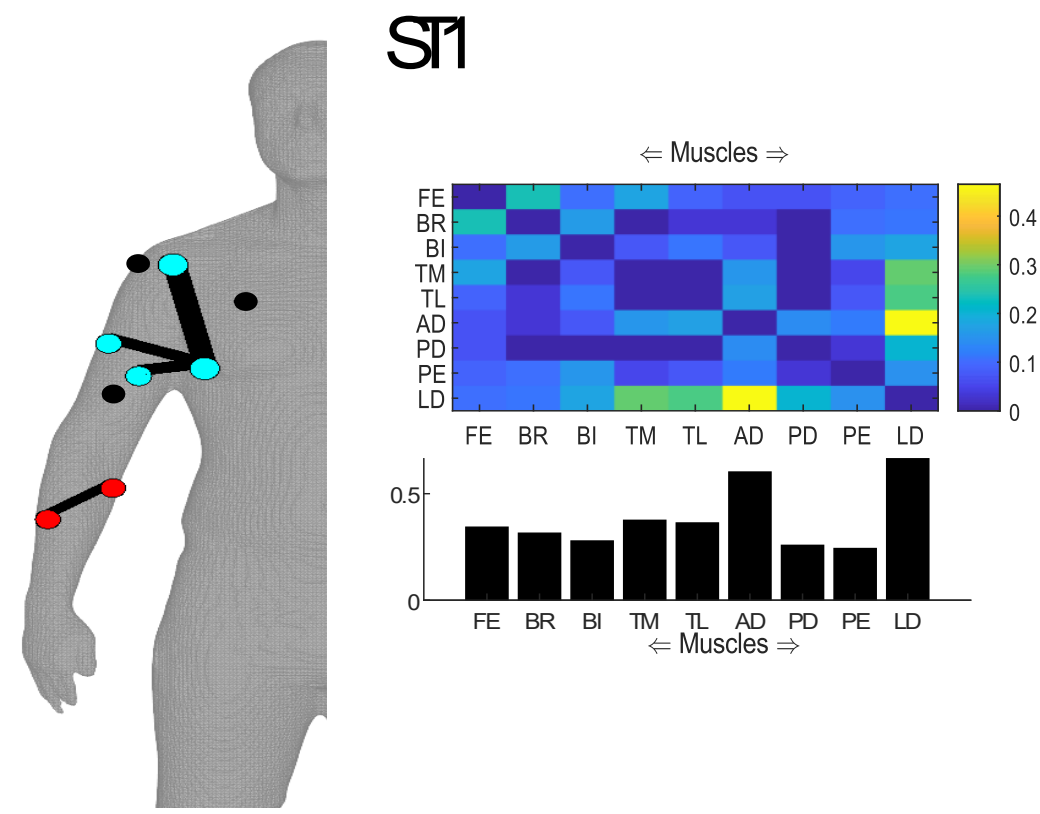

$\begin{array}{llllllllll} & \text { FE } & B R & B I & T M & T L & A D & P D & P E & L D\end{array}$

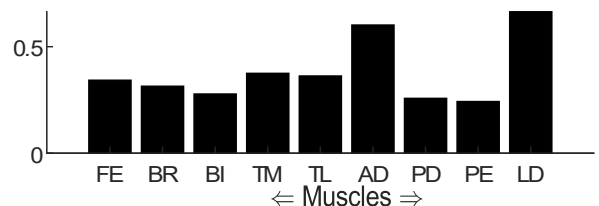

SR
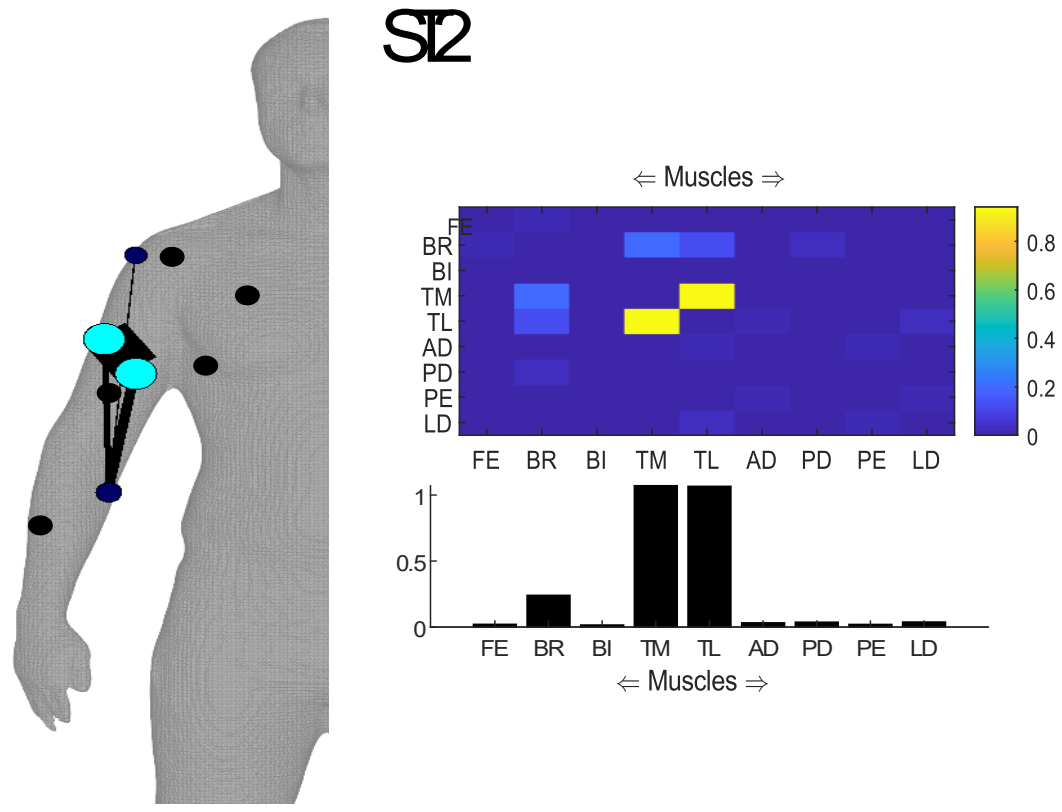

SB
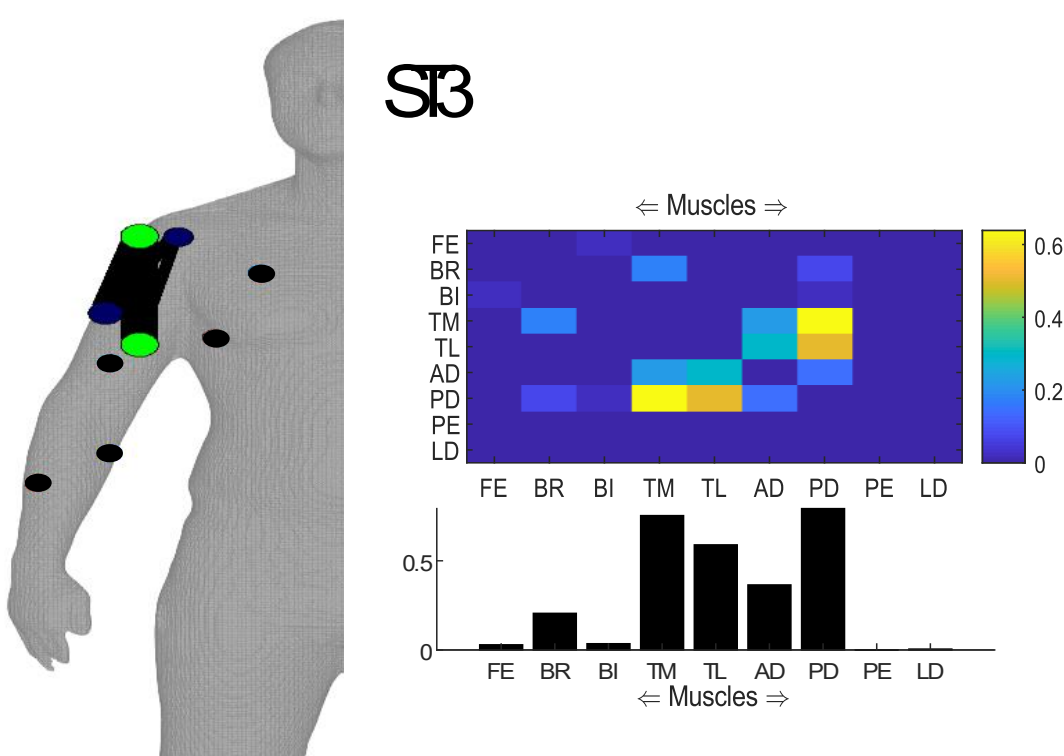
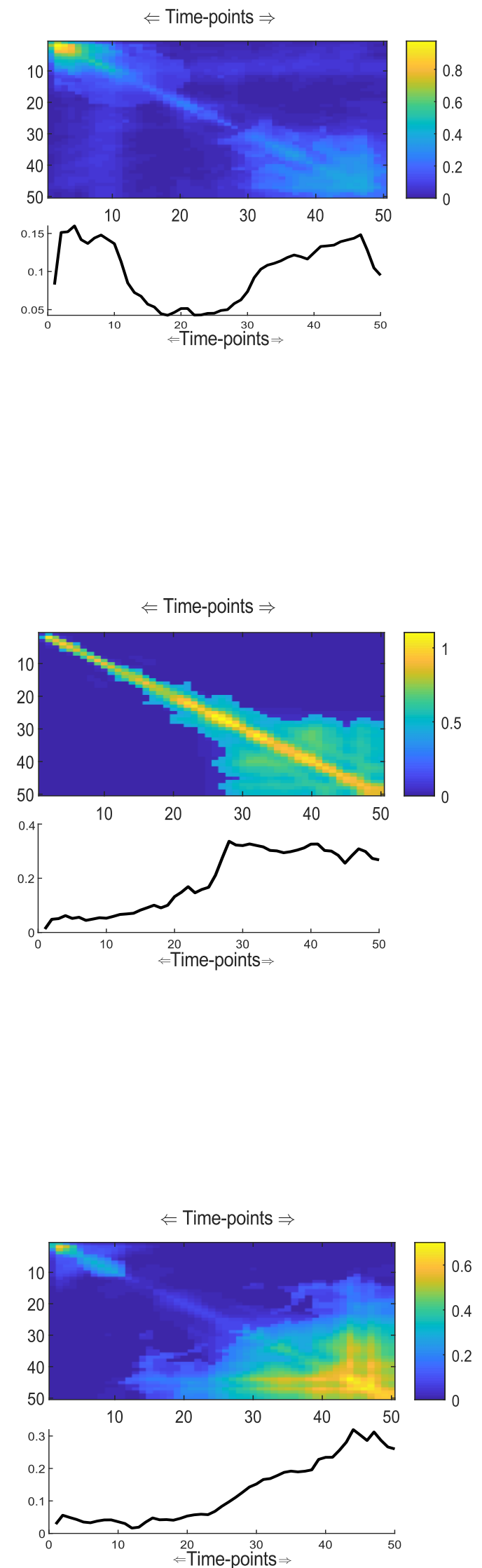

Fig.6: The Space-Time synergies extracted from the example participant in dataset 1. Three communities were identified a priori and then extracted using PNMF. Spatial and temporal synergies correspond on a 1:1 basis here as presented across rows. The bar/ line plots below represents the average values within each column of the corresponding adjacency matrix. The connection strengths, submodular structure and involvement of nodes are indicated by the human body model via the edge widths, node colour and size respectively [50]. Submodular structure was identified using the conventional Louvain algorithm on the synergy matrices [40]. Unconnected nodes are in black. 
bioRxiv preprint doi: https://doi.org/10.1101/2021.10.15.464450; this version posted October 20, 2021. The copyright holder for this preprint (which was not certified by peer review) is the author/funder, who has granted bioRxiv a license to display the preprint in perpetuity. It is made available under aCC-BY-NC 4.0 International license.

\section{Consistency across participants (Dataset 1)}

We then sought to determine the similarity of the spatial, temporal and space-time synergies extracted using the GCMI framework across participants. This was conducted by computing a similarity index between pairs of functionally similar synergy weights derived at a representative model rank for the dataset and the average \pm standard deviation was found (see Materials and Methods section). Within Dataset 1, we identified 2.4 spatial communities on average (range $=2-3$ ) with a mean Q-statistic of 0.997 and threshold value of 0.13 bits (Supp. Fig.2). A satisfactory level of consistency was found $(R=0.78 \pm 0.37)$. S1 here represents elbow extension primarily with some moderate dependencies across the other muscles. S2 involves a more global activation but particularly between the medial and lateral triceps and between the anterior deltoid and pectoralis. S1 was typically higher in its task information across participants for direction ( 0.11 bits) and P5-P8 (0.23 bits) while S2 was prominent for P1-P8 (0.33 bits) and P1-P4 (0.24 bits). The contrasting relationship between the synergy activations and GE and $Q$ was replicated across participants with $\mathrm{S} 1$ presenting the strongest noise correlation for both network properties.

We identified an average of 3.4 communities (range $=3-4$ ) with an accompanying maximal modularity of 0.93 and threshold value of 0.46 bits on average in the temporal domain across participants in dataset 1 . The similarity index revealed a high level of correlation $(R=0.83 \pm 0.45)$ although with significant variability. Supplementary materials Fig.3(A) illustrates the three representative synergies taken from this procedure along with their mean task-encoded information. T1 presented a low-to-moderate level burst of dependency at the initial phase of movement, but a high dependency in the last two timepoint pairings. T2 had a more idiosyncratic pattern of dependencies across the movement with several bursts along the diagonal. T3 demonstrated a step in activation from the $30^{\text {th }}$ timepoint approximately with dependencies shared across timepoints for the remainder of the movement. In terms of task dependence, T1 was predominant for P1-P8 ( 0.31 bits) and P1-P4 ( 0.22 bits) and also highest for P5-P8 (0.23 bits). T2 contained the highest average task information for forward vs. backward directions ( 0.11 bits). T1 demonstrated the strongest correlation for both $G E(R=0.86)$ and $Q(R=-0.57)$ while T3 was weakest in its noise correlation with $Q(R=-$ $0.16)$.

For the space-time model, we determined that a model rank of three was representative of dataset 1 participants with a correlation of $0.69 \pm 0.33$ found (Supp. Fig.4). The Q-statistic was consistently high across participants, ranging from $0.89-0.99$ while the mean threshold value was 0.048 bits. All temporal synergies presented a high dependency between adjacent timepoints and along the diagonal, indicating that co-activations during reaching movements in various directions and points were most consistent across participants when considering trial-to-trial dependencies. The spatial synergies were consistent with those reported already here with ST2 capturing the characteristic dependency between the medial and lateral triceps and the ST3 spatial synergy the dynamic involvement of the upper-arm musculature during reaching. ST1 involved a combination of the anterior deltoid and pectoralis or latissimus dorsi.

\section{A generalisation to whole-body point-to-point reaching movements.}

We then sought to generalise the results presented above to a more complex dataset consisting of EMG activity from 30 muscles during whole-body point-to-point reaching movements at various heights and in various directions ( 82 distinct movements in total, each repeated 30 times) among 5 participants. This new high-dimensional dataset serves to demonstrate the applicability of the proposed framework to characterise the structure of large-scale EMG recordings during 3-dimensional unconstrained movements.

Spatial synergy model

Taking an example participant from dataset 2, we identified three spatial synergies with a maximal modularity of 0.994 (Fig. 7). S1 and S2 appear to represent the postural stabilisation activity related to normal stance and during point-topoint reaching respectively. This is indicated in S1 by the greater dependencies found in the lower-limbs and in the lower-limbs and left upper-body in the S2. S3 then completes this picture of whole-body reaching with significant dependencies clustered in the right upper-body. The dependency of these synergies across a number of task attributes including end-point bar and height, start-point bar and height along with the Up-down and left-right directions are also illustrated (Fig.7(B)), and support these functional interpretations. S1 (activating mainly the lower body) carried significant information about the horizontal dimension of movement (start and endpoint bar and left-right displacements) suggesting that its functional role was to drive body rotations. S2 (activating the left upper-body together with parts of the lower body) contained a higher level of task information, mostly for the vertical movement dimension (end-point height [0.242 bits] and the Up-down direction [0.208 bits]) suggesting that its functional role was to support vertical body displacements. Although task information was relatively high for S3 (right arm raising), 
just one task attribute was found to be significant (end-point height, 0.53 bits), suggesting that this synergy was relevant but highly variable in the information it contained. This could potentially be attributed to the highly variable demands required during upwards vs. downwards reaching movements for example where passive mechanics can be exploited in the latter but not the former. All synergy activations were significant in their trial-to-trial correlations with integrative and segregative network properties $(p<0.05)$, and presented contrasting directions in their association, exemplifying the trade-off between these properties. $\mathrm{S} 2$ demonstrated the greatest sensitivity to changes in $\mathrm{GE}(\mathrm{R}=$ 0.62 )and $Q(R=-0.41)$ in its underlying activations. Coinciding with the highly variable task information, $S 3$ presented the least sensitivity to changes in $G E(R=0.29)$ and $Q(R=-0.07)$.

The submodular structure of these synergies, represented on the human body model by the colour of the nodes shows four submodules for S1 (both erector spinae and the right tibialis musculature (blue), right femoral musculature (navy), left tibialis musculature (red) and both pectoralis major and the left anterior deltoid (white)) three submodules for S2 (left arm musculature and right tibialis anterior (pink), right arm and left femoral musculature (grey) and right femoral musculature (white)) and two submodules for S3 (upper-body musculature (white) and three right femoral muscles (pink)).

(A)

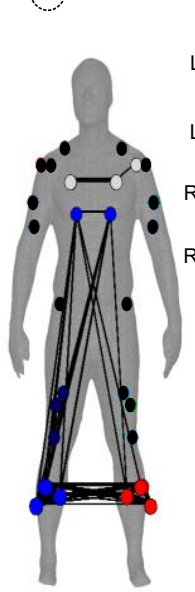

S1

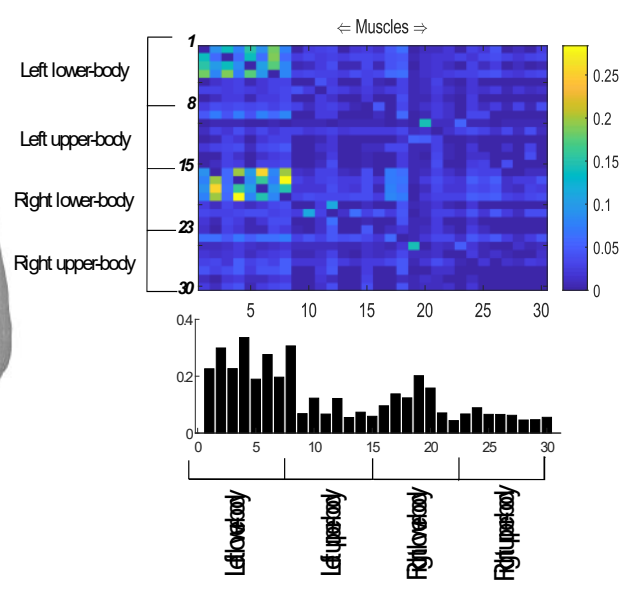

S2
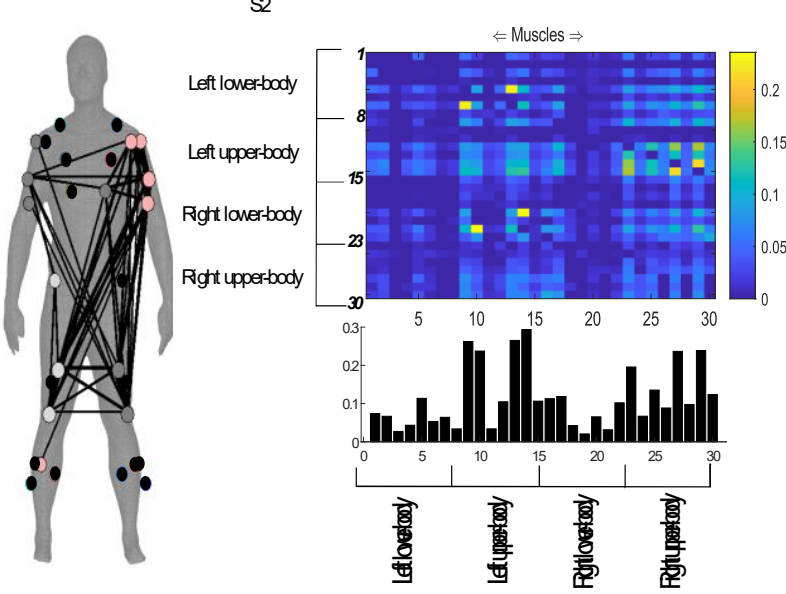

S3
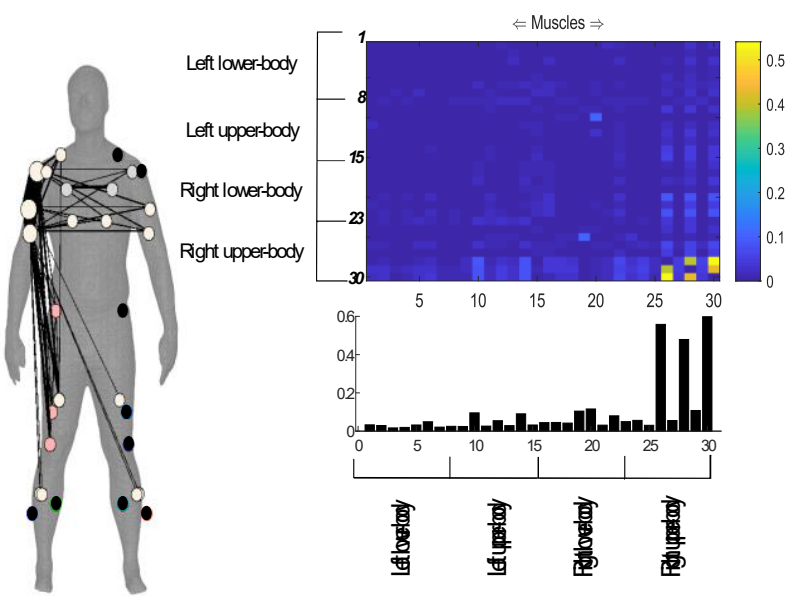

(B)
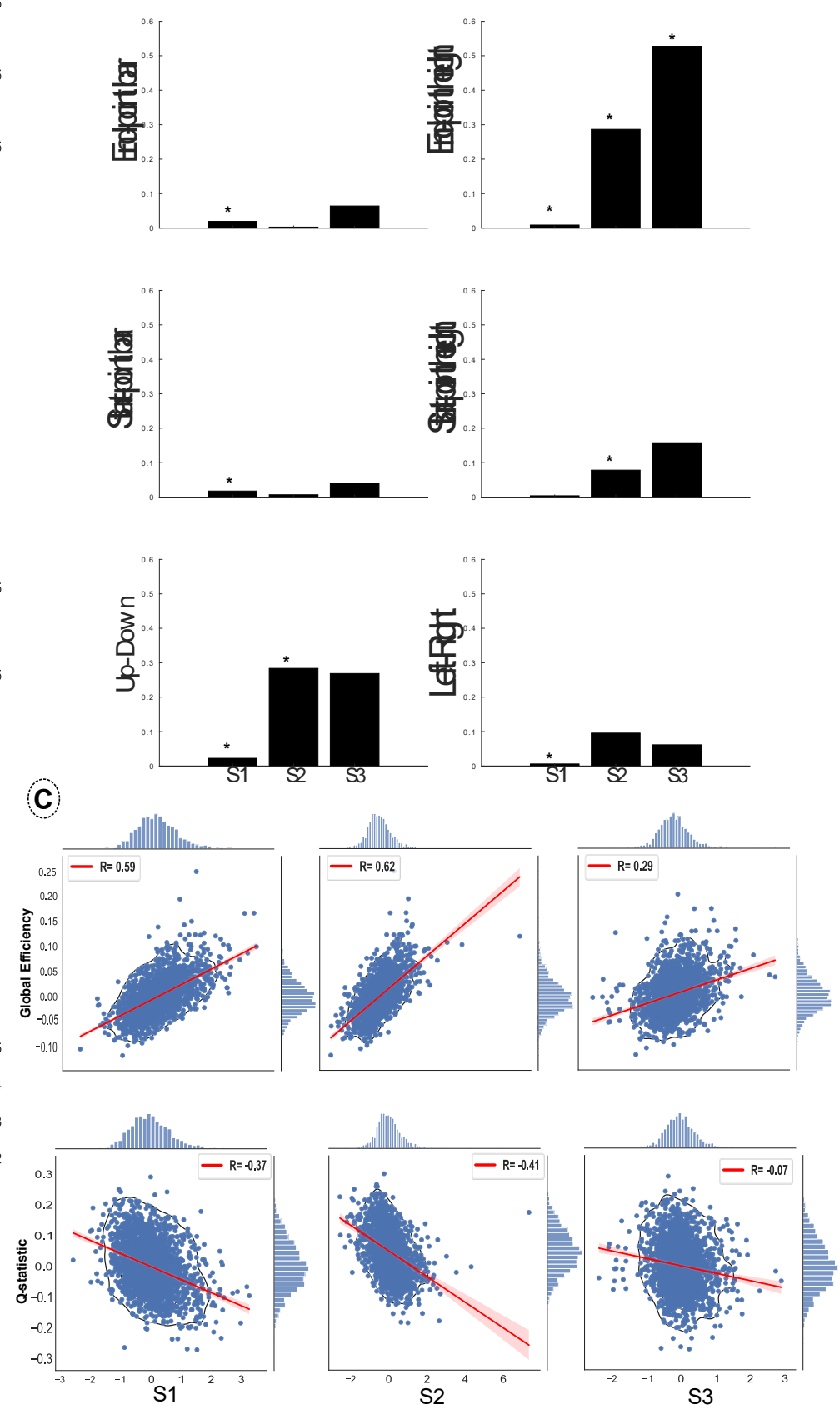

Fig.7: (A) The spatial synergies extracted from the example participant in dataset 2. The adjacency matrices are organised so that rows 1-8: left 
lower-limb, rows 9-15: left upper-body, rows 16-23: right lower-limbs and rows 24-30: right upper-body. The width of the edges on the human body model and the size and colour of the nodes indicate the connection strength, node involvement and submodular structure respectively [50]. Submodular structure was identified using the conventional Louvain algorithm on the synergy matrices [40]. Unconnected nodes are in black. (B) The information encoded for six task attributes is presented. ${ }^{*}$ indicates significance at $p<0.05$. (C) The noise correlation between spatial synergy activations and trial-to-trial network properties Global Efficiency/Q-statistic for modularity.

\section{Temporal synergy model}

To test how consistent the temporal synergies are when considering more complex motor tasks, we applied the temporal synergy model to the example participant in dataset 2, revealing three temporal synergies (Fig. 8(A)). T1 here contained moderate proximal dependencies along the diagonal and a sharp step to a new activation level near movement termination. The initial phasic activation of 72 here is more drawn out along the diagonal and less dependent on timepoints later in the movement. This is likely due to the different heights and directions in point-topoint reaching introduced in this experimental setup, requiring more variable onset-offset timing of phasic muscle activations. T3 consists of a late burst from timepoint 30-40 approximately. We found that all of these temporal synergies were significant for the three task attributes analysed here including start- and end-point and direction (Fig.8(B)). T3 contained the most information regarding the direction of reaching ( 0.13 bits). All 3 synergies contained similar amount of information about end-point position $(0.11,0.1$ and 0.11 bits respectively) while T2 was predominant for start-point position $(0.1 \mathrm{bits})$. The dynamic balance between modularity and small-worldedness was once again captured in the extracted activations (Fig.8(C)), with opposing directions of correlation in the trial-to-trial fluctuations for $G E$ and $Q$ that were all significant $(p<0.05)$. T3 activations were most strongly related to integrative changes ( $R=0.75)$, once again likely reflecting the differing muscular involvement required at end-point position while $\mathrm{T} 2$ demonstrated the strongest association with trial-to-trial changes in modularity $(R=-0.83)$. 
bioRxiv preprint doi: https://doi.org/10.1101/2021.10.15.464450; this version posted October 20, 2021. The copyright holder for this preprint (which was not certified by peer review) is the author/funder, who has granted bioRxiv a license to display the preprint in perpetuity. It is made available under aCC-BY-NC 4.0 International license.

(A)

$\mathrm{T} 1$
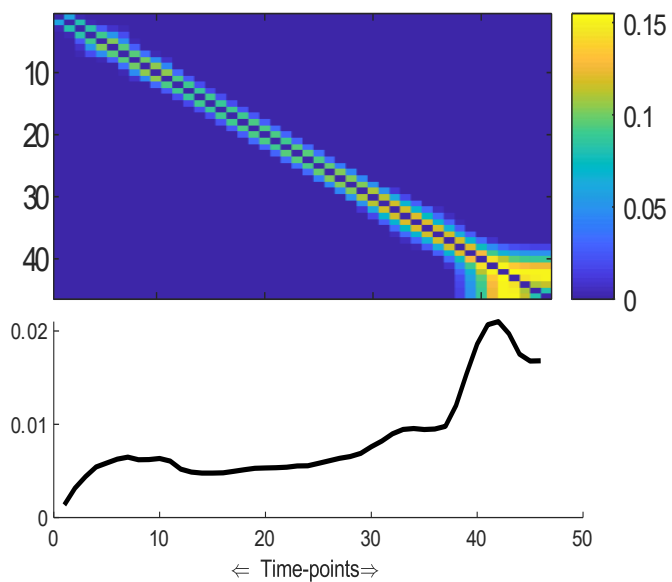

$\mathrm{T} 2$
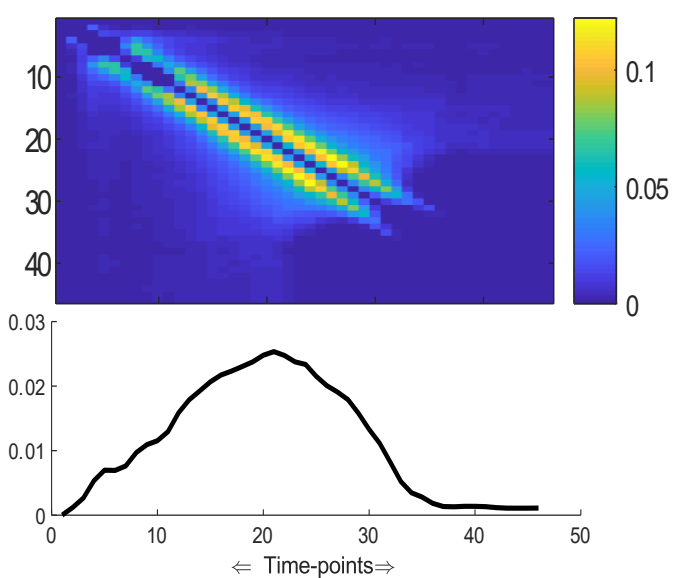

T3
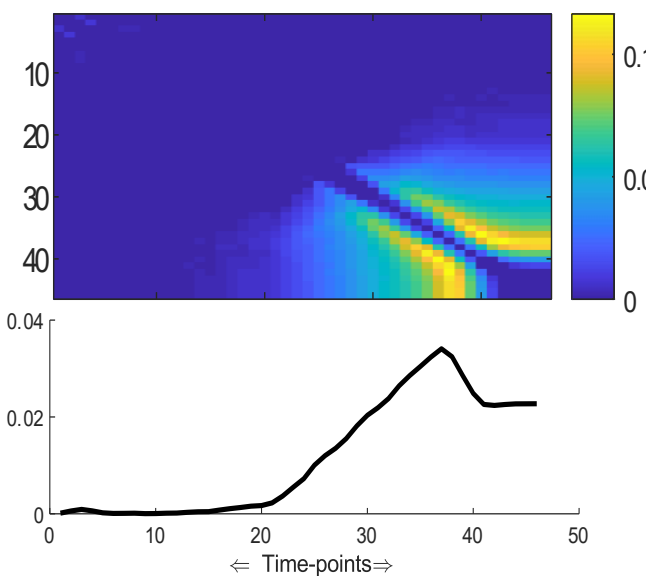

(B)
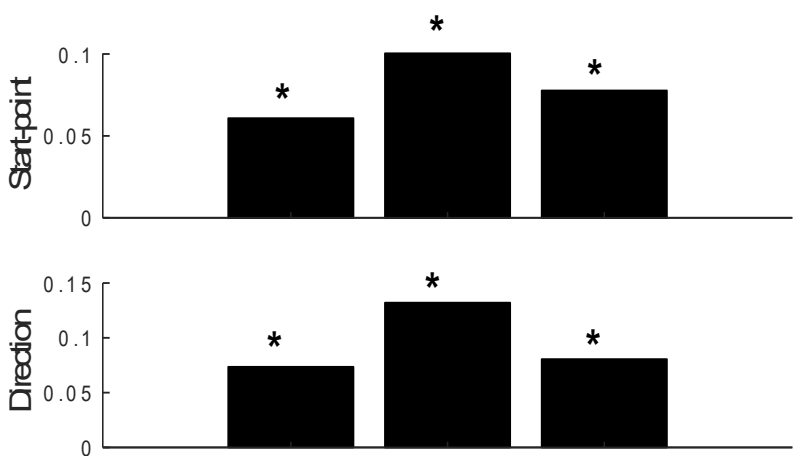

*

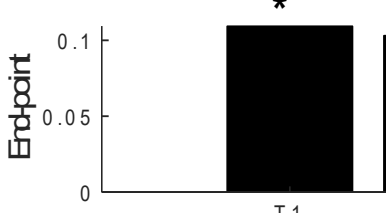

T 1
*

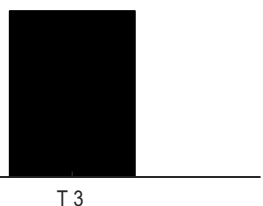

(C)
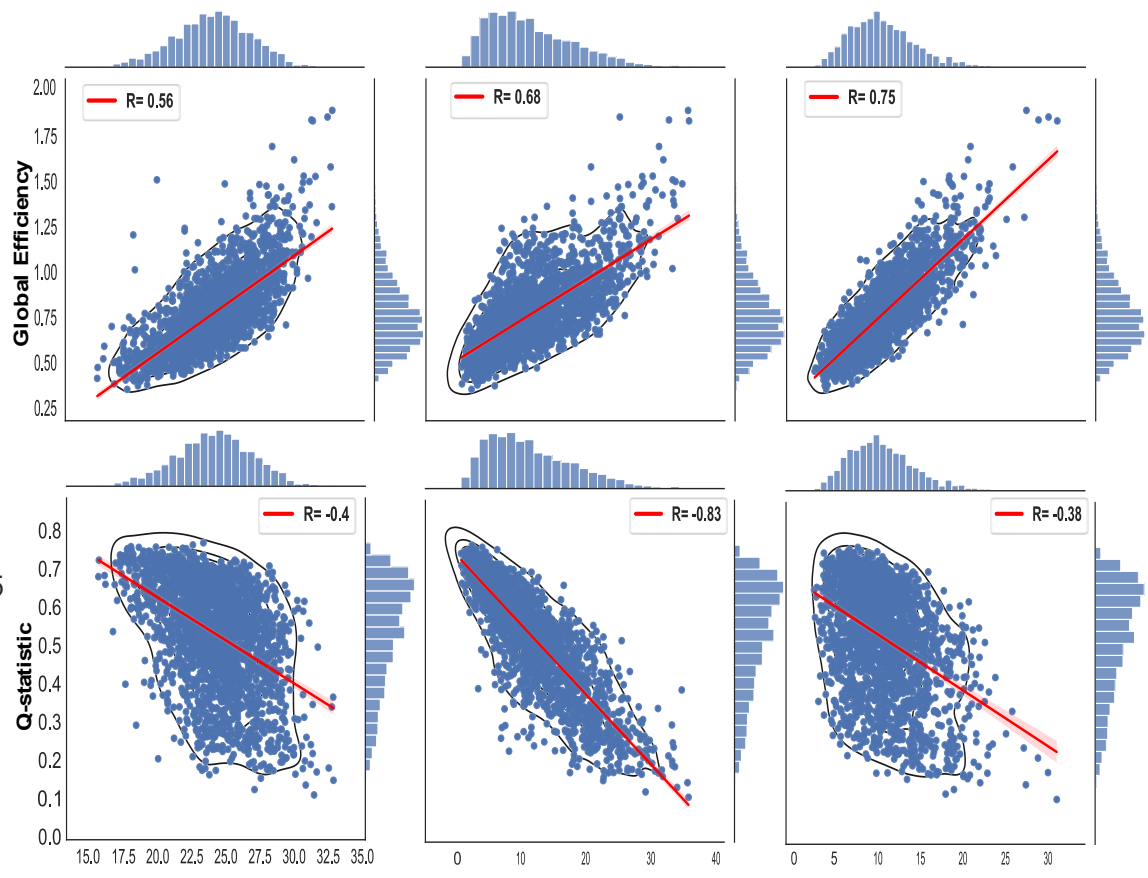

$\mathrm{T} 1$
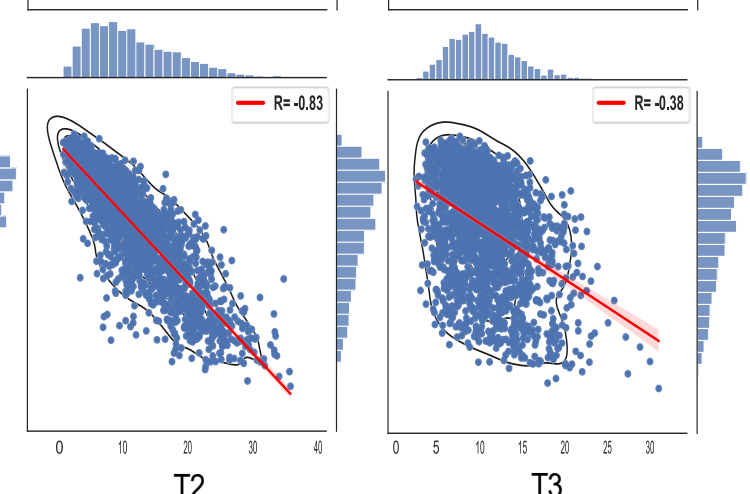

T3

Fig. 8: (A) Temporal synergies extracted from an example participant in dataset 2. Three communities were identified in the multiplex network and extracted using PNMF. The line plot below represents the average values within each column of the adjacency matrix. (B) The task-encoded information is presented in bits. Significant information $(p<0.05)$ is indicated with *. (C) The noise correlations between each temporal synergy activation and Global Efficiency/Q-statistic. 
bioRxiv preprint doi: https://doi.org/10.1101/2021.10.15.464450; this version posted October 20, 2021. The copyright holder for this preprint (which was not certified by peer review) is the author/funder, who has granted bioRxiv a license to display the preprint in perpetuity. It is made available under aCC-BY-NC 4.0 International license.

Space-Time synergy model
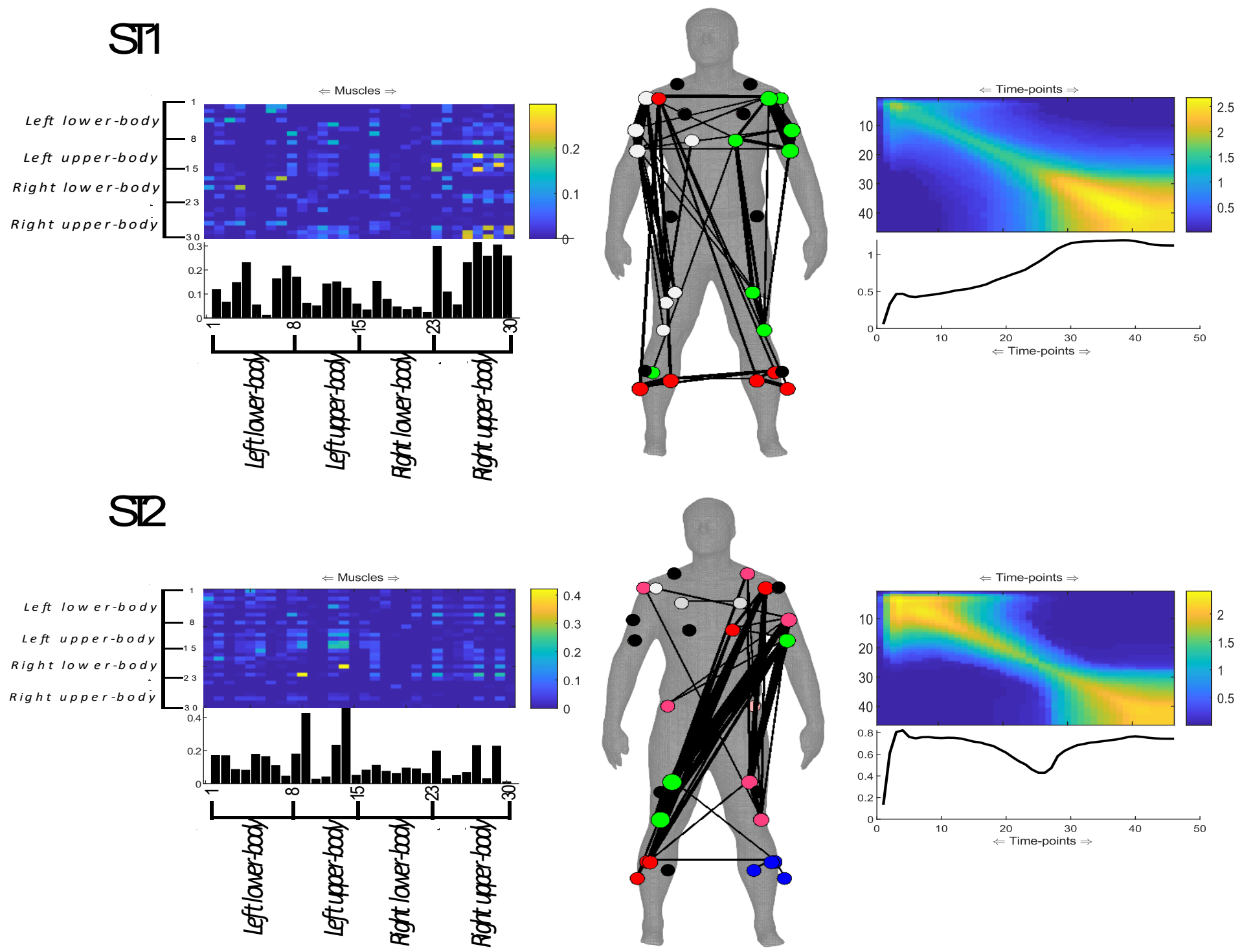

ST3
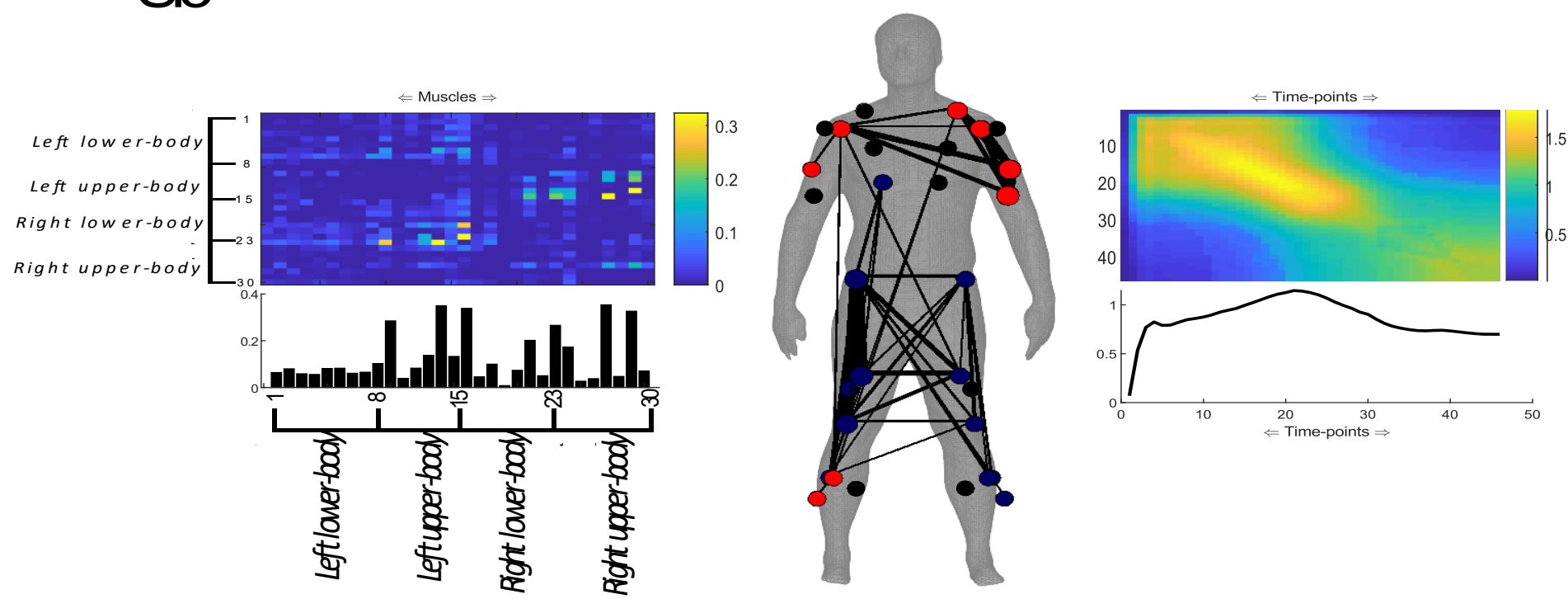

Fig.9: The Space-Time synergies extracted from the example participant in dataset 2. Three communities were identified and extracted using PNMF. Spatial and temporal synergies correspond on a 1:1 basis here as presented across rows. The spatial synergies are organ ised so that rows 1-8: left lower-limb, rows 9-15: left upper-body, rows 16-23: right lower-limbs and rows 24-30: right upper-body. The bar/ line plots below represents the average values within each column of the corresponding adjacency matrix. The connection strengths, submodular structure and involvement of nodes are indicated by the human body model via the edge widths, node colour and size respectively [50]. Submodular structure was identified using the conventional Louvain algorithm on the synergy matrices [40]. Unconnected nodes are in black. 
bioRxiv preprint doi: https://doi.org/10.1101/2021.10.15.464450; this version posted October 20, 2021. The copyright holder for this preprint (which was not certified by peer review) is the author/funder, who has granted bioRxiv a license to display the preprint in perpetuity. It is made available under aCC-BY-NC 4.0 International license.

We then sought to generalise the space-time synergy model results from dataset 1 to the example participant in dataset 2 (Fig.9). The findings were successfully replicated in a more complex dataset of whole-body reaching where a model rank of three was found. ST1 here appears to capture the postural stabilisation exhibited predominantly in the upper-body but supported by the femoral muscles at movement onset and increasingly so at movement termination. ST2 is characterised by a phasic activation at movement initiation with a critical muscle (anterior deltoid) identified at the reaching shoulder supported by dependencies among the lower-limb muscles of both sides that counteract the induced shifts in centre-of-pressure. Following movement initiation, ST3 comes into a greater level of dependency between timepoints 12-28 approximately, accompanied by significant connections between the gluteals and among the right side lower-limb. This synergy then subsides back to its original activation level near movement termination, where the new body position is supported by the sustained activation in ST1. A similar level of dependency is found within the left upper-body and the right anterior deltoid in ST2 with this synergy reflecting the increased demands induced proximally in order to counteract the right arm during the transition to a new position. A complex submodular structure was found across all three synergies, as exemplified on the human body models where three, five and two submodules were identified by a secondary community detection of ST1, ST2 and ST3 respectively. The right anterior deltoid and tibialis musculature (red), right arm and femoral musculature (white) and left arm and femoral musculature (green) comprised ST1 submodules. ST2 consisted of strong connections between the left upperand right-lower body (green, red \& wine) and a small cluster among left tibialis musculature (blue). ST3 comprised strong connections among the right lower-limb but also with the left leg (navy) along with a cluster of muscles across the upper-body that contained a long-range connection with the right soleus and anterior tibialis (red).

\section{Consistency of synergies (Dataset 2)}

Following synergy extraction, we determined the consistency of identified spatial, temporal and space-time synergies across participants during whole-body reaching movements using a structural similarity index. Starting with the spatial synergy model, 3.4 (range=3-4) synergies were representative of the five participants in dataset 2 with the Q-statistic ranging from 0.997-0.998 and the threshold value 0.26 bits on average. The similarity index revealed a moderate level of correlation ( $R=0.56 \pm 0.19)$. Supplementary materials Fig.5(A-C) illustrates the average spatial synergies we extracted along with their respective task attribute dependencies and correlation with network properties. Despite this reduced similarity, the synergies are readily interpretable in their underlying functionality, likely indicating that the shape of activations rather than functionality was dissimilar across participants. For instance, $\mathrm{S} 1$ here consisted of dependencies within both the upper- and lower-body related to normal-stance stability and S2 is related to whole-body postural stability during reaching movements. As the greatest dependencies in S3 lie within the right upper-body, this synergy is representative of the activity in the reaching arm. These observations are supported by the task-encoded information. For instance for $\mathrm{S} 2$, the average task information was noticeably higher for startpoint height ( 0.26 bits) and Up-down direction ( 0.28 bits) over other synergies, likely reflecting the differing postural adjustments required as the reaching arm is either raised up against gravity or down closer to a resting position. The trade-off between GE and $Q$ captured in the activation coefficients was replicated across participants. Interestingly, fluctuations in $S 3$ activations were most sensitive to changes in $G E(R=0.69)$ and $Q(R=-0.57)$ across the remaining participants.

We then conducted this analysis on the temporal synergies of dataset 2 participants, producing a mean of 3.4 synergies (range $=3-4$ ) across participants that exhibited a high degree of concordance $(R=0.91 \pm 0.57)$. This high level of concordance was accompanied by significant variability which we posit reflects the varying time-lags and contributions of individual muscles to reaching movements across participants that wouldn't influence the overall shape of the synergy but the composition (i.e. higher/lower magnitude dependencies comparatively). The Q-statistic ranged from 0.97-0.995 while the average threshold value was 1.45 bits. In Supplementary materials Fig.6(A-C), the representative temporal synergies for this sample are presented along with mean task-attribute and noise correlations with network properties. T2 was most highly modulated by start-point ( 0.05 bits) and direction (0.07 bits). T1 and T3 shared an equivalent level of modulation by end-point ( 0.546 and 0.547 bits respectively). The fluctuations in T3 activations were most sensitive to changes in $G E(R=0.69)$ and $Q(R=-0.57)$, although all synergies demonstrated a high association across participants.

Finally, we applied the space-time model to all dataset 2 participants and their structural similarity was compared using a representative model rank of 2 (mean rank= 2.4, range=2-3). We found a satisfactory level of concordance across participants $(R=0.79 \pm 0.4)$. Supplementary materials Fig.7 illustrates these representative synergies where the Q-statistic for modularity ranged from 0.93-0.997 and the threshold values ranged from 0.295- 
bioRxiv preprint doi: https://doi.org/10.1101/2021.10.15.464450; this version posted October 20, 2021. The copyright holder for this preprint (which was not certified by peer review) is the author/funder, who has granted bioRxiv a license to display the preprint in perpetuity. It is made available under aCC-BY-NC 4.0 International license.

0.385 bits. ST1 functionally represents the deceleration and support of the reaching arm in its new position, which is reflected by the involvement of the upper- and lower-body of the opposing side. ST2 was active during the early to intermediary stages of the movement and mostly consists of couplings between lower-limb muscles, providing postural stabilisation during task execution.

\section{Discussion}

To summarise the findings presented, functionally and physiologically meaningful muscle synergies were extracted using an information-theoretic framework. Synergies were extracted in the spatial, temporal and spatiotemporal domains, each capturing unique sub-tasks and potentially representing underlying mechanisms in human point-to-point reaching generalisable across two datasets that were consistent in their projections across participants. Along with this, we showed a significant task dependence of synergy activations that closely related to the functional interpretations of the extracted synergies and significant correlations with network properties that captured the dynamic trade-off between modularity and small-worldedness. Both model-rank selection and sparsification procedures were conducted prior to dimensionality reduction in a data-driven and holistic manner that reduces the necessity for post-hoc analyses. Through the determination of dependencies on a within-trial basis between muscles, time-vectors or their combination across trials, a novel formulation of muscle synergy was implemented that we suggest can provide important and novel insights into the neural control of human movement.

Continuity with previous muscle synergy models

Previous studies have been conducted on the datasets analysed here with interesting commonalities with our results $[14,53,54]$. Functional muscle groupings by the spatial synergy model here were reflective of previous findings (e.g. Dataset 1: elbow extensors (S1), shoulder flexors (S2) and shoulder and elbow extensors (S3); Dataset 2: a single synergy (S3) for the reaching arm along with whole-body functional groupings relevant for postural stability that cannot be explained by anatomical constraints). The spatial synergies presented in the current study were, similarly to previous reports, only moderately similar across participants in their shape but robust in their functional underpinning. This low level of agreement found in dataset 2 is likely related to physical discrepancies among participants (e.g. height, muscle length, motor preferences). The concordance among temporal synergies found in the current study also replicated previous work. Notably however, a high degree of inter-subject variability was reported here. As the temporal synergies consisted of dependencies between timepoint vector pairings across muscles, we posit that in contrast to the shape differences among spatial synergies, the overall shape of the temporal synergies was consistent but their composition was not. The varying time-lags of individual muscles is likely to have contributed to this difference in composition [55,56], leading to comparatively higher/lower magnitude dependency at certain timepoint pairs across participants. This demonstrates the capacity for the GCMI framework to effectively capture the idiosyncrasies of individual participants' movement patterns. To exemplify this point further, following a functional similarity analysis of temporal synergies it was noted in [54] that a number of the four synergies identified could be merged into fewer clusters. In the current study, these synergies were indeed merged into a readily identifiable biphasic pattern accompanied by an underlying tonic synergy, highlighting the functional relevancy of the synergies extracted here. The ability of GCMI to capture complex, nonlinear associations was advantageous in the merging of functionally synonymous phasic synergies.

In terms of task dependence, significant shared information was observed in the activation coefficients of spatial and temporal synergies with various task attributes. An interesting difference in the task modulation of muscle synergies extracted here to that presented in previous work is that the synergies identified here convey information for several movement phases. This may reflect the unique formulation introduced by the GCMI computations where early activations provide relevant task information about future states and vice-versa within the same vector in the input matrices. This allows for individual synergies to provide a more holistic insight into the task performed, representing synergies collectively as a single motor representation that unfolds across space and time. There was also some continuity with previous research. For example in dataset 2, whole-body reaching spatial synergies associated with postural stability were consistently modulated by task attributes that could be said to influence the participants medio-lateral or anterior-posterior centre-of-pressure (e.g. End-point height, Up-Down direction) as formerly observed [54]. The transient but also decelerative temporal synergy in dataset 2 (T2) was highly modulated by reaching direction, a replication of previous work also. The task modulation of space-time synergies has been reserved for investigation in future work. 
bioRxiv preprint doi: https://doi.org/10.1101/2021.10.15.464450; this version posted October 20, 2021. The copyright holder for this preprint (which was not certified by peer review) is the author/funder, who has granted bioRxiv a license to display the preprint in perpetuity. It is made available under aCC-BY-NC 4.0 International license.

\section{Novel insights from the GCMI framework}

The GCMI framework presents a number of advantageous qualities that have provided novel insights in the current study. In past work, the separation of tonic and phasic synergies has not been straightforward and commonly involved the subtraction of a linear ramp from EMG waveforms [57,58]. Within the GCMI framework, these distinct temporal synergy types are both isolated within the same extraction procedure (e.g. T1 of fig.5 and ST1 in fig.6), leading to a data-driven approach that relaxes the assumption of linearity. In relating the mechanisms underlying the postural control of movement in the oculomotor system and the human arm, [59] elucidated a plausible mechanism for subcortical postural control. The integration of cortically generated movement commands by a separate, subcortical postural controller was initially supported in primates and found to be generalizable to healthy human and corticallyimpaired populations where this dependence persisted on a within-trial basis. In the current study, tonic synergies were identified in reaching tasks on a within-trial basis only that consistently presented a pattern of activation indicative of the aforementioned findings. More specifically, a low-to-moderate level of dependency was found throughout T1 (dataset 1) until a sharp spike in dependency was found consistently across participants at the last timepoint pairings, potentially representing the integration of the preceding movement activations in the holding response. Nonetheless, when generalised to the more complex dataset 2 the characteristic tonic synergy shape was not sufficiently identified. The GCMI framework allows for the relaxation of rigid temporal alignments and therefore future investigations on higher resolution EMG data may provide greater clarity in synergy output with findings that may run in parallel to recent innovations in time-warped tensor decompositions [60].

The ability for a self-organising system to re-organise its elemental variables from trial-to-trial to complete a given task is an important attribute of the synergy concept [23]. In the development of this framework, careful consideration for important network properties characteristic of biological systems was made throughout the analysis. We chose to focus on two opposing but essential network properties, fractal modularity and small-worldedness, due to their functional implications and focus in the relevant literature $[34,36,42-44,61]$. The preservation of these important network properties in the extracted synergies was confirmed through the identification of significant noise correlations in the underlying activation coefficients with trial-to-trial fluctuations in modularity and global efficiency. The persistent opposing directions and strength of these associations with GE and Q are both reflections of this tradeoff and the sensitivity of the extracted synergies to trial-to-trial fluctuations at the network level respectively. These findings are also in line with the current understanding of the phenomena of adaptive breakdowns in modularity in complex systems and findings relating gain modulation to shifts in neural network structure [61,62]. Furthermore, the maintenance of fractal modularity in the output of the GCMI framework is exemplified by the consistent presence of submodules within the extracted synergies. It is likely that many of these submodules were defined by biomechanical constraints, however, a significant number of long-range connections clearly transcended this constraint (e.g. the right anterior deltoid and lower-limb musculature in the human body models of dataset 2). Therefore synergies extracted through the GCMI framework may be useful for investigating the unique contribution of both biomechanical and neural constraints on movement.

The identification of movement phase commencement and cessation has been frequently cited in the literature as difficult, especially during fast-paced movements due to overlapping muscle activations [58,63]. The orthogonality introduced by PNMF here effectively removes this limitation by minimising the overlap between temporal synergies. In both datasets, a novel bi-phasic pattern supported by an underlying tonic synergy was revealed. This bi-phasic pattern is reflective of the bang-bang control policy investigated recently that was found to explain the characteristic tri-phasic activation of reaching movements. The findings presented here support the hypothesis of an intermittent rather than continuous control scheme in human motor control and implicate this control policy as a fundamental mechanism underlying human motor control $[64,65]$.

Muscle synergy analysis is known to be scale-dependent, in that the number and spread of muscles analysed across the body influences the synergy output, typically by increasing model-rank with increasing dataset complexity [38]. Instead, the proposed framework identified a reduced number of spatial and temporal synergies that were invariant to dataset complexity. Within the sensory system the multiplex encoding of information across multiple timescales is well-known [66], allowing for an increased coding capacity and the apparent continuous stream of perception. This multiplexity has more recently been identified in the encoding of task information from low-dimensional dynamics in a motor cortical neural population and put forward as a structural mechanism underlying cognition and selforganisation more generally [67-69]. Other recent research that has modelled muscle synergies as a multiplex network only identified a single module representing a global co-activation during gait in various coordination modes [20]. This global co-activation may have masked more discrete but important mechanisms that could not be uncovered by 
bioRxiv preprint doi: https://doi.org/10.1101/2021.10.15.464450; this version posted October 20, 2021. The copyright holder for this preprint (which was not certified by peer review) is the author/funder, who has granted bioRxiv a license to display the preprint in perpetuity. It is made available under aCC-BY-NC 4.0 International license.

simpler methodologies. In the current study, the multiplexity of human motor control was exhibited through the parsing of several community structures across multiple spatial and temporal scales that demonstrated significant encoding of task information. Therefore, we suggest that the GCMI framework presents as a useful tool for investigating the emergence of complex patterns in motor behaviour.

\section{Future research directions}

A key motivation for the development of this framework was to address the limitations of linear assumptions and lack of flexibility in current muscle synergy models. The inclusion of task space variables has recently come into the spotlight in this line of research [10], as the pattern configurations found are required to be constrained by an objective function in order to be functionally relevant and transferable to robotics and prosthetic design $[10,70]$. Through the induction of non-parametric statistical tools in information and network theory, the presented formulations are now more amenable to the inclusion of task space variables while more appropriately capturing the non-linearity of the musculoskeletal system. The objective function to constrain these extracted synergies may also involve the inclusion of neural data derived elsewhere in the CNS, producing neurophysiological relevant synergies that are falsifiable [1]. Each muscle synergy model may capture distinct motor features [71], thus investigating their functional underpinning following the inclusion of these constraints may be fruitful. Information theory more generally has found great use in the analysis of complex systems, for instance in identifying synergistic and redundant interactions in the CNS, the integration of cross-modal informational dynamics and providing a framework for understanding causal emergence [72-74]. Quantifying informational dynamics among muscles in space and time may allow for the identification of task-relevant and irrelevant spaces, providing a bridge between neurophysiological and purely computational frameworks $[75,76]$.

\section{References}

[1] Cheung VCK, Seki K. Approaches to revealing the neural basis of muscle synergies: a review and a critique. J Neurophysiol 2021;125:1580-97.

[2] Berret B, Delis I, Gaveau J, Jean F. Optimality and modularity in human movement: from optimal control to muscle synergies. InBiomechanics of Anthropomorphic Systems 2019 (pp. 105-133). Springer, Cham.

[3] Bernstein N. The co-ordination and regulation of movements. The co-ordination and regulation of movements. 1966.

[4] Giszter SF, Hart CB. Motor primitives and synergies in spinal cord and after injury-the current state of play. Annals of the New York Academy of Sciences. 2013 Mar;1279:114.

[5] Bizzi E, Cheung VC. The neural origin of muscle synergies. Frontiers in computational neuroscience. 2013 Apr 29;7:51.

[6] Kutch JJ, Valero-Cuevas FJ. Challenges and new approaches to proving the existence of muscle synergies of neural origin. PLoS computational biology. 2012 May 3;8(5):e1002434.

[7] Overduin SA, d'Avella A, Carmena JM, Bizzi E. Microstimulation activates a handful of muscle synergies. Neuron. 2012 Dec 20;76(6):1071-7.

[8] Kargo WJ, Giszter SF. Rapid correction of aimed movements by summation of force-field primitives. Journal of Neuroscience. 2000 Jan 1;20(1):409-26.

[9] Cheung VC, Cheung BM, Zhang JH, Chan ZY, Ha SC, Chen CY, Cheung RT. Plasticity of muscle synergies through fractionation and merging during development and training of human runners. Nature communications. 2020 Aug 31;11(1):1-5.

[10] Alessandro C, Delis I, Nori F, Panzeri S, Berret B. Muscle synergies in neuroscience and robotics: from inputspace to task-space perspectives. Frontiers in computational neuroscience. 2013 Apr 19;7:43.

[11] Tresch MC, Saltiel P, Bizzi E. The construction of movement by the spinal cord. Nature neuroscience. 1999 Feb;2(2):162-7.

[12] Dominici N, Ivanenko YP, Cappellini G, d'Avella A, Mondì V, Cicchese M, Fabiano A, Silei T, Di Paolo A, Giannini C, Poppele RE. Locomotor primitives in newborn babies and their development. Science. 2011 Nov 18;334(6058):997-9.

[13] d'Avella A, Lacquaniti F. Control of reaching movements by muscle synergy combinations. Frontiers in computational neuroscience. 2013 Apr 19;7:42.

[14] Delis I, Panzeri S, Pozzo T, Berret B. A unifying model of concurrent spatial and temporal modularity in muscle activity. Journal of neurophysiology. 2014 Feb 1;111(3):675-93.

[15] Nazifi MM, Yoon HU, Beschorner K, Hur P. Shared and task-specific muscle synergies during normal walking and slipping. Frontiers in human neuroscience. 2017 Feb 6;11:40.

[16] Clark DJ, Ting LH, Zajac FE, Neptune RR, Kautz SA. Merging of healthy motor modules predicts reduced locomotor performance and muscle coordination complexity post-stroke. Journal of neurophysiology. 2010

Feb;103(2):844-57. 
bioRxiv preprint doi: https://doi.org/10.1101/2021.10.15.464450; this version posted October $20,2021$. The copyright holder for this preprint (which was not certified by peer review) is the author/funder, who has granted bioRxiv a license to display the preprint in perpetuity. It is made available under aCC-BY-NC 4.0 International license.

[17] Takiyama K, Yokoyama H, Kaneko N, Nakazawa K. Speed-dependent and mode-dependent modulations of spatiotemporal modules in human locomotion extracted via tensor decomposition. Scientific reports. 2020 Jan $20 ; 10(1): 1-5$.

[18] Ting LH, Macpherson JM. A limited set of muscle synergies for force control during a postural task. Journal of neurophysiology. 2005 Jan;93(1):609-13.

[19] Chvatal SA, Torres-Oviedo G, Safavynia SA, Ting LH. Common muscle synergies for control of center of mass and force in nonstepping and stepping postural behaviors. Journal of neurophysiology. 2011 Aug;106(2):9991015.

[20] Kerkman JN, Bekius A, Boonstra TW, Daffertshofer A, Dominici N. Muscle Synergies and Coherence Networks Reflect Different Modes of Coordination During Walking. Front Physiol 2020;11.

https://doi.org/10.3389/fphys.2020.00751.

[21] Boonstra TW, Faes L, Kerkman JN, Marinazzo D. Information decomposition of multichannel EMG to map functional interactions in the distributed motor system. Neurolmage 2019;202:116093.

https://doi.org/10.1016/j.neuroimage.2019.116093.

[22] Kerkman JN, Daffertshofer A, Gollo LL, Breakspear M, Boonstra TW. Network structure of the human musculoskeletal system shapes neural interactions on multiple time scales. Sci Adv 2018;4.

https://doi.org/10.1126/sciadv.aat0497.

[23] Latash ML. Synergy. Oxford University Press; 2008 Mar 18.

[24] Shannon CE. A mathematical theory of communication. The Bell system technical journal. 1948 Jul;27(3):379-

423.

[25] Friston KJ. Functional and effective connectivity: a review. Brain connectivity. 2011;1(1):13-36.

[26] Delis I, Ince RA, Sajda P, Wang Q. Information-theoretic characterization of the neural mechanisms of active multisensory decision making. InInternational Conference on NeuroRehabilitation 2018 Oct 16 (pp. 584-588).

Springer, Cham.

[27] Hart CB, Giszter SF. A neural basis for motor primitives in the spinal cord. Journal of Neuroscience. 2010 Jan $27 ; 30(4): 1322-36$.

[28] Ince RA, Giordano BL, Kayser C, Rousselet GA, Gross J, Schyns PG. A statistical framework for neuroimaging data analysis based on mutual information estimated via a gaussian copula. Human brain mapping. 2017 Mar;38(3):1541-73.

[29] Sklar M. Fonctions de repartition an dimensions et leurs marges. Publ. inst. statist. univ. Paris. 1959;8:229-31.

[30] Nelsen RB. An introduction to copulas. Springer Science \& Business Media; 2007.

[31] Yuan Z, Yang Z, Oja E. Projective nonnegative matrix factorization: Sparseness, orthogonality, and clustering. Neural Process. Lett. 2009:11-3.

[32] Yang Z, Oja E. Linear and nonlinear projective nonnegative matrix factorization. IEEE Transactions on Neural Networks. 2010 Mar 25;21(5):734-49.

[33] Boutsidis C, Gallopoulos E. SVD based initialization: A head start for nonnegative matrix factorization. Pattern recognition. 2008 Apr 1;41(4):1350-62.

[34] Gallos LK, Makse HA, Sigman M. A small world of weak ties provides optimal global integration of self-similar modules in functional brain networks. Proceedings of the National Academy of Sciences. 2012 Feb 21;109(8):2825-

30.

[35] Newman ME. Modularity and community structure in networks. Proceedings of the national academy of sciences. 2006 Jun 6;103(23):8577-82.

[36] Gallos L, Sigman M, Makse H. The conundrum of functional brain networks: small-world efficiency or fractal modularity. Frontiers in physiology. 2012 May 7;3:123.

[37] Bordier C, Nicolini C, Bifone A. Graph analysis and modularity of brain functional connectivity networks:

searching for the optimal threshold. Frontiers in neuroscience. 2017 Aug 3;11:441.

[38] Turpin NA, Uriac S, Dalleau G. How to improve the muscle synergy analysis methodology?. European Journal of Applied Physiology. 2021 Jan 26:1-7.

[39] Mucha PJ, Richardson T, Macon K, Porter MA, Onnela JP. Community structure in time-dependent, multiscale, and multiplex networks. science. 2010 May 14;328(5980):876-8.

[40] Blondel VD, Guillaume JL, Lambiotte R, Lefebvre E. Fast unfolding of communities in large networks. Journal of statistical mechanics: theory and experiment. 2008 Oct 9;2008(10):P10008.

[41] Lucas G. S. Jeub, Marya Bazzi, Inderjit S. Jutla, and Peter J. Mucha, "A generalized Louvain method for community detection implemented in MATLAB," https://github.com/GenLouvain/GenLouvain (2011-2019).

[42] De Pasquale F, Della Penna S, Sporns O, Romani GL, Corbetta M. A dynamic core network and global efficiency in the resting human brain. Cerebral Cortex. 2016 Oct 1;26(10):4015-33.

[43] Meunier D, Lambiotte R, Bullmore ET. Modular and hierarchically modular organization of brain networks. Frontiers in neuroscience. 2010 Dec 8;4:200.

[44] Bassett DS, Wymbs NF, Porter MA, Mucha PJ, Carlson JM, Grafton ST. Dynamic reconfiguration of human brain networks during learning. Proceedings of the National Academy of Sciences. 2011 May 3;108(18):7641-6.

[45] Stanley ML, Simpson SL, Dagenbach D, Lyday RG, Burdette JH, Laurienti PJ. Changes in brain network efficiency and working memory performance in aging. PLoS One. 2015 Apr 13;10(4):e0123950. 
bioRxiv preprint doi: https://doi.org/10.1101/2021.10.15,464450; this version posted October 20, 2021. The copyright holder for this preprint (which was not certified by peer review) is the author/funder, who has granted bioRxiv a license to display the preprint in perpetuity. It is made available under aCC-BY-NC 4.0 International license.

[46] Hasson CJ, Gelina O, Woo G. Neural control adaptation to motor noise manipulation. Frontiers in human neuroscience. 2016 Mar 1;10:59.

[47] Vergotte G, Perrey S, Muthuraman M, Janaqi S, Torre K. Concurrent changes of brain functional connectivity and motor variability when adapting to task constraints. Frontiers in physiology. 2018 Jul 10;9:909.

[48] Rubinov M, Sporns O. Complex network measures of brain connectivity: uses and interpretations. Neuroimage. 2010 Sep 1;52(3):1059-69.

[49] Delis I, Berret B, Pozzo T, Panzeri S. A methodology for assessing the effect of correlations among muscle synergy activations on task-discriminating information. Frontiers in computational neuroscience. 2013 May 13;7:54.

[50] Makarov SN, Noetscher GM, Nazarian A. Low-frequency electromagnetic modeling for electrical and biological systems using MATLAB. John Wiley \& Sons; 2015 Jun 22.

[51] Hilt PM, Delis I, Pozzo T, Berret B. Space-by-time modular decomposition effectively describes whole-body muscle activity during upright reaching in various directions. Frontiers in computational neuroscience. $2018 \mathrm{Apr}$ 3;12:20.

[52] Delis I, Berret B, Pozzo T, Panzeri S. Quantitative evaluation of muscle synergy models: a single-trial task decoding approach. Frontiers in computational neuroscience. 2013 Feb 26;7:8.

[53] Delis I, Panzeri S, Pozzo T, Berret B. Task-discriminative space-by-time factorization of muscle activity. Frontiers in human neuroscience. 2015 Jul 10;9:399.

[54] Delis I, Hilt PM, Pozzo T, Panzeri S, Berret B. Deciphering the functional role of spatial and temporal muscle synergies in whole-body movements. Scientific reports. 2018 May 30;8(1):1-7.

[55] d'Avella A, Saltiel P, Bizzi E. Combinations of muscle synergies in the construction of a natural motor behavior. Nature neuroscience. 2003 Mar;6(3):300-8.

[56] d'Avella A, Portone A, Fernandez L, Lacquaniti F. Control of fast-reaching movements by muscle synergy combinations. Journal of Neuroscience. 2006 Jul 26;26(30):7791-810.

[57] d'Avella A, Fernandez L, Portone A, Lacquaniti F. Modulation of phasic and tonic muscle synergies with reaching direction and speed. Journal of neurophysiology. 2008 Sep;100(3):1433-54.

[58] Flanders M. Temporal patterns of muscle activation for arm movements in three-dimensional space. Journal of Neuroscience. 1991 Sep 1;11(9):2680-93.

[59] Albert ST, Hadjiosif AM, Jang J, Zimnik AJ, Soteropoulos DS, Baker SN, Churchland MM, Krakauer JW, Shadmehr R. Postural control of arm and fingers through integration of movement commands. Elife. 2020 Feb 11;9:e52507.

[60] Williams AH. Combining tensor decomposition and time warping models for multi-neuronal spike train analysis. bioRxiv. 2020 Jan 1.

[61] Valverde S. Breakdown of modularity in complex networks. Frontiers in physiology. 2017 Jul 13;8:497.

[62] Shine JM, Aburn MJ, Breakspear M, Poldrack RA. The modulation of neural gain facilitates a transition between functional segregation and integration in the brain. Elife. 2018 Jan 29;7:e31130.

[63] Leib R, Russo M, d'Avella A, Nisky I. A bang-bang control model predicts the triphasic muscles activity during hand reaching. Journal of Neurophysiology. 2020 Jul 1;124(1):295-304.

[64] Groß J, Timmermann L, Kujala J, Dirks M, Schmitz F, Salmelin R, Schnitzler A. The neural basis of intermittent motor control in humans. Proceedings of the National Academy of Sciences. 2002 Feb 19;99(4):2299-302.

[65] Karniel A. The minimum transition hypothesis for intermittent hierarchical motor control. Frontiers in computational neuroscience. 2013 Feb 28;7:12.

[66] Panzeri S, Brunel N, Logothetis NK, Kayser C. Sensory neural codes using multiplexed temporal scales. Trends in neurosciences. 2010 Mar 1;33(3):111-20.

[67] Abbaspourazad H, Choudhury M, Wong YT, Pesaran B, Shanechi MM. Multiscale low-dimensional motor cortical state dynamics predict naturalistic reach-and-grasp behavior. Nature communications. 2021 Jan 27;12(1):1-9.

[68] Ramstead MJ, Kirchhoff MD, Constant A, Friston KJ. Multiscale integration: beyond internalism and externalism. Synthese. 2021 Jan;198(1):41-70.

[69] Friston K. The free-energy principle: a unified brain theory?. Nature reviews neuroscience. 2010 Feb;11(2):127-38.

[70] de Rugy A, Loeb G, Carroll T. Are muscle synergies useful for neural control?. Frontiers in computational neuroscience. 2013 Mar 21;7:19.

[71] Chiovetto E, Berret B, Delis I, Panzeri S, Pozzo T. Investigating reduction of dimensionality during single-joint elbow movements: a case study on muscle synergies. Frontiers in computational neuroscience. 2013 Feb 28;7:11.

[72] Gatica M, Cofré R, Mediano PA, Rosas FE, Orio P, Diez I, Swinnen SP, Cortes JM. High-order

interdependencies in the aging brain. Brain connectivity. 2021 May 28.

[73] Park H, Ince RA, Schyns PG, Thut G, Gross J. Representational interactions during audiovisual speech entrainment: Redundancy in left posterior superior temporal gyrus and synergy in left motor cortex. PLoS biology. 2018 Aug 6;16(8):e2006558.

[74] Rosas FE, Mediano PA, Jensen HJ, Seth AK, Barrett AB, Carhart-Harris RL, Bor D. Reconciling emergences: An information-theoretic approach to identify causal emergence in multivariate data. PLOS Computational Biology. 2020 Dec 21;16(12):e1008289. 
bioRxiv preprint doi: https://doi.org/10.1101/2021.10.15.464450; this version posted October 20, 2021. The copyright holder for this preprint (which was not certified by peer review) is the author/funder, who has granted bioRxiv a license to display the preprint in perpetuity. It is made available under aCC-BY-NC 4.0 International license.

[75] Scholz JP, Schöner G. The uncontrolled manifold concept: identifying control variables for a functional task. Experimental brain research. 1999 May 1;126(3):289-306.

[76] Todorov E, Jordan MI. A minimal intervention principle for coordinated movement. Advances in neural information processing systems. 2003:27-34. 


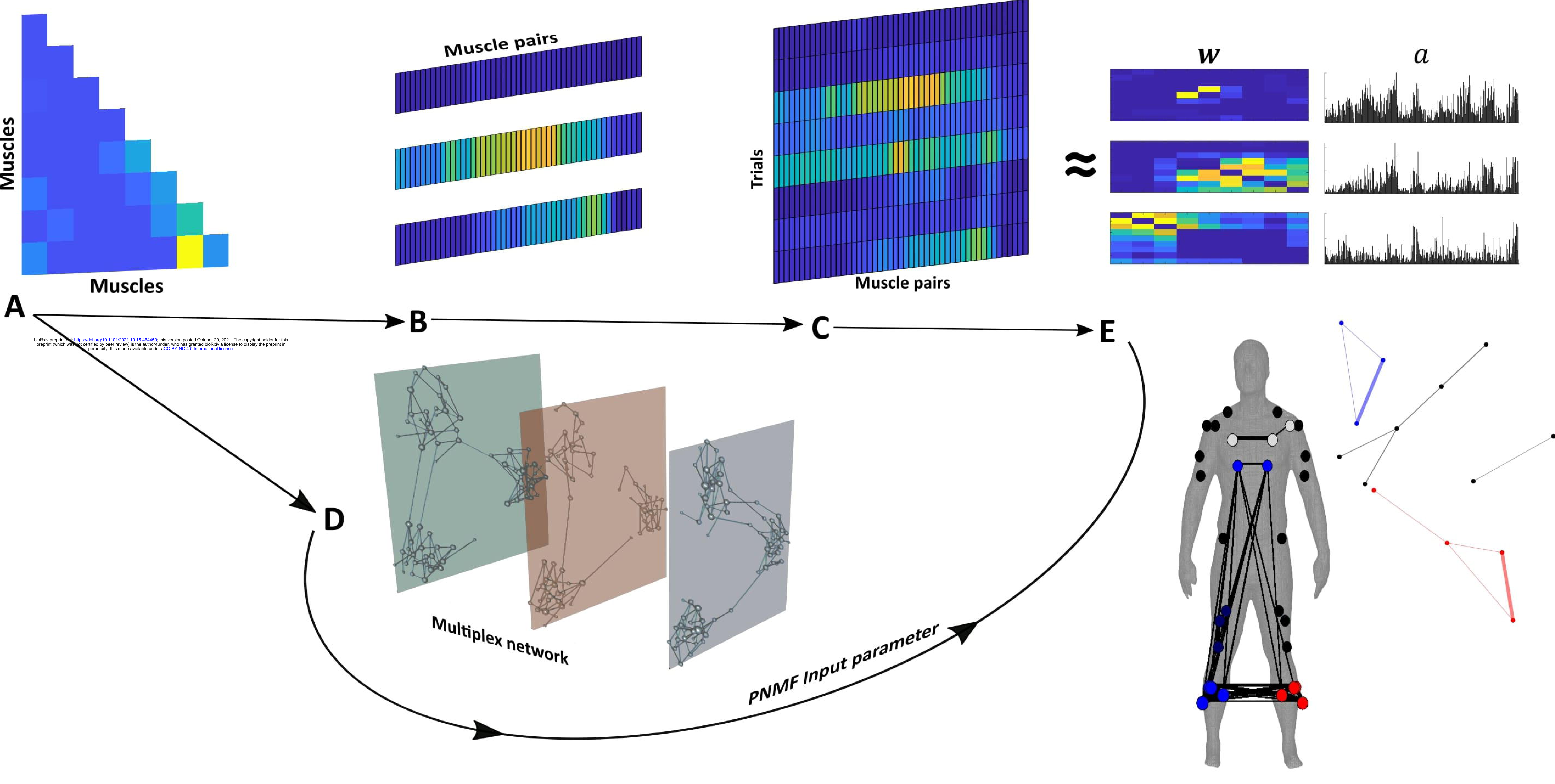




\section{(A)}
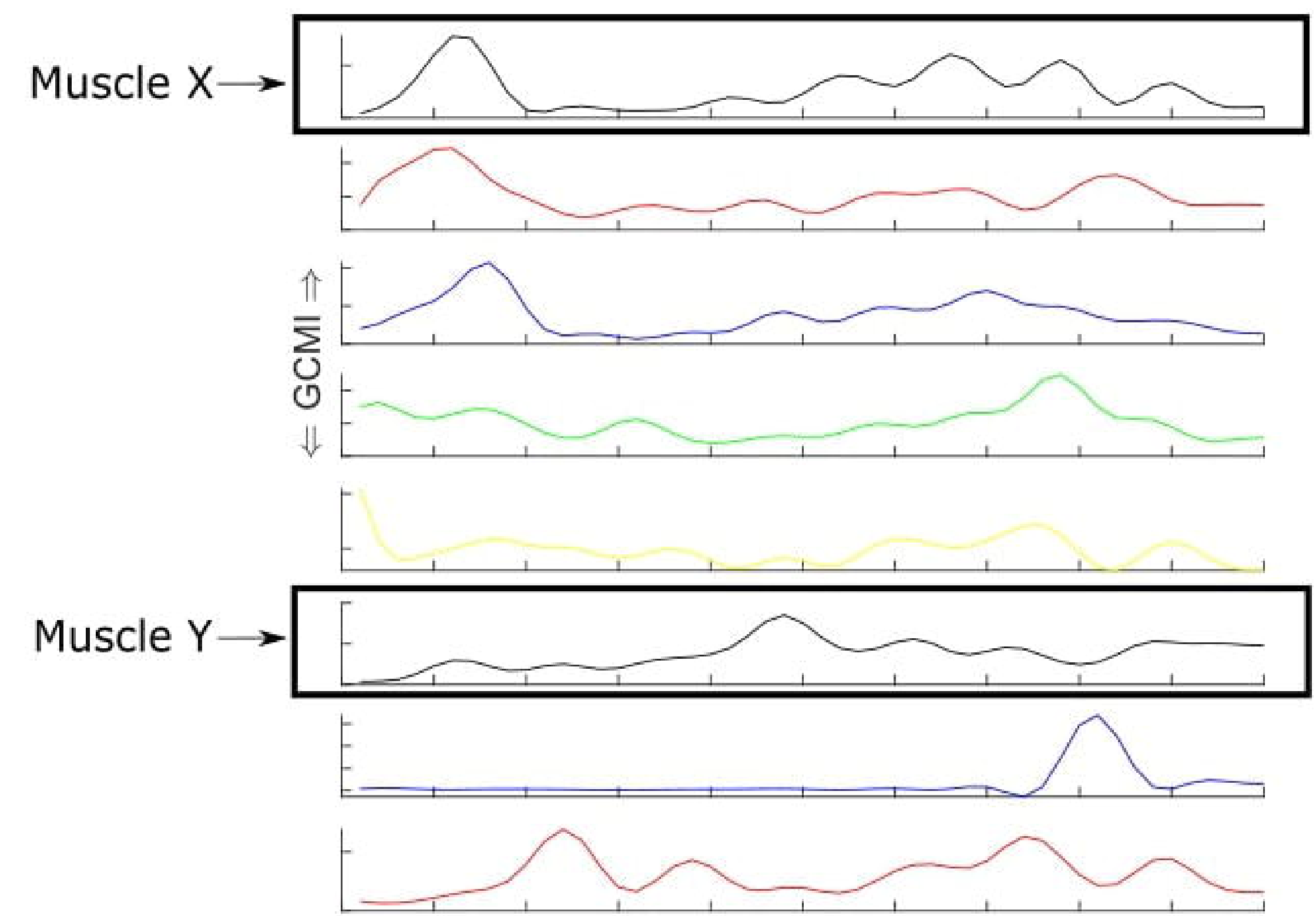

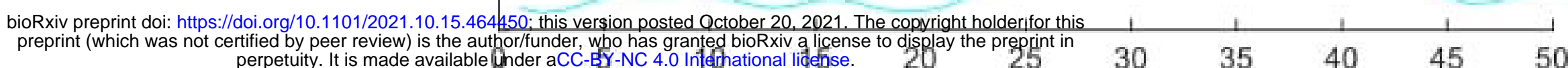

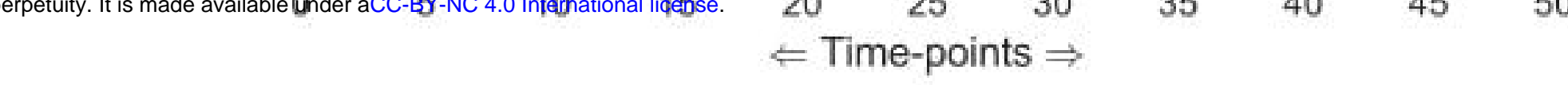

$$
\text { (C) }
$$

(B)
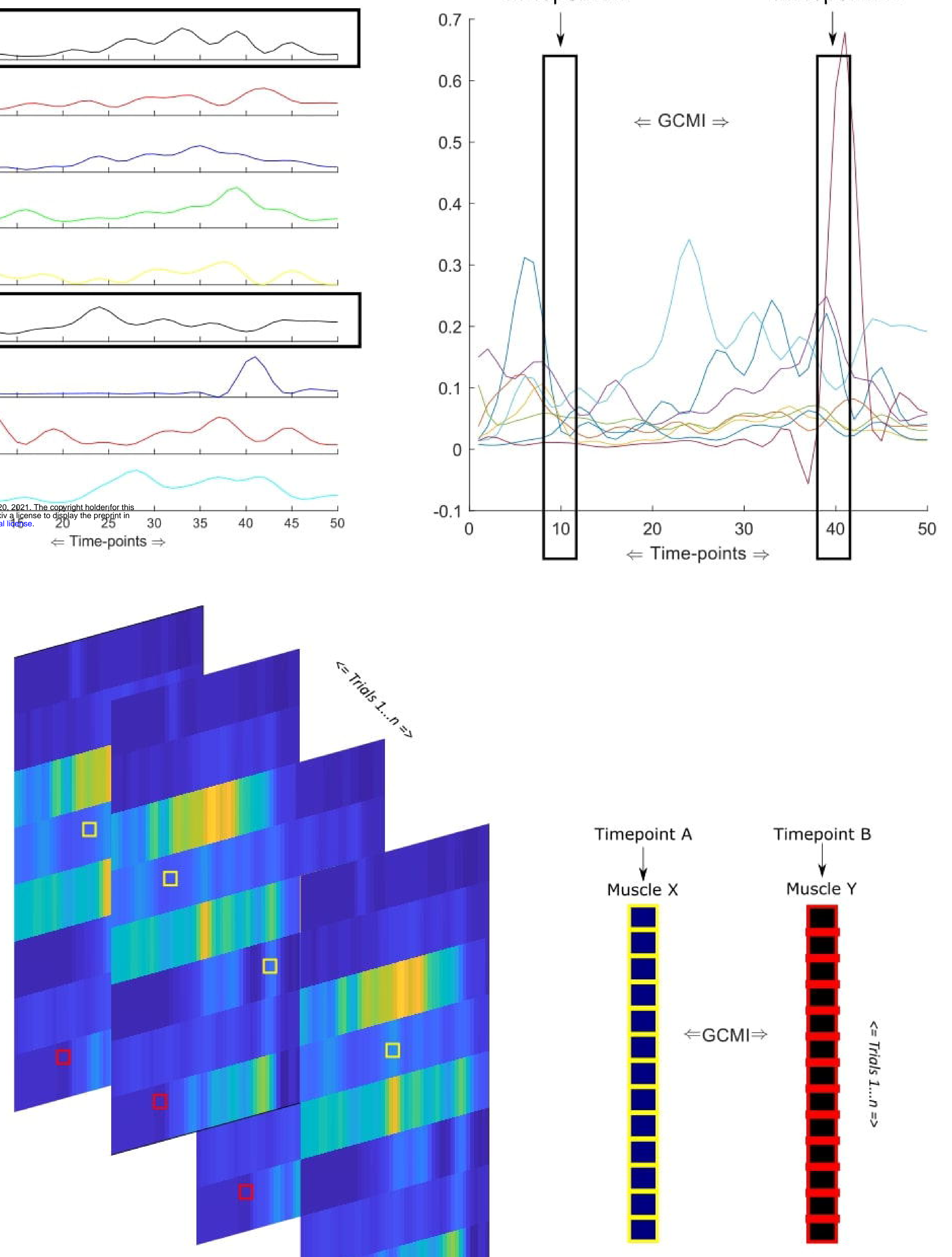


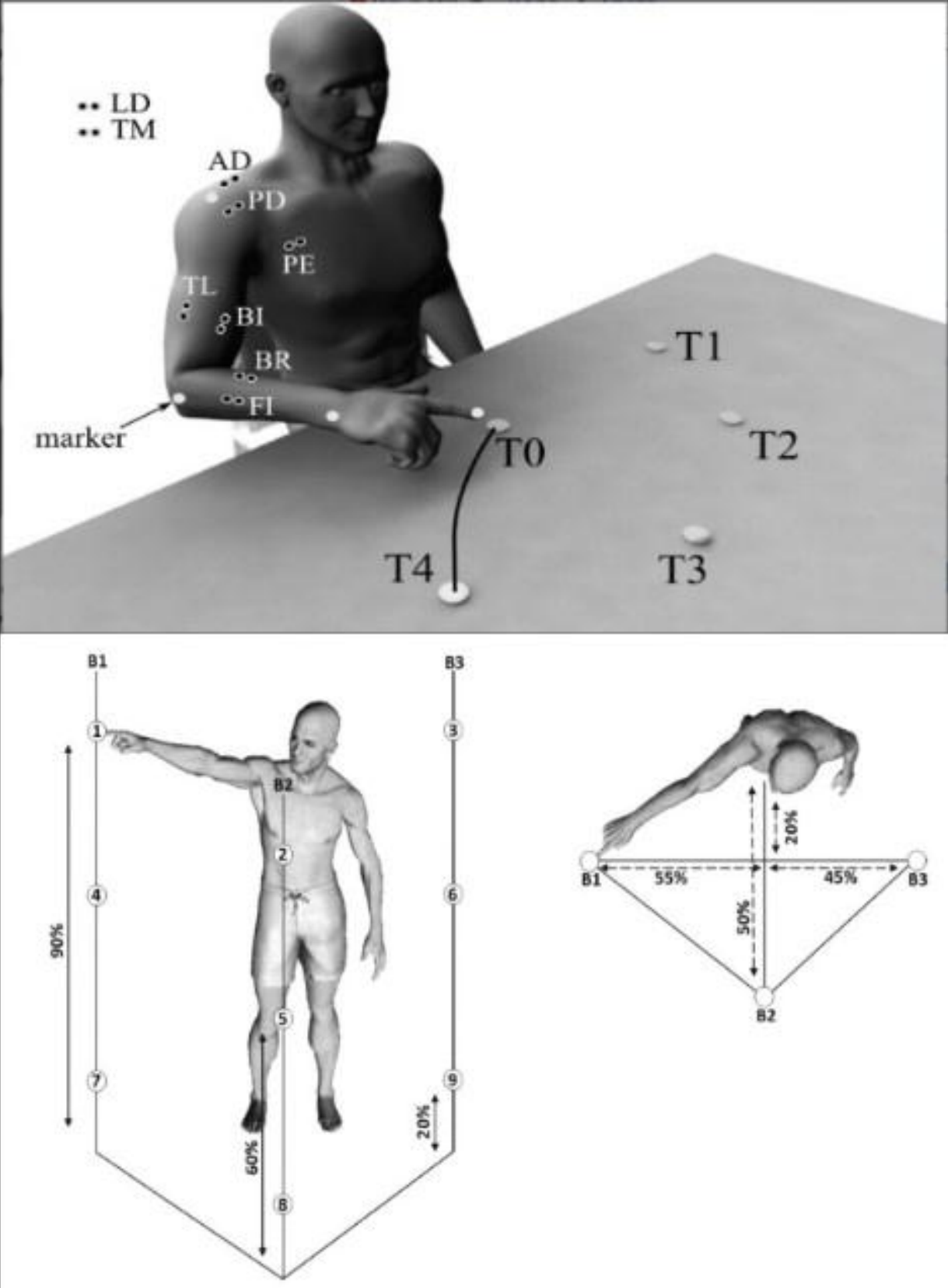


B)

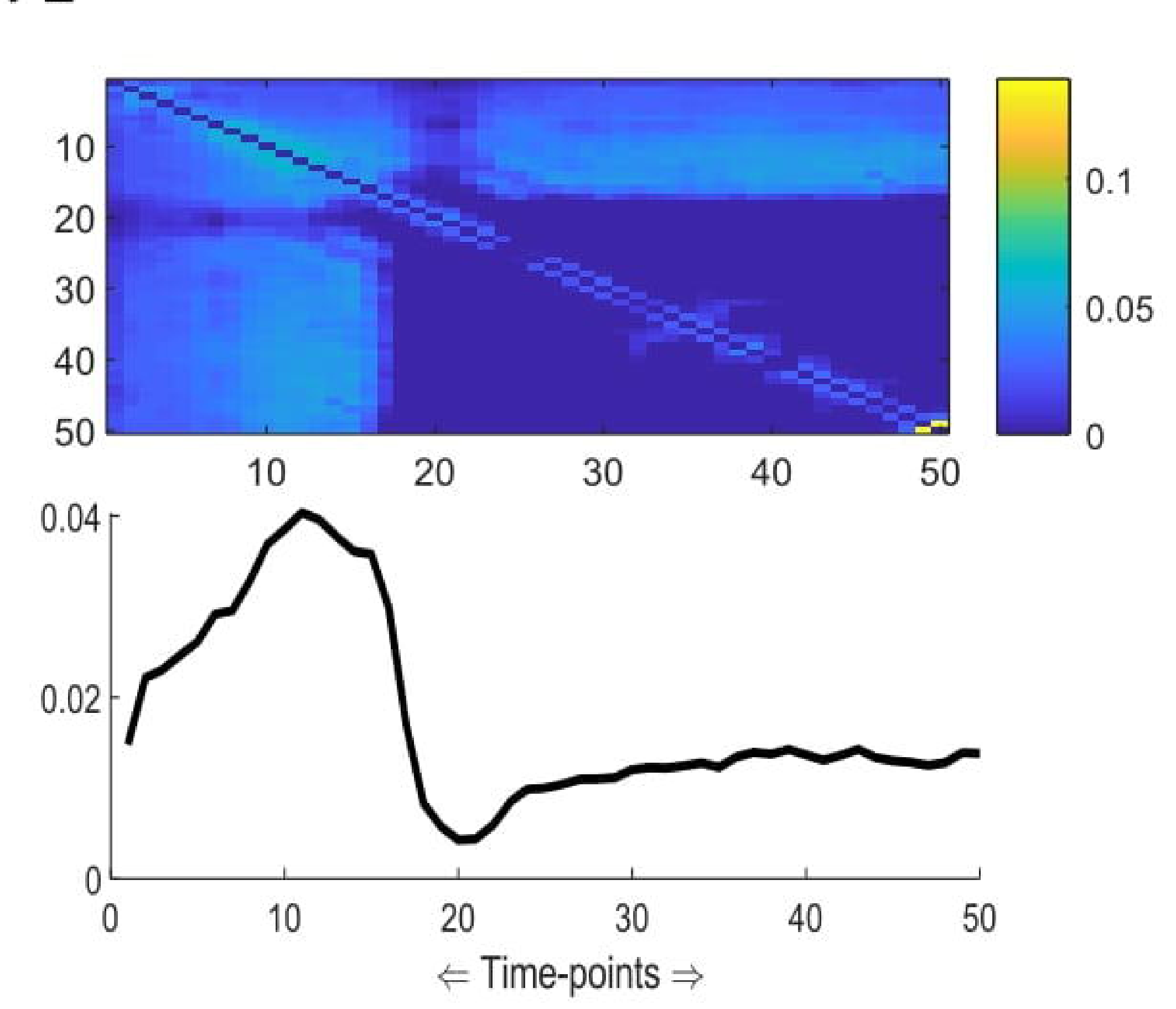

T2
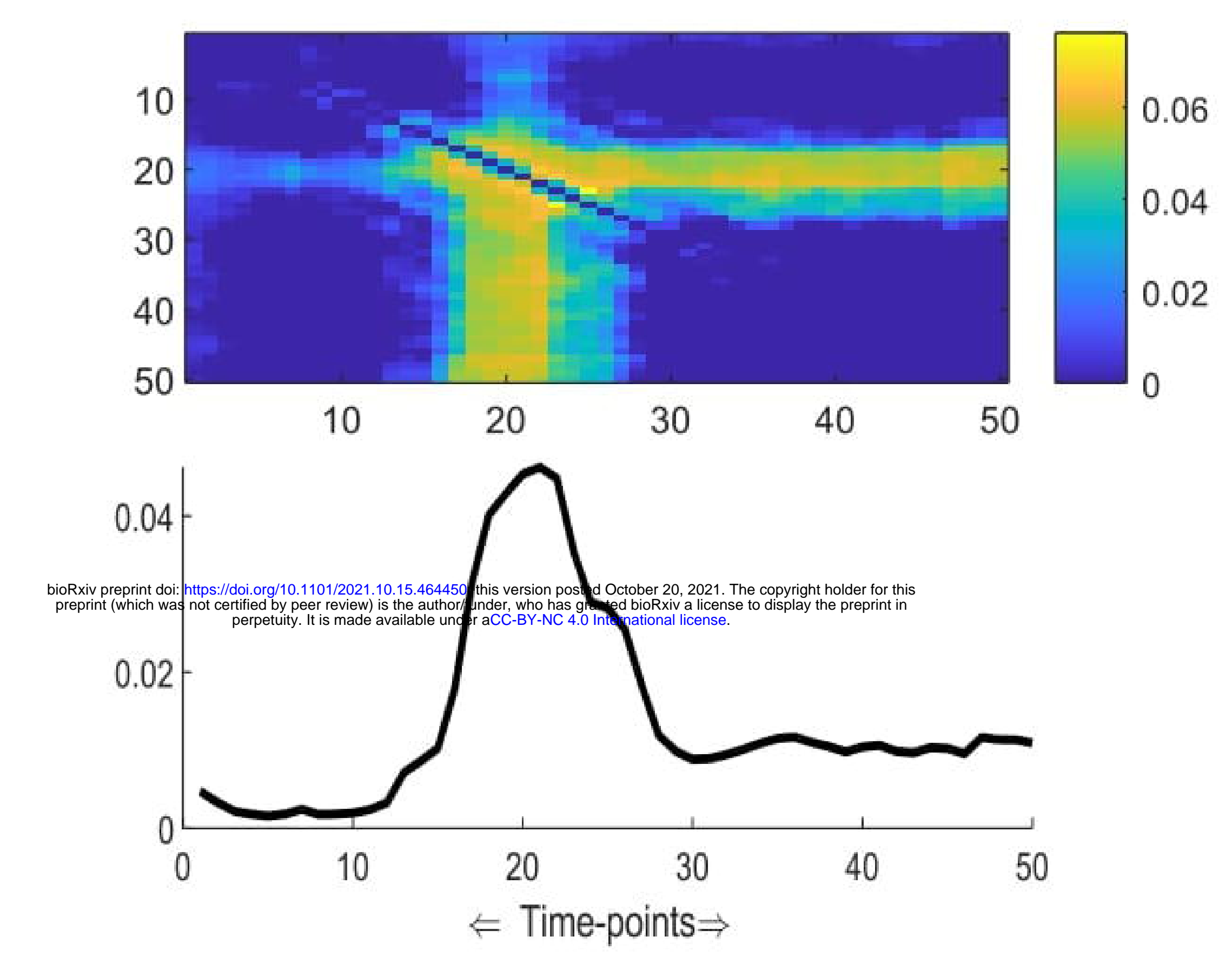

T3

$\Leftarrow$ Muscles $\Rightarrow$
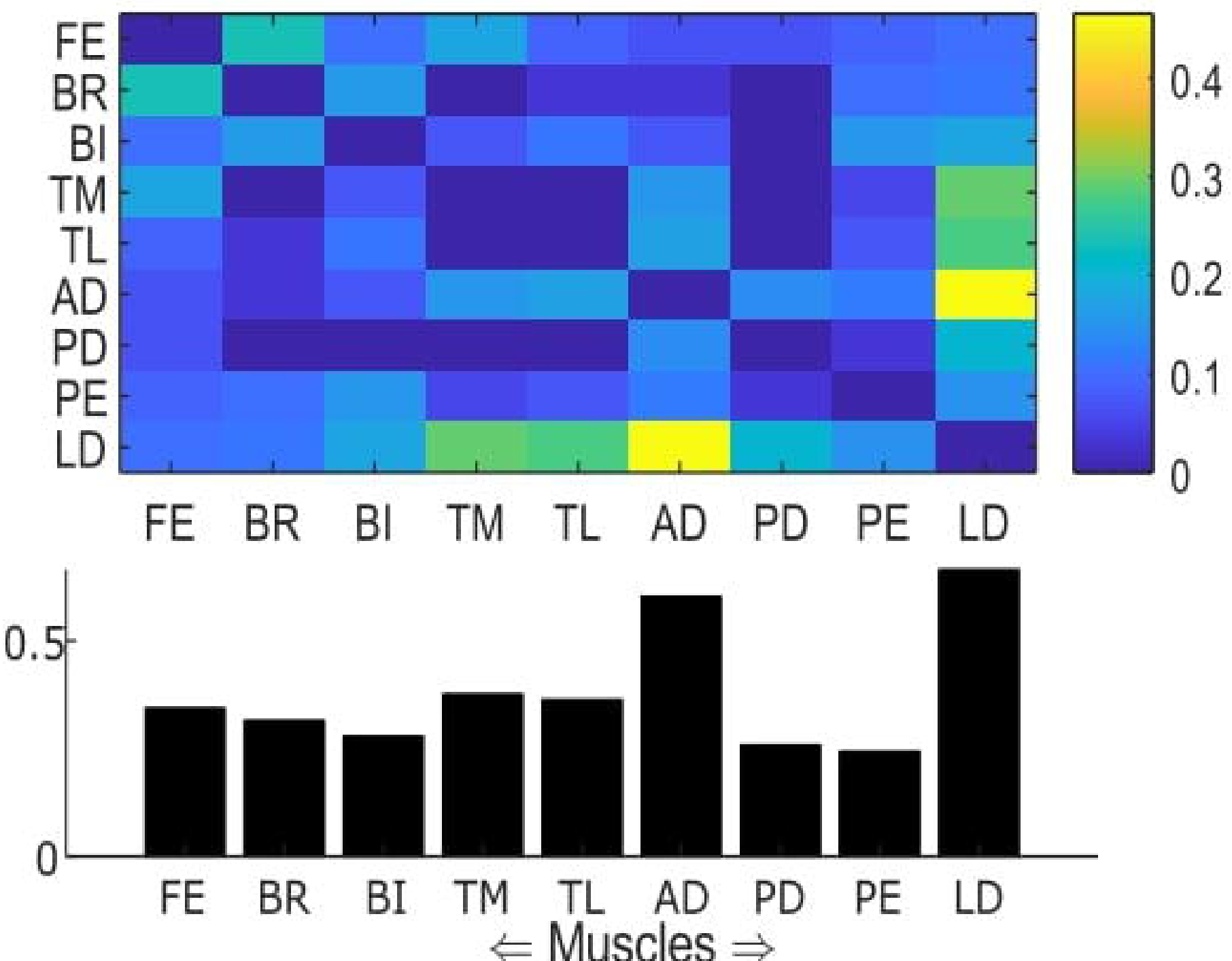

ST2

$\Leftarrow$ Muscles $\Rightarrow$

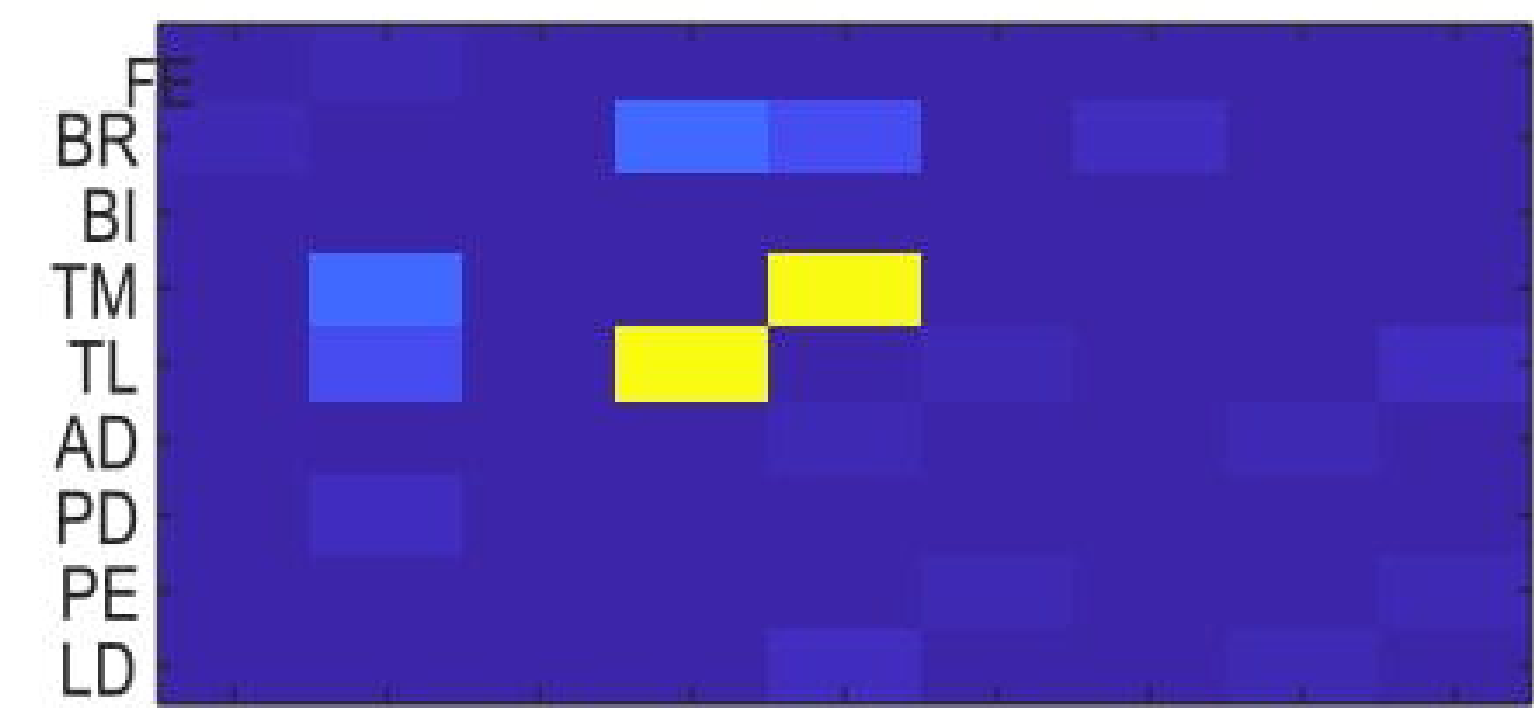

FE $\quad B R \quad B I \quad T M \quad T L \quad A D \quad P D \quad P E \quad L D$
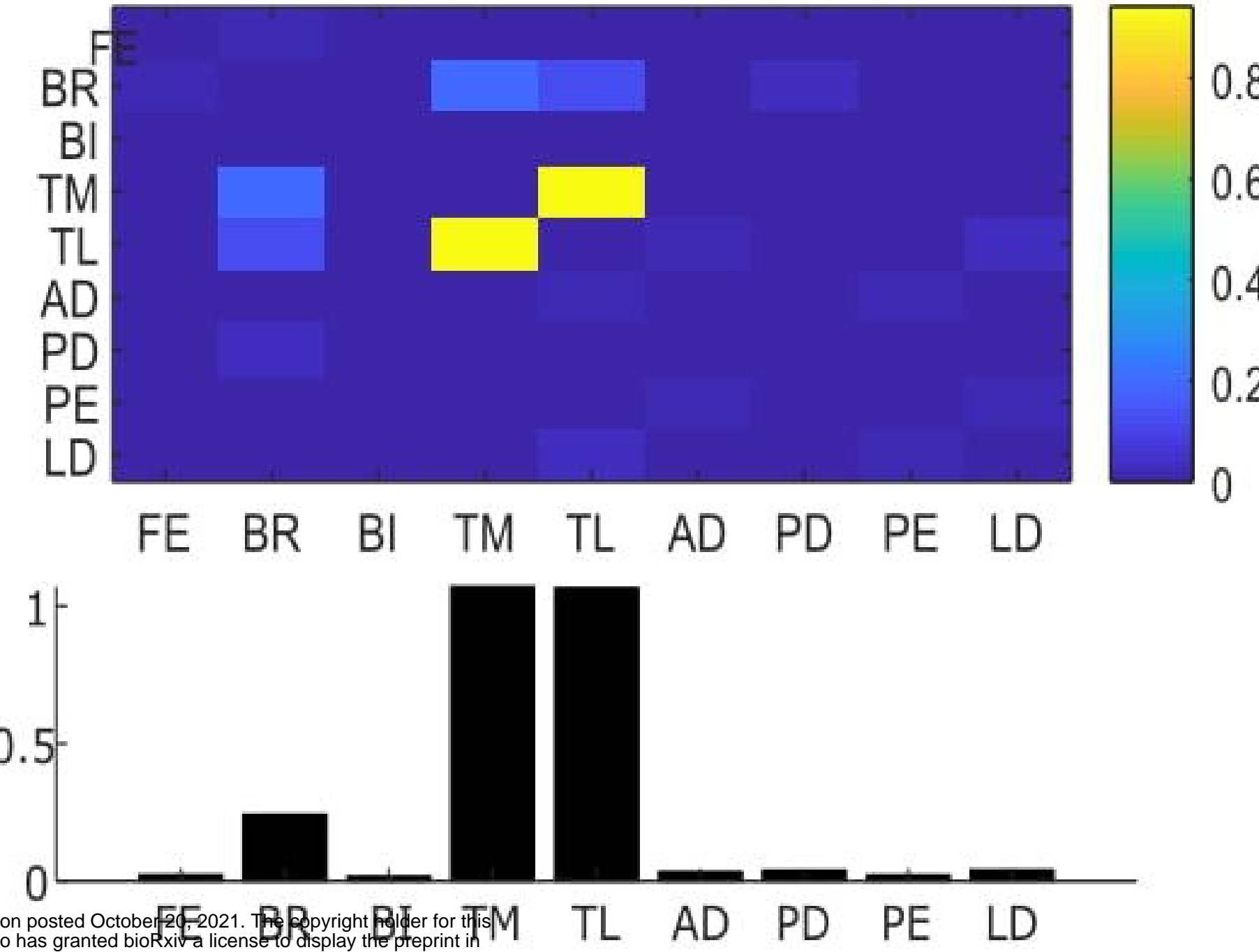

$$
\Leftarrow \text { Muscles } \Rightarrow
$$

$\Leftarrow$ Muscles $\Rightarrow$

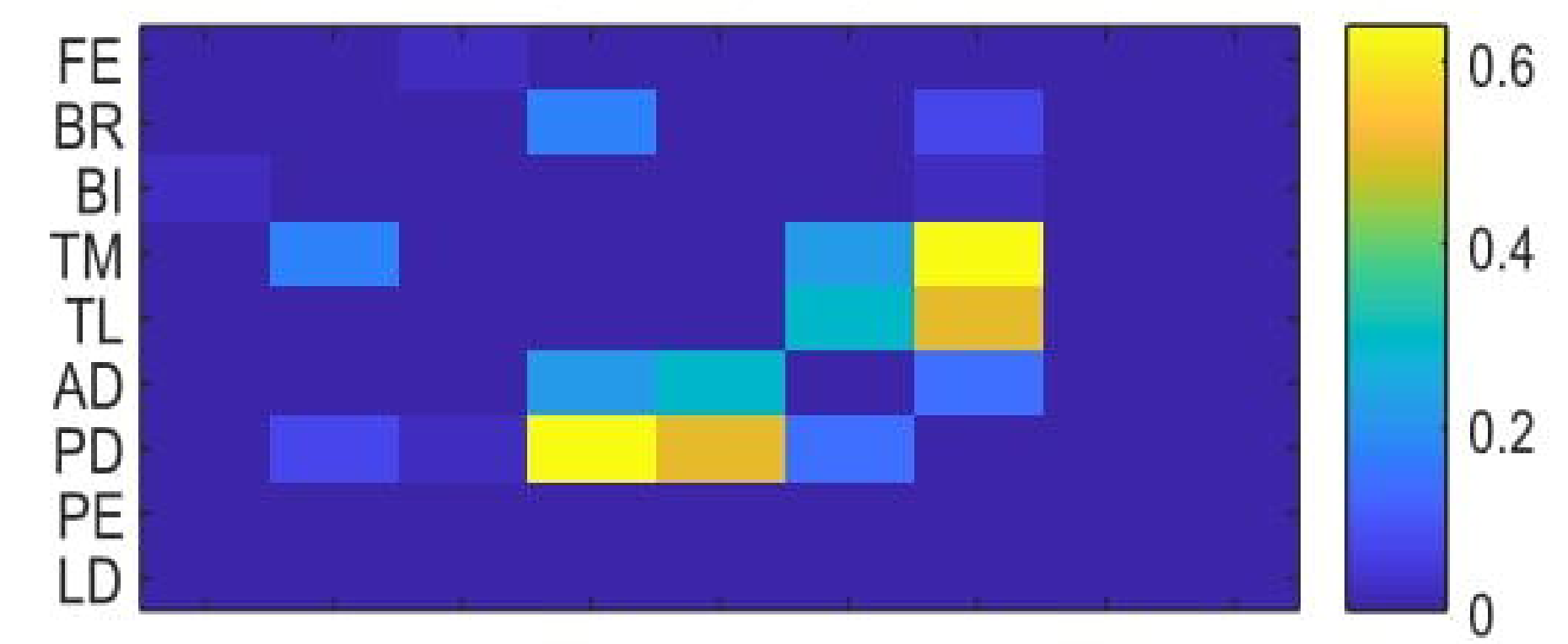

FE $\quad B R \quad B I \quad T M \quad T L \quad A D$ PD $P E \quad L D$

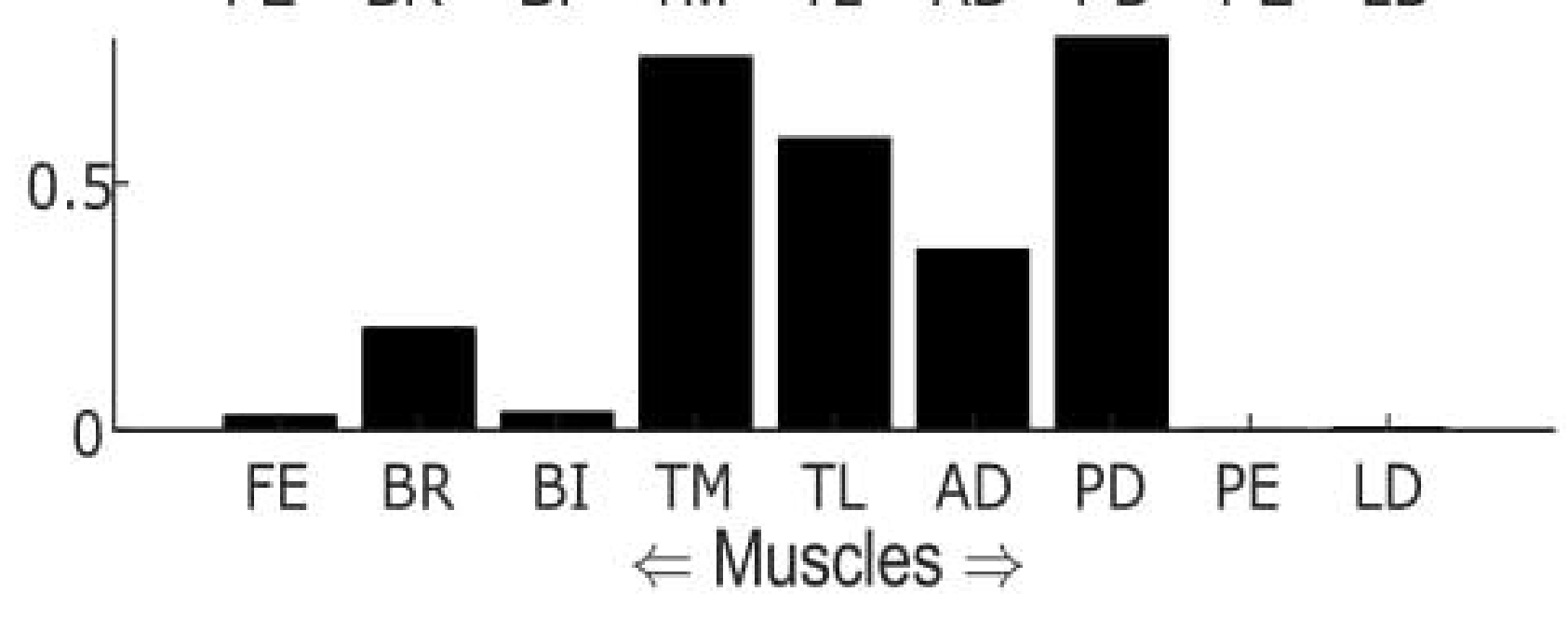

$\Leftarrow$ Time-points $\Rightarrow$
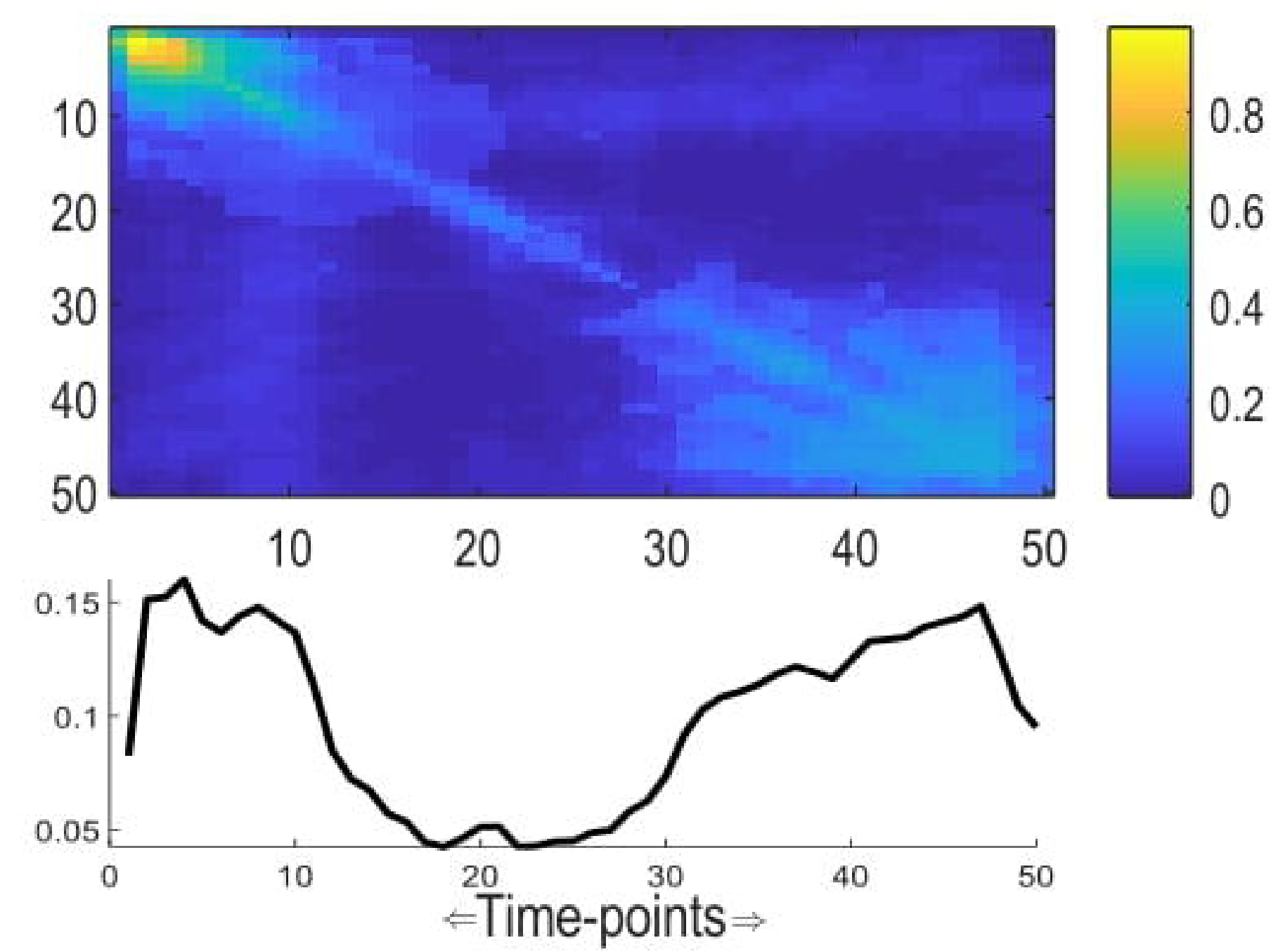

$\Leftarrow$ Time-points $\Rightarrow$
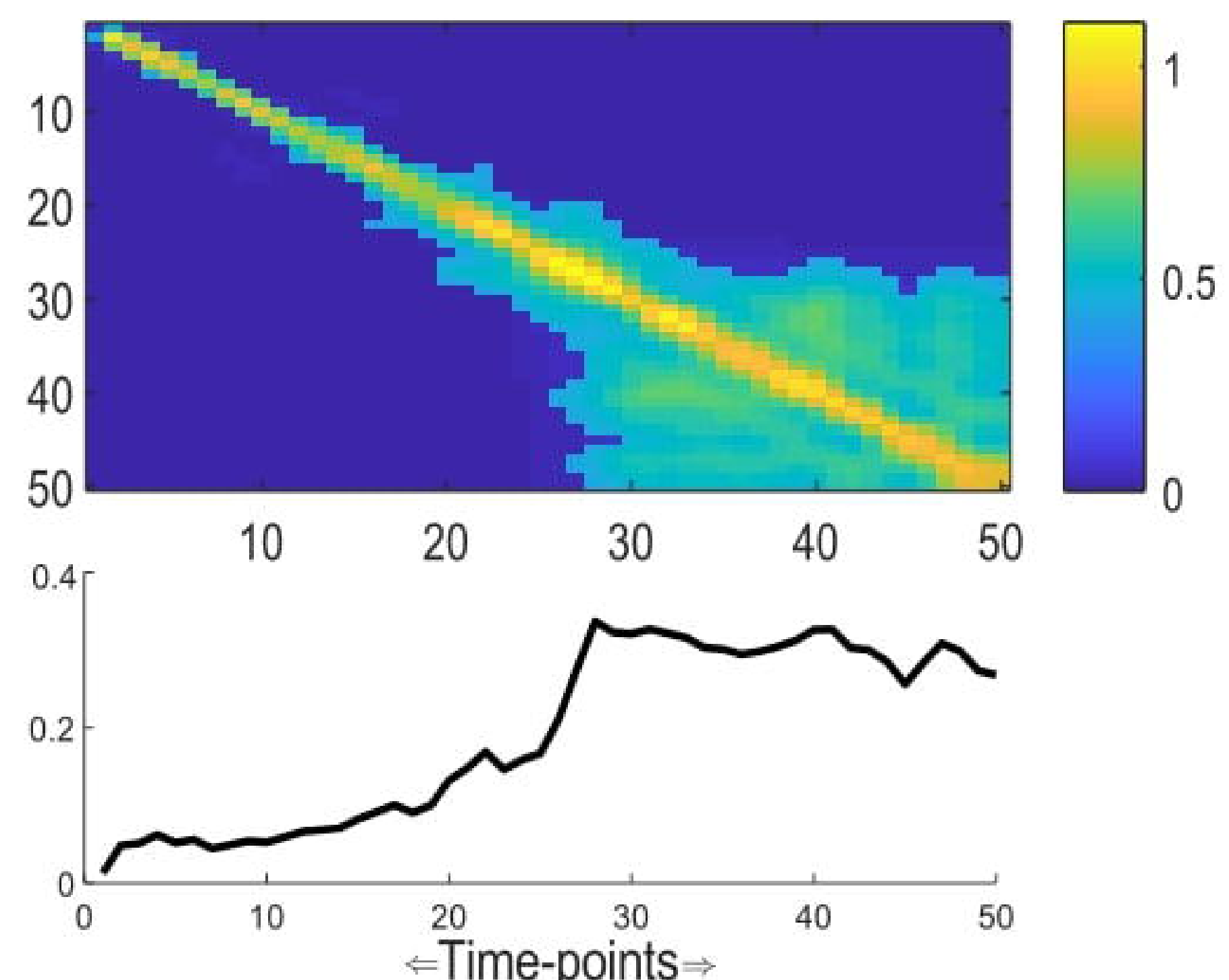

$\Leftarrow$ Time-points $\Rightarrow$
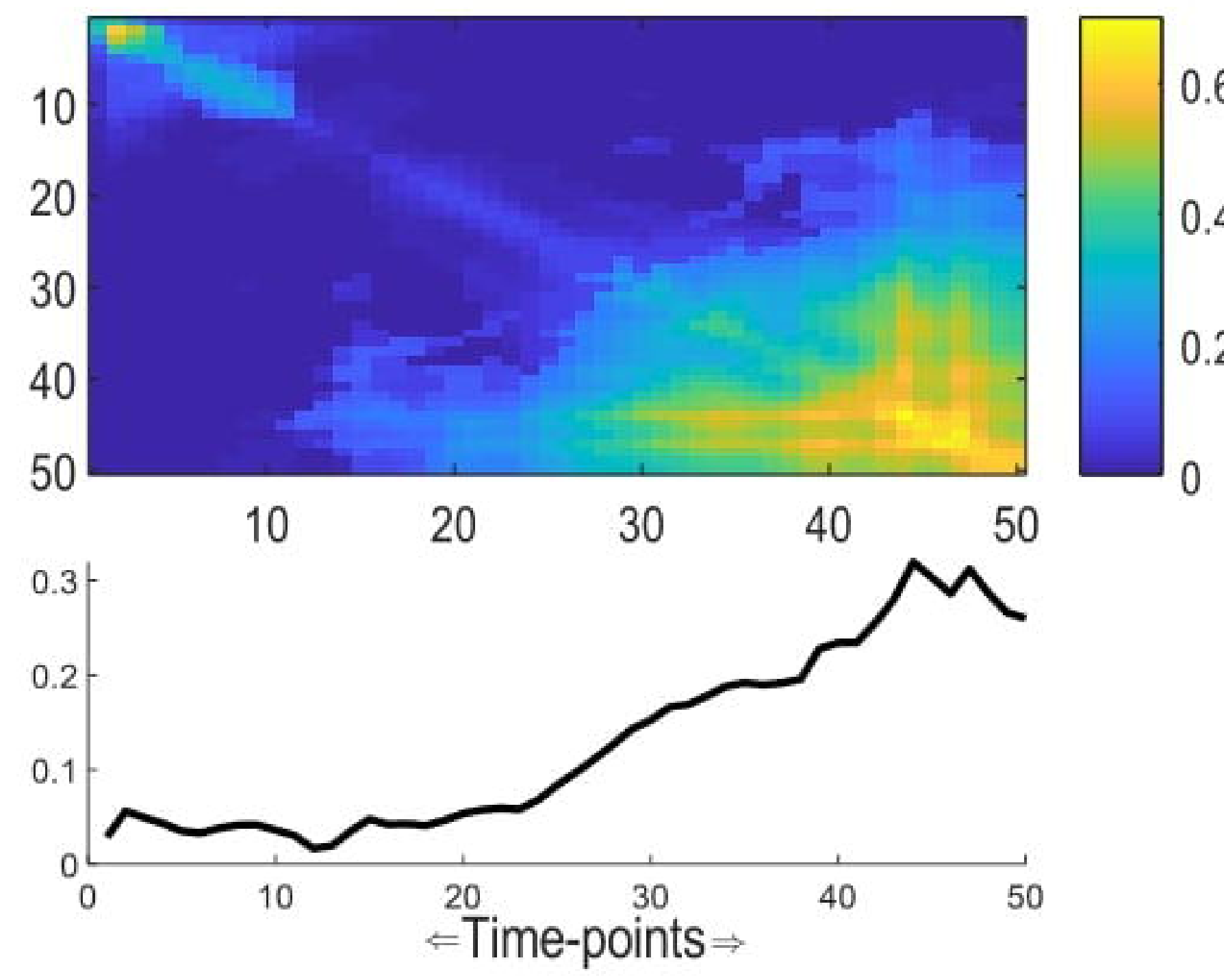
(A)

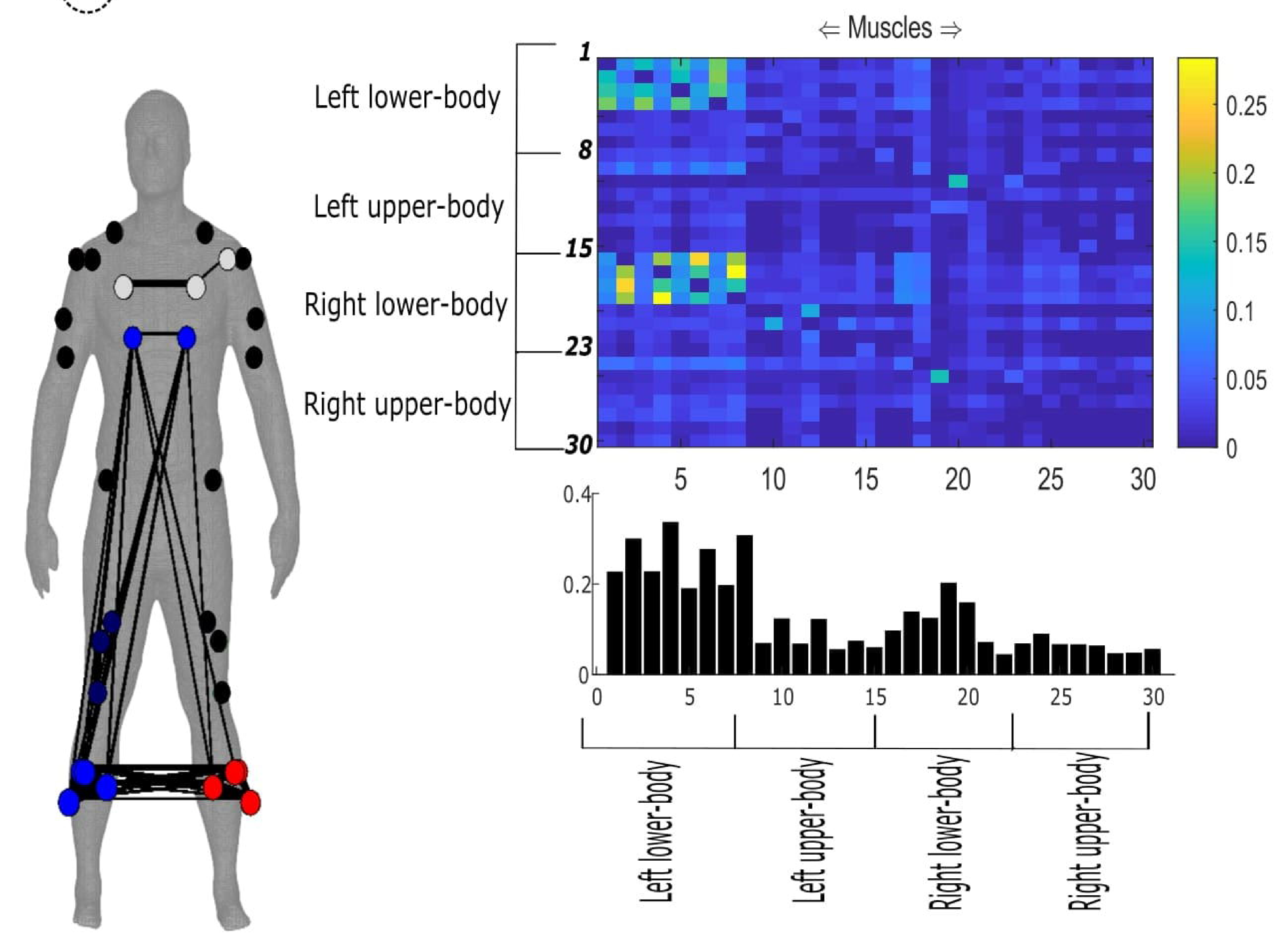

S2

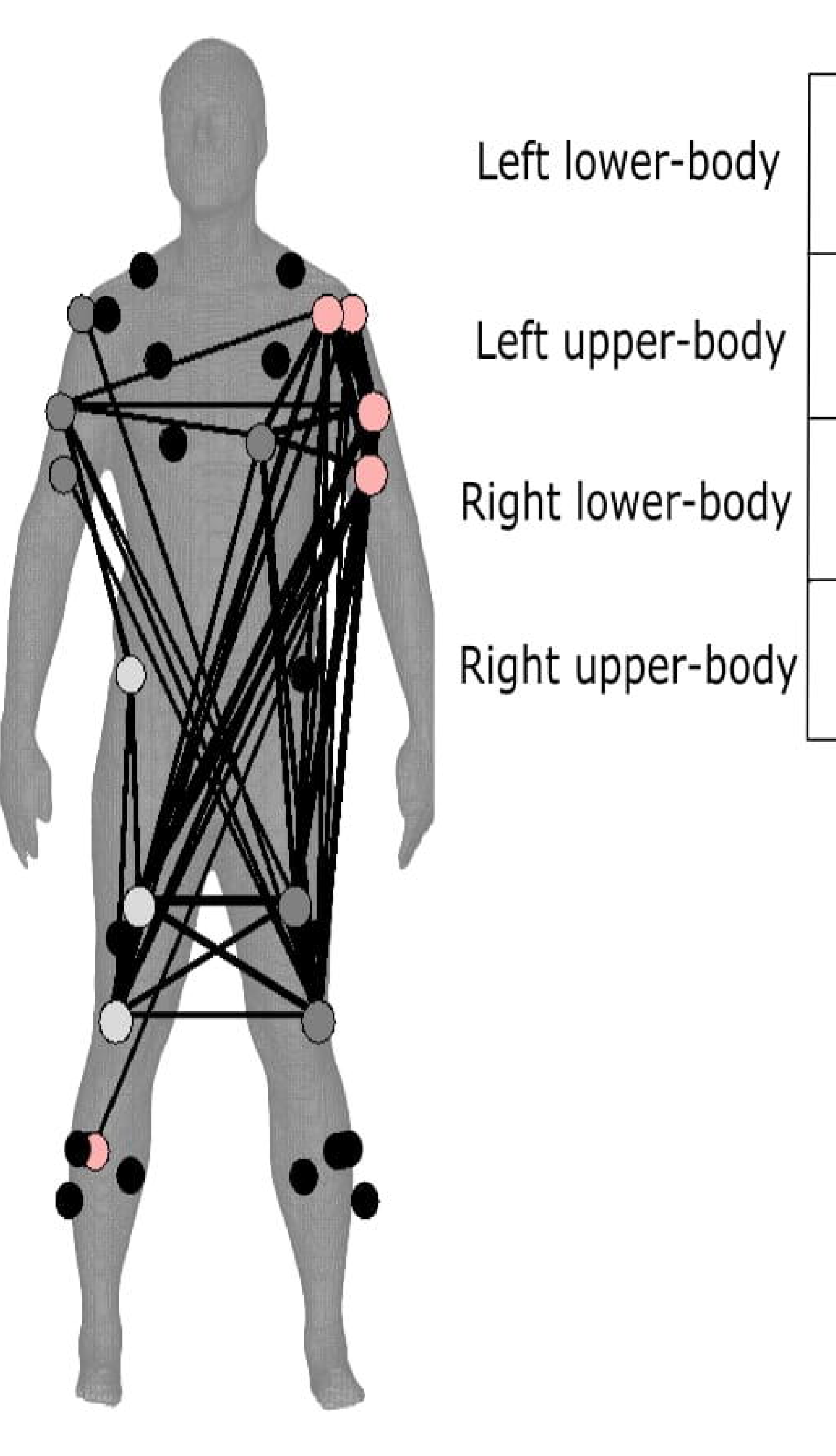

S3
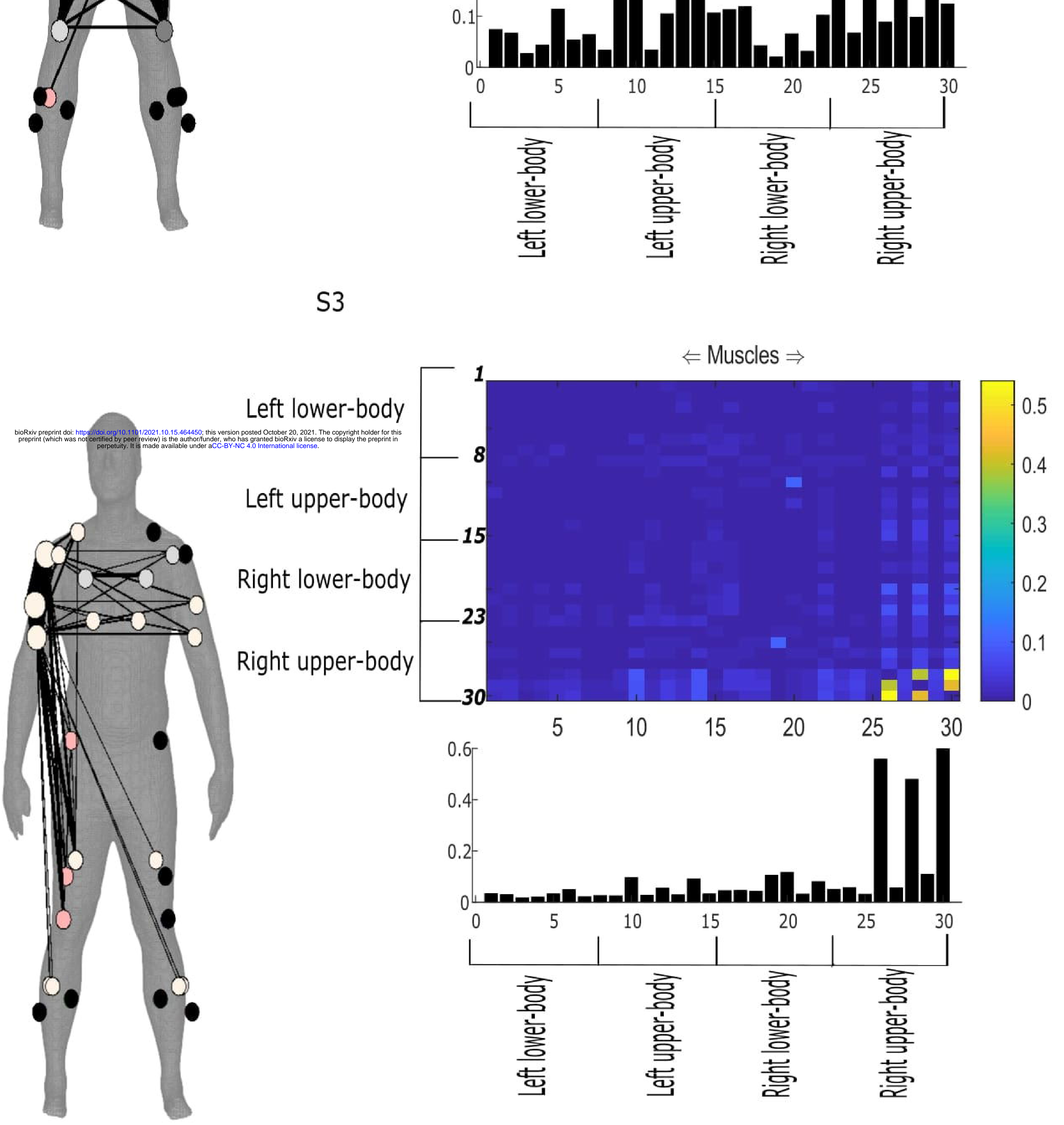
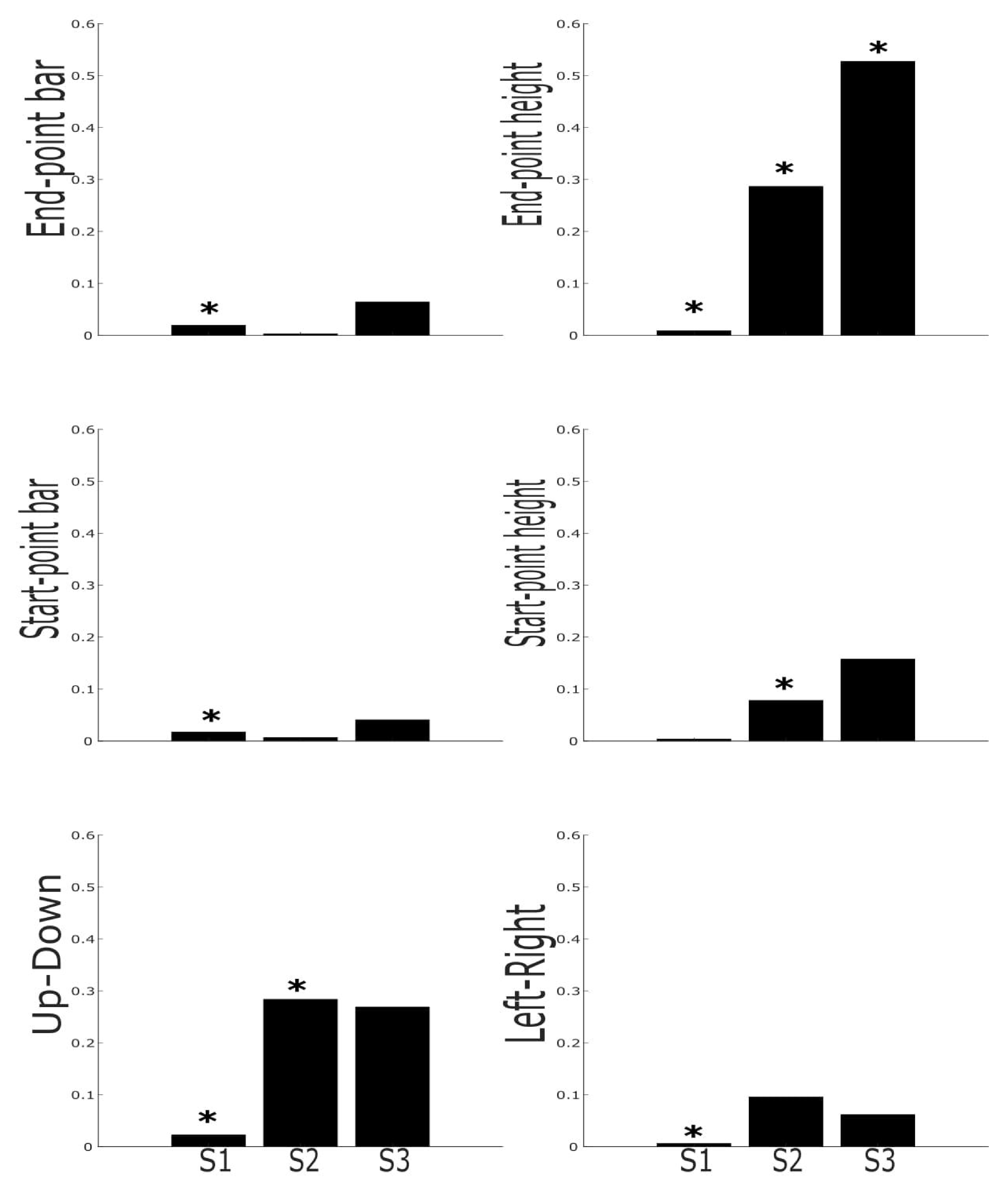

c
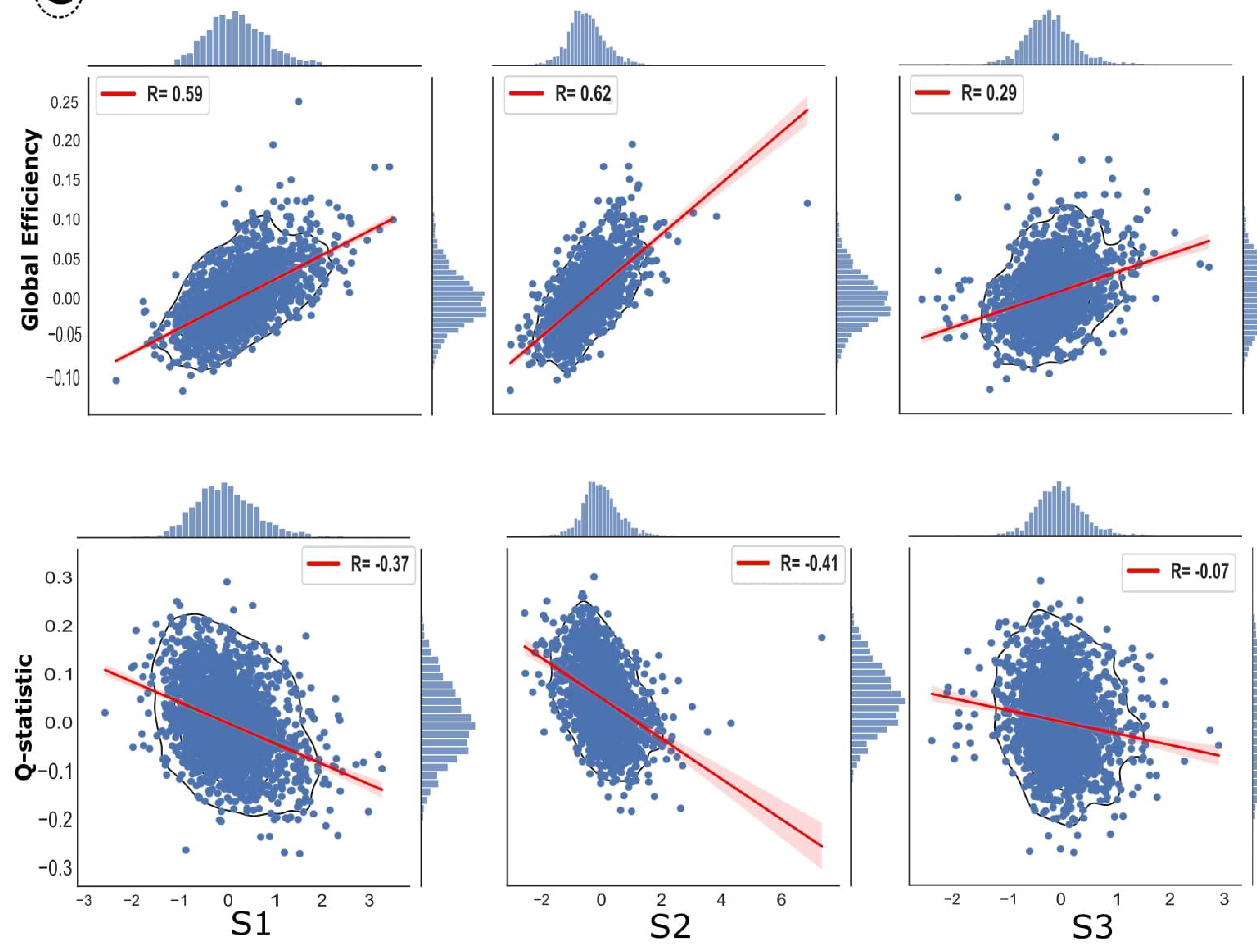


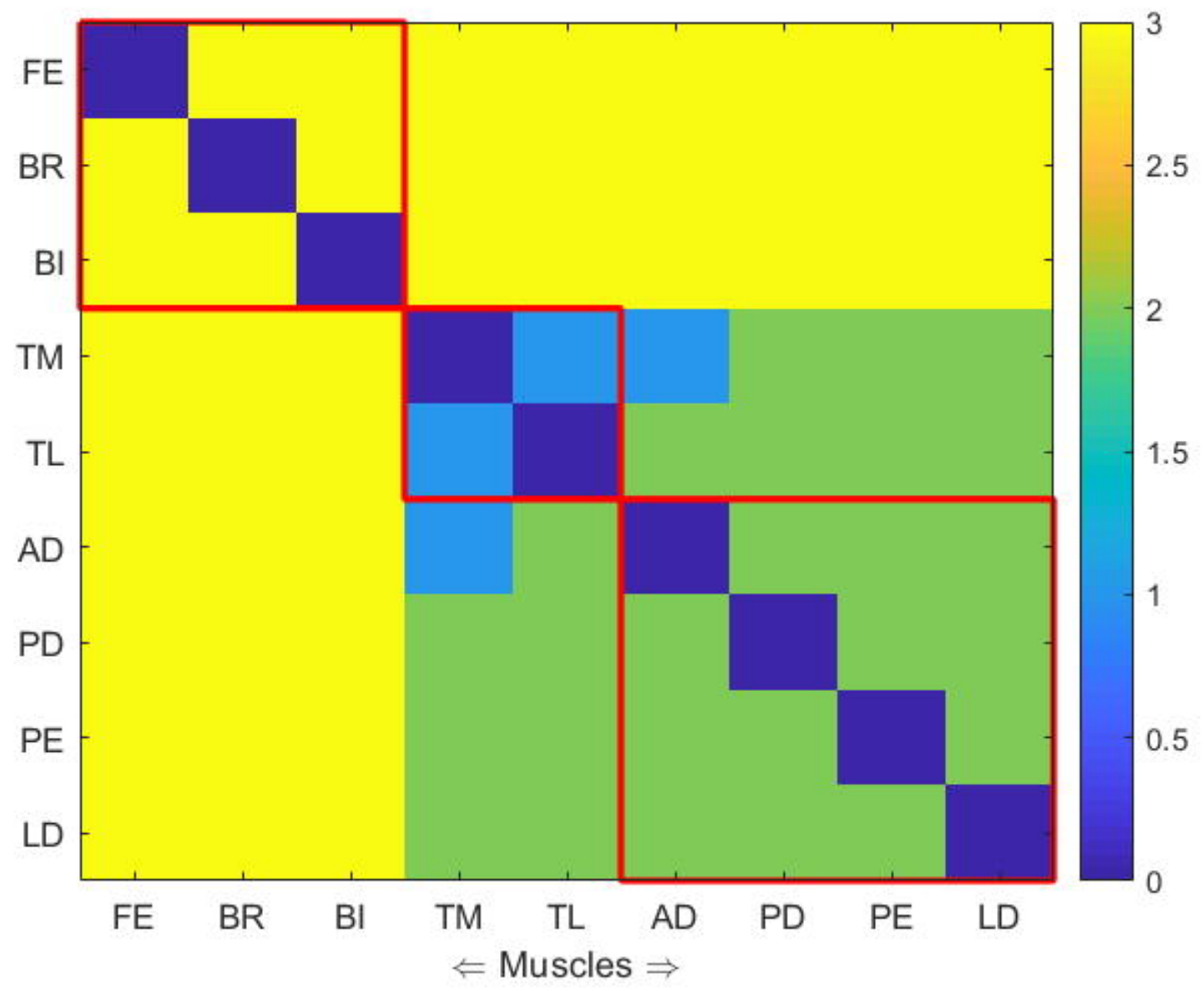




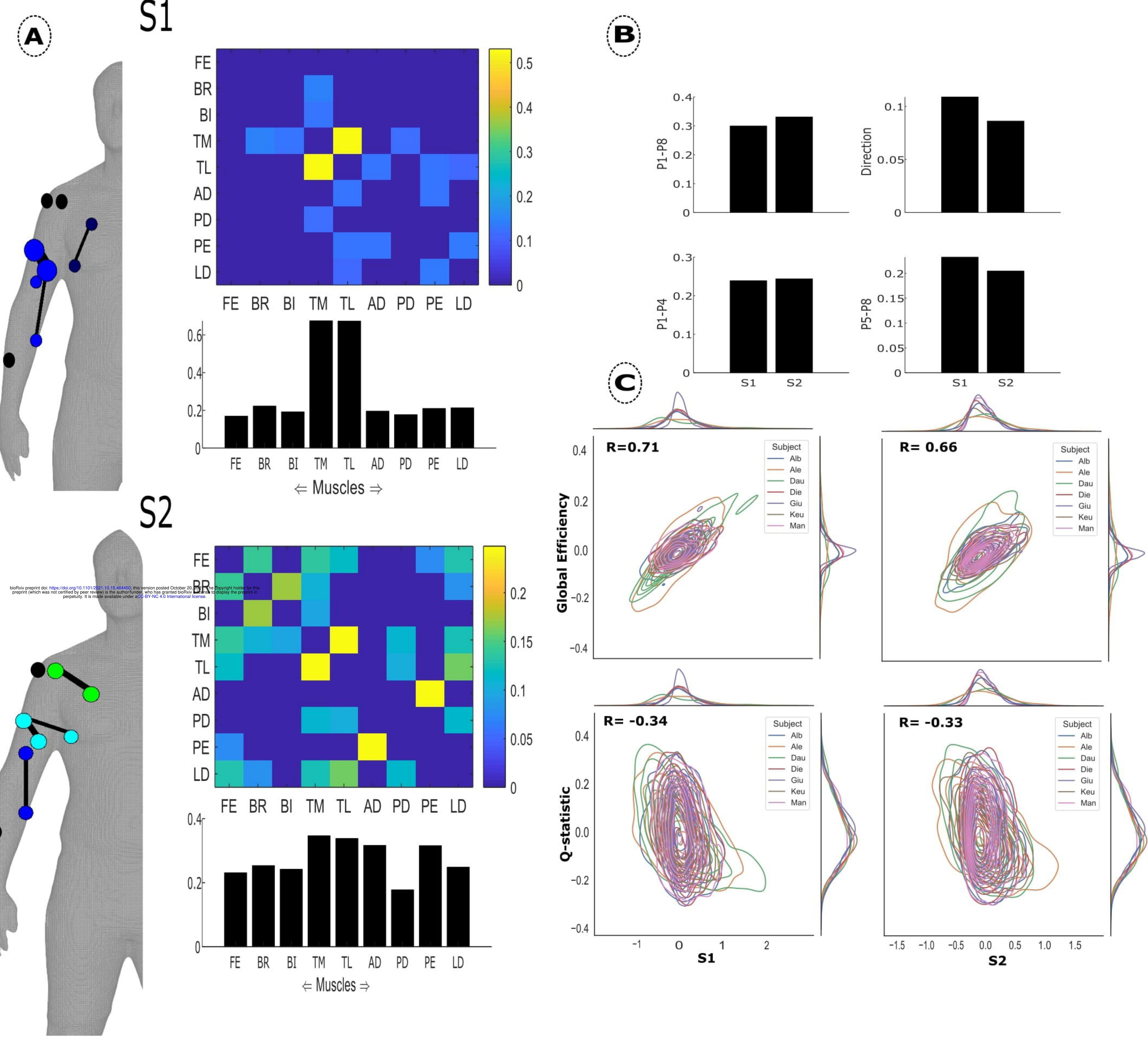


ST1

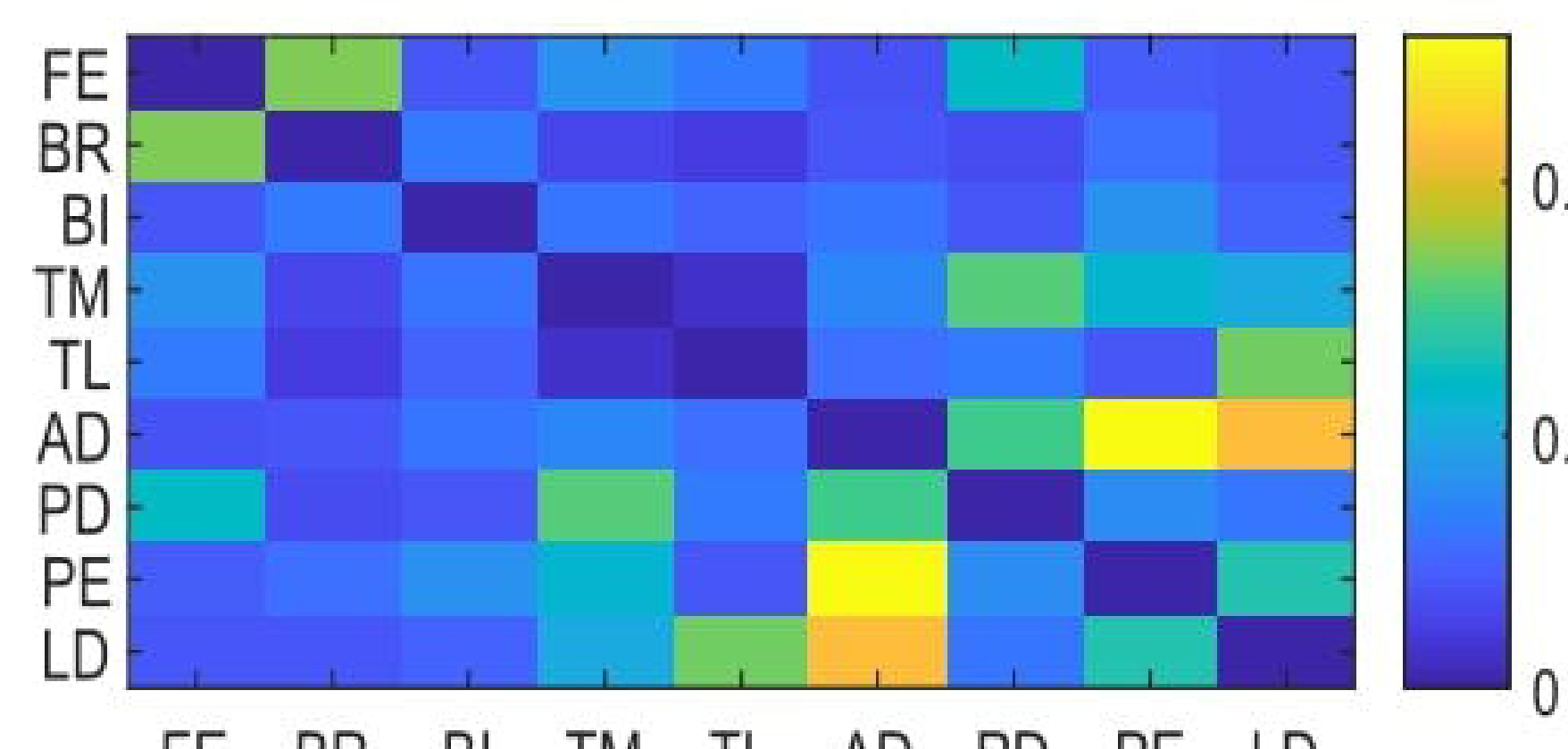

$\begin{array}{llllllllll} & F E & B R & B I & T M & T L & A D & P D & P E & L D\end{array}$

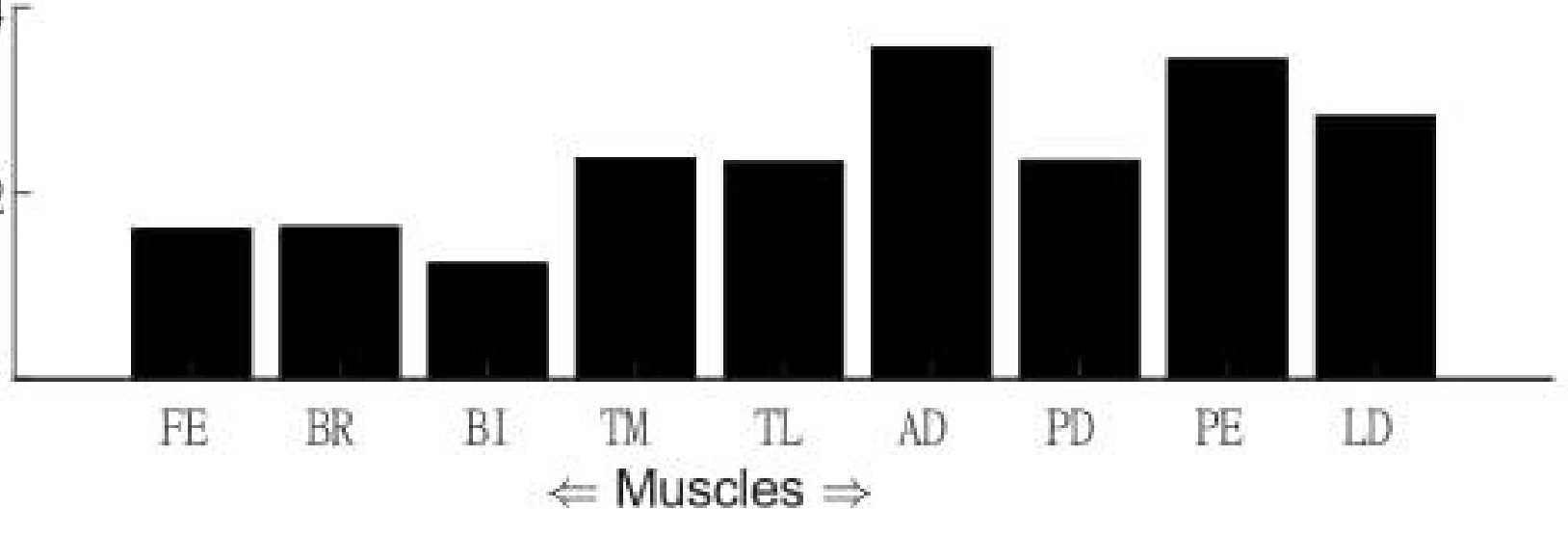

ST2

$\mathrm{F}$
$\mathrm{B}$
$\mathrm{E}$
$\mathrm{T}$
$\mathrm{T}$
$\mathrm{Al}$
$\mathrm{Pl}$
$\mathrm{P}$
$\mathrm{L}$
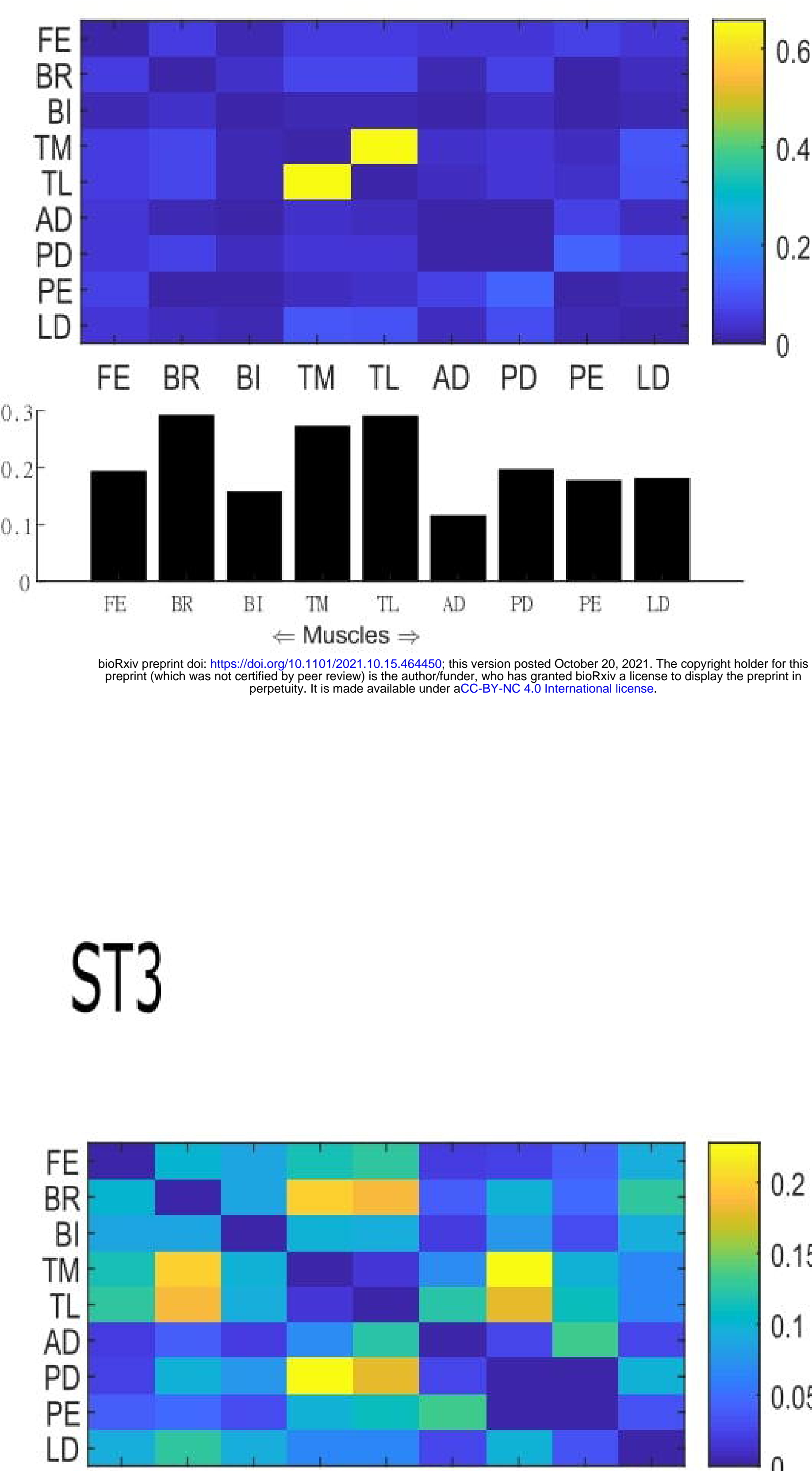

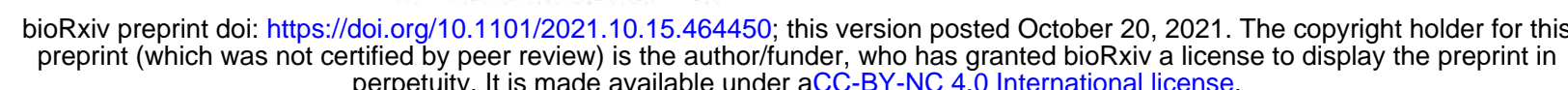

ST3

P

$$
0.6
$$$$
\begin{array}{lllllllll} 
& F E & B R & B I & T M & T L & A D & P D & P E
\end{array}
$$$$
\text { FE }{ }_{B R} \quad{ }_{B I}
$$
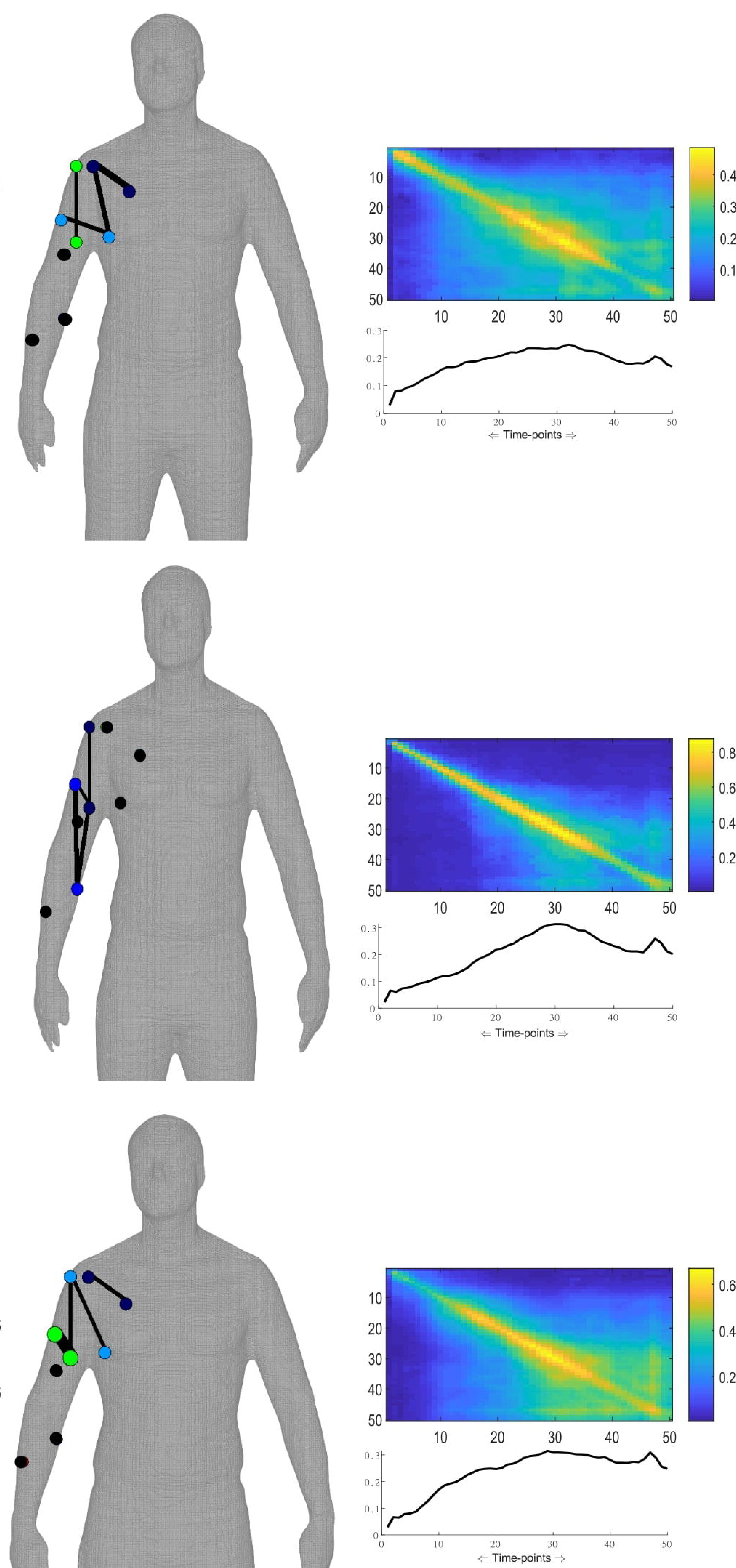
A

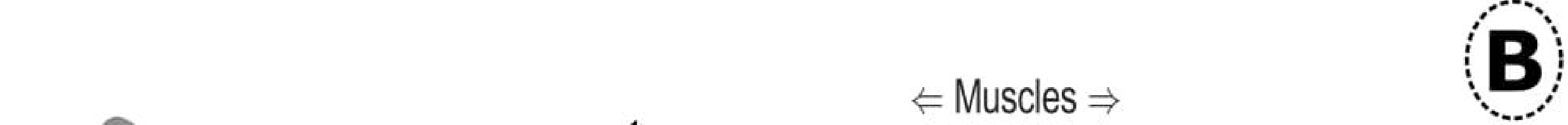

$\Leftrightarrow$ Muscles $\Rightarrow$

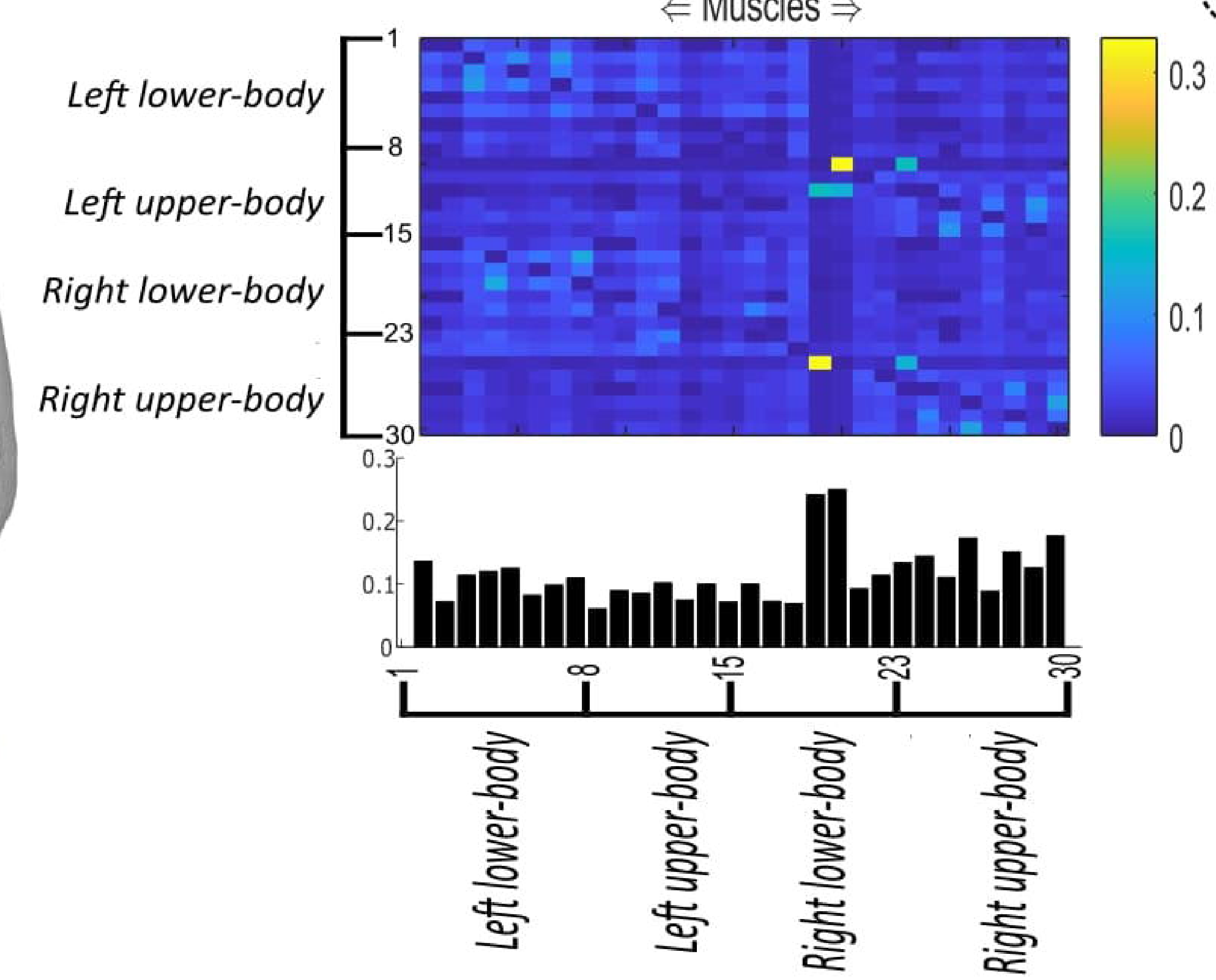

S2
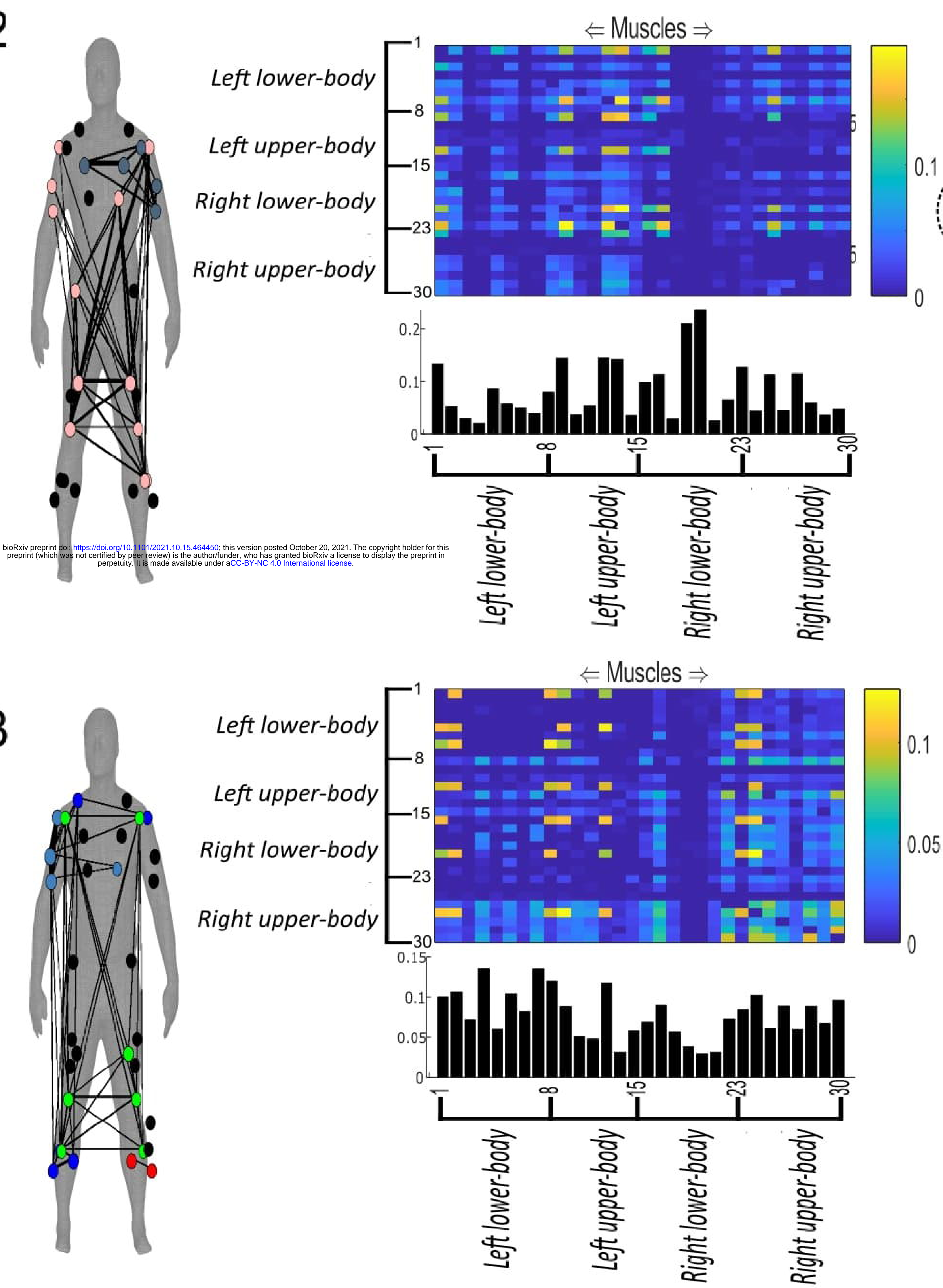

C)
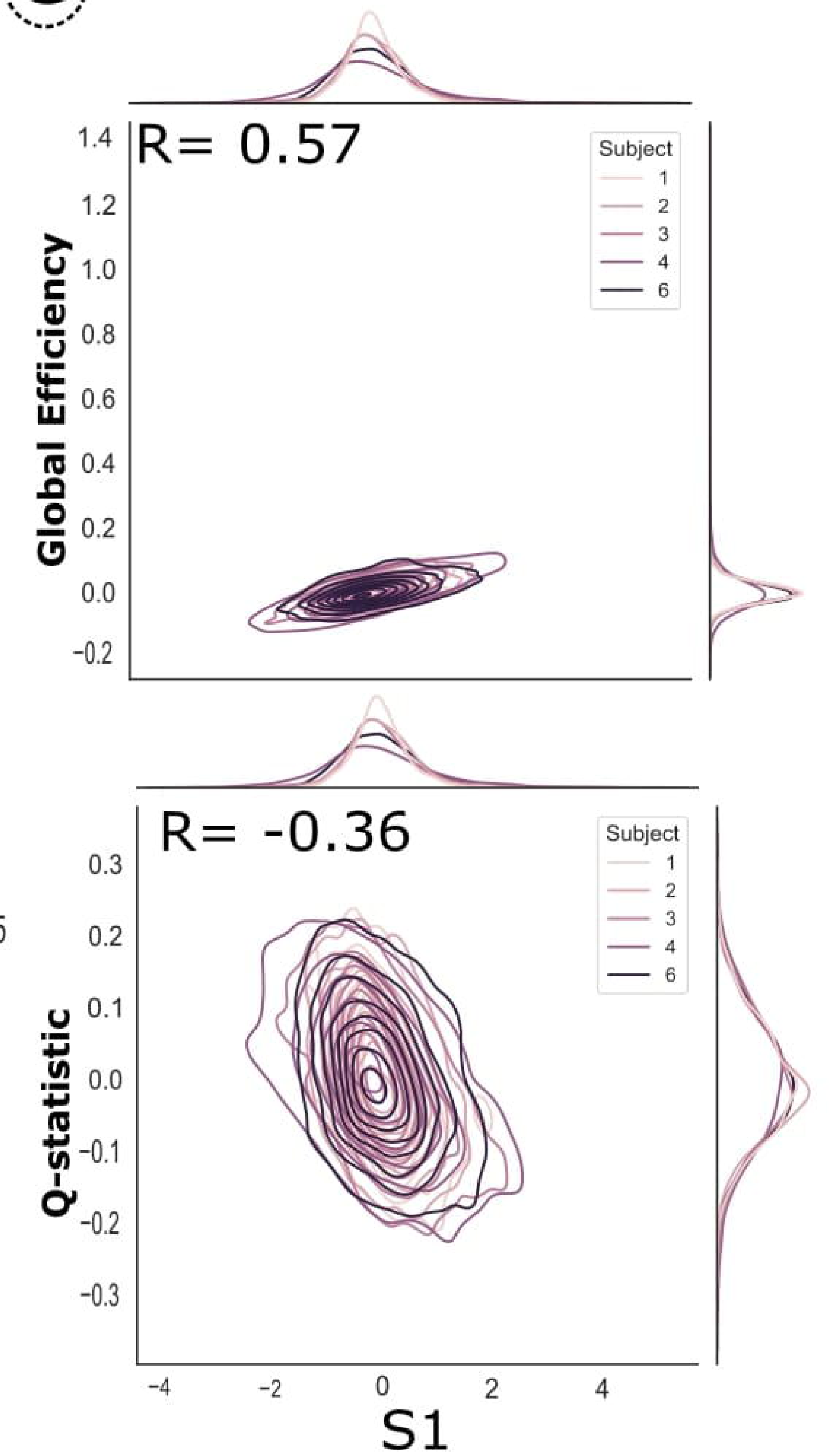
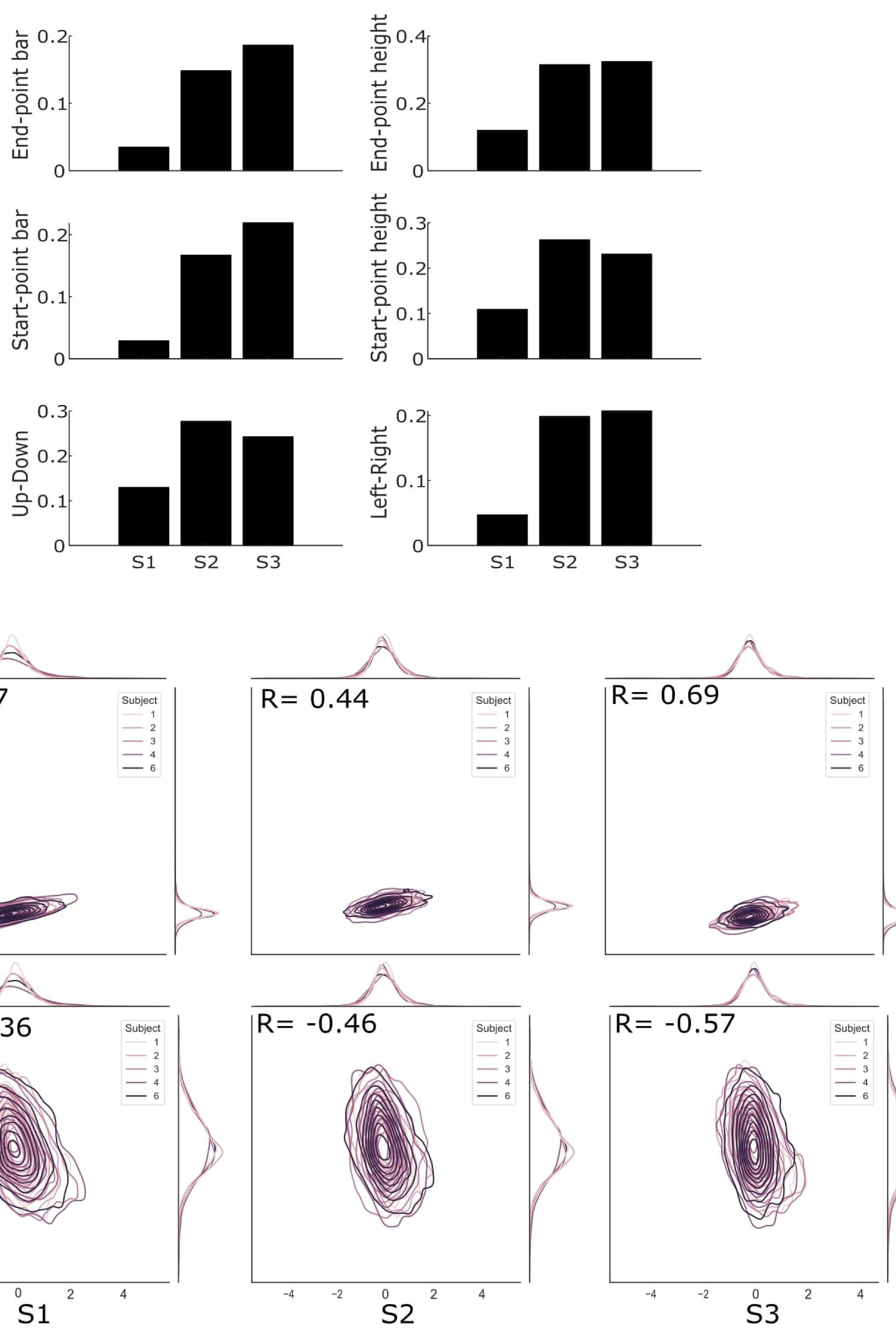


\section{T1}
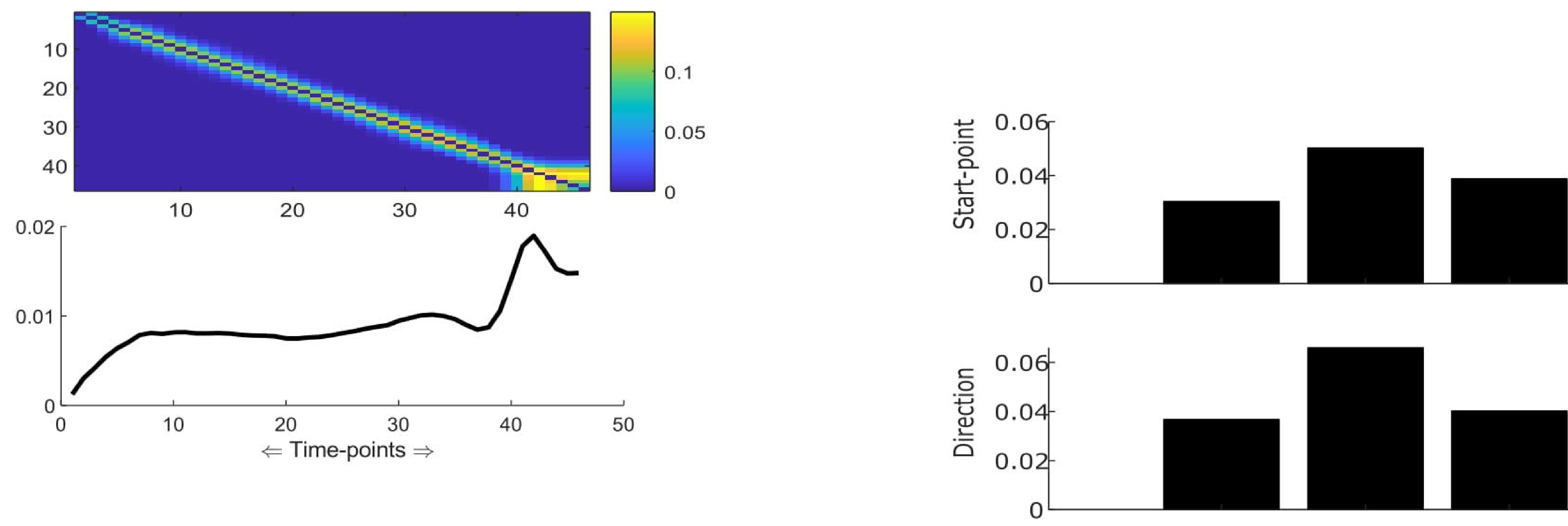

T2

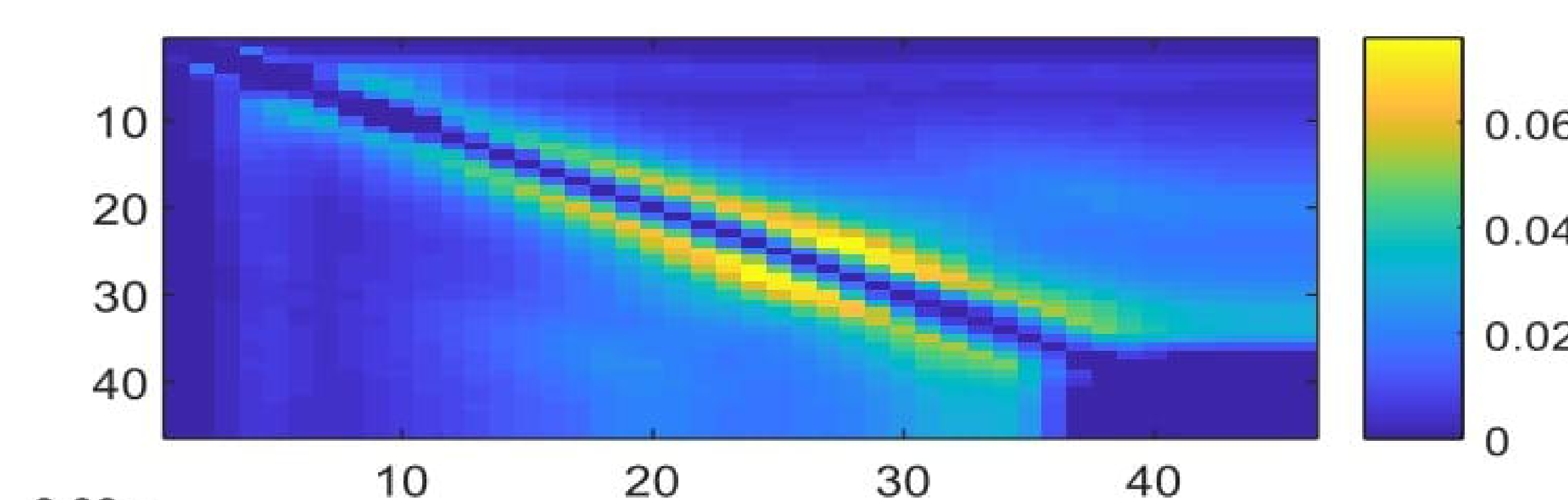

${ }^{\circ} \mathrm{C}$
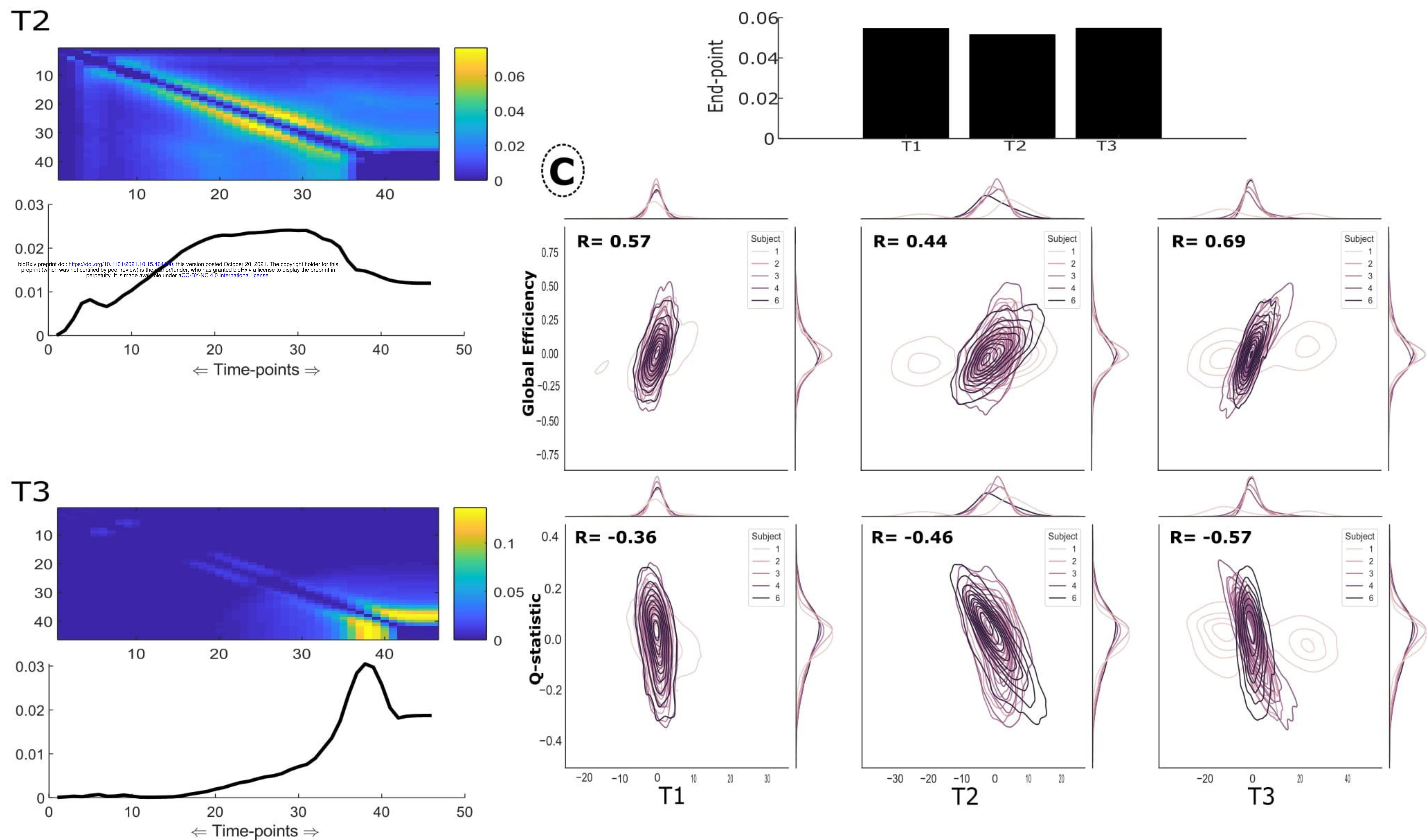
